\title{
Application of Monte Carlo algorithms to the calculation of dose and RBE for low energy brachytherapy
}

Citation for published version (APA):

White, S. (2015). Application of Monte Carlo algorithms to the calculation of dose and RBE for low energy brachytherapy. [Doctoral Thesis, Maastricht University]. Datawyse / Universitaire Pers Maastricht. https://doi.org/10.26481/dis.20151118sw

Document status and date:

Published: 01/01/2015

DOI:

10.26481/dis.20151118sw

Document Version:

Publisher's PDF, also known as Version of record

\section{Please check the document version of this publication:}

- A submitted manuscript is the version of the article upon submission and before peer-review. There can be important differences between the submitted version and the official published version of record.

People interested in the research are advised to contact the author for the final version of the publication, or visit the DOI to the publisher's website.

- The final author version and the galley proof are versions of the publication after peer review.

- The final published version features the final layout of the paper including the volume, issue and page numbers.

Link to publication

\footnotetext{
General rights rights.

- You may freely distribute the URL identifying the publication in the public portal. please follow below link for the End User Agreement:

www.umlib.nl/taverne-license

Take down policy

If you believe that this document breaches copyright please contact us at:

repository@maastrichtuniversity.nl

providing details and we will investigate your claim.
}

Copyright and moral rights for the publications made accessible in the public portal are retained by the authors and/or other copyright owners and it is a condition of accessing publications that users recognise and abide by the legal requirements associated with these

- Users may download and print one copy of any publication from the public portal for the purpose of private study or research.

- You may not further distribute the material or use it for any profit-making activity or commercial gain

If the publication is distributed under the terms of Article 25fa of the Dutch Copyright Act, indicated by the "Taverne" license above, 
Application of Monte Carlo

algorithms to the calculation

\author{
of dose and RBE for
}

low energy brachytherapy 
Copyright

Print: Datawyse - Universitaire Pers Maastricht ISBN: 9789461595010 


\section{Application of Monte Carlo algorithms to the calculation of dose and RBE for low energy brachytherapy}

Dissertation

to obtain the degree of Doctor at Maastricht University, on the authority of the Rector Magnificus, Prof.dr. L.L.G. Soete in accordance with the decision of Board of Deans, to be defended in public on Wednesday 18th November 2015 at 10:00 hours, in Maastricht

By

Shane White

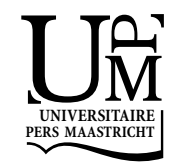




\section{Supervisors}

Prof. Dr. Frank Verhaegen

\section{Co-supervisor}

Dr. Brigitte Reniers

\section{Assessment Committee}

Prof. Dr. Philip Lambin (chairman), Maastricht University

Prof. Dr Frank-Andre Siebert Kiel

Prof. Dr. Dirk Verellen, Vrije Universiteit Brussel

Dr. Michel Oellers, Maastricht University

These studies were funded by the MAASTRO-ATRIUM Grant 
Dedicated to the love of my life, Scarlett. 



\section{Table of contents}

Chapter 1: Introduction and outline of thesis............................................

Chapter 2: Influence of trace elements in human tissue in low energy photon brachytherapy dosimetry.................................................................................21

Chapter 3: Comparison of TG-43 and TG-186 in breast irradiation using a low energy electronic brachytherapy source.................................................. 35

Chapter 4: Measurement of absorbed dose to water around an electronic brachytherapy source. Comparison of two dosimetry systems: lithium formate

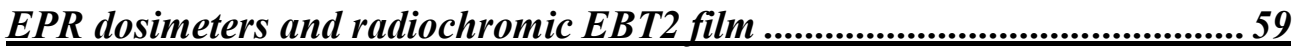

Chapter 5: A comparison of the relative biological effectiveness of low energy electronic brachytherapy sources in breast tissue: $A$ Monte Carlo studv....... 77

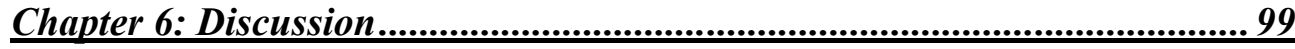

Chapter 7: Valorization addendum.............................................................. 107

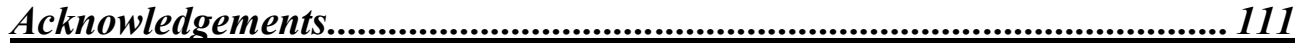



Chapter 1:

Introduction and outline of thesis 


\section{Introduction}

Brachytherapy is a form of radiotherapy that utilizes small encapsulated radiation sources placed close to or inside a target volume. As with other forms of radiotherapy, brachytherapy involves the delivery of a prescribed dose of radiation to a clinically relevant volume within the body for the purpose of killing cancerous cells. As one of the oldest forms of brachytherapy, the design of radiation sources has changed over the decades. Low energy brachytherapy, where photons with energy below $50 \mathrm{keV}$ are used to treat patients, has been a topic in a recent AAPM Radiation Therapy Task Group, TG-186(Beaulieu et al., 2012). TG186 has highlighted short-comings in the current protocol with regard to the water-based dose calculation models currently used as described in TG-43U1(Rivard et al., 2004b).

Low energy brachytherapy can utilize high dose rate (HDR) and low dose rate (LDR) sources and can be further subdivided into the mechanism of photon emission. Traditional low energy brachytherapy sources have used radionuclides such as palladium-103 and iodine-125 in the treatment of breast and prostate brachytherapy(Blasko et al., 2000; Pignol et al., 2006a; Schellhammer et al., 1977). These types of sources are typically LDR and are permanently implanted into the patient. Low energy HDR brachytherapy uses electronic brachytherapy sources (EBS) such as the Xoft Axxent(Rivard et al., 2006b) and Zeiss Intrabeam(KrausTiefenbacher et al., 2005).

\section{Current state of the art: TG-43U1}

The current dose calculation platform used in the clinical environment, AAPM TG-43U1, uses water-based models to predict the dose distribution around sources. Dose to any $2 \mathrm{D}$ point around a source can be calculated using the equation (or: expression):

$$
\dot{D}(r, \theta)=S_{K} \cdot \Lambda \cdot \frac{G_{L}(r, \theta)}{G_{L}\left(r_{0}, \theta_{0}\right)} \cdot g_{L}(r) \cdot F(r, \theta)
$$

Where $S_{k}$ is the source strength, $\Lambda$ is the dose-rate constant, $G_{L}(r, \theta)$ is the correction to account for dose-rate falloff at distance $r$ and angle $\theta$ along the sources transverse plane, due to the inverse square law compared to the reference distance $r_{0}(1 \mathrm{~cm})$ and angle $\theta_{0}(90$ degrees $). \mathrm{g}_{\mathrm{L}}(\mathrm{r})$ is the radial dose function and reflects the dose fall-off due to radiation absorption and scattering. $\mathrm{F}(\mathrm{r}, \theta)$ is the anisotropy function and corrects the change in doserate as a function of angle due to source construction. $\mathrm{F}(\mathrm{r}, \theta)$ and $\mathrm{g}_{\mathrm{L}}(\mathrm{r})$ originate from lookup tables derived for the particular brachytherapy source and are calculated using MC simulations and/or measured using experimental methods such as TLD, radiochromic film or ion chamber measurements. Dose-rates from multiple sources are calculated from the superpositions of individual sources. TG-43U1 is advantageous for its dose calculation speed and its reasonable accuracy in simple homogeneous water-equivalent geometries with single high energy sources such as Ir-192. Deviations in accuracy become more apparent at lower energies and in more complex heterogeneous setups. 


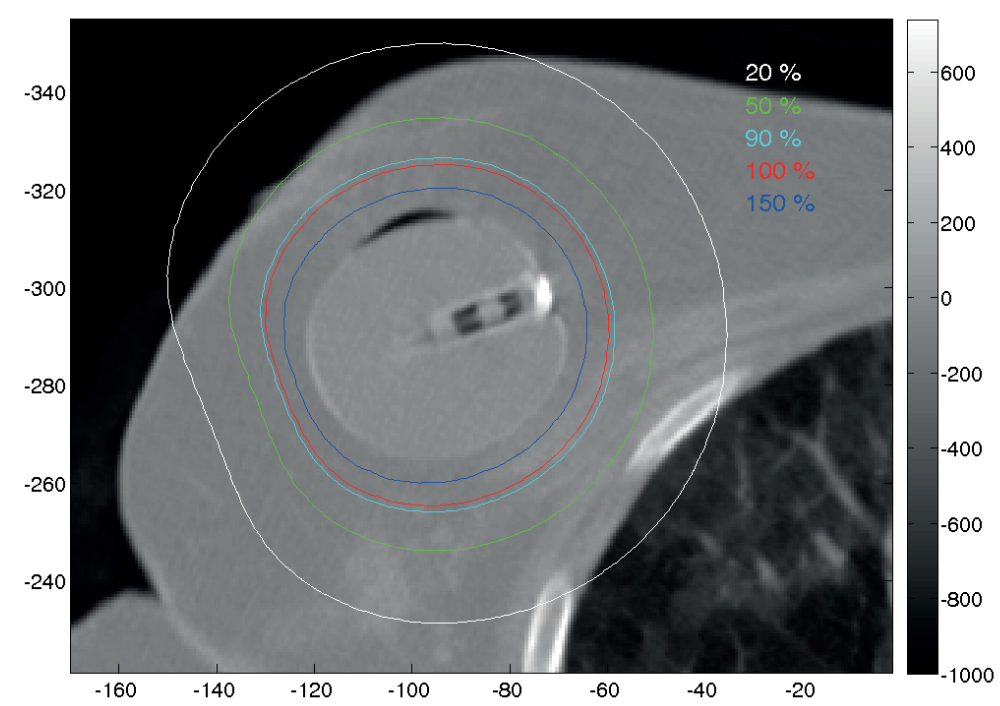

Figure 1: CT-based TG-43 treatment plan of APBI patient calculated using BrachyVision. The dose distribution (coloured lines as a percentage of prescription dose 34Gy) ignores the effect of tissue heterogeneities (shown in HU scale, absence of skin backscatter and applicator effects. The air and rib do not perturb the dose distribution

\section{Problems in brachytherapy}

\section{Tissue heterogeneity}

The use of low energy brachytherapy sources presents greater challenges in relation to dose prediction than with high energy sources. While low energy photons have a steep dose falloff due to higher attenuation, the degree of attenuation depends strongly on the tissue composition with tissue of a higher atomic number $(Z)$, such as rib or calcium-rich breast tissue, more strongly attenuating than low- $Z$ tissue like adipose. The sensitivity of low energy photons to population variations in adipose tissue has been demonstrated (Landry et al., 2010b), where varying concentration of hydrogen, oxygen and carbon results in significant differences in the dose distribution around a brachytherapy source. TG-186 recommends the use of more advanced model-based dose calculation algorithms (MBDCA) which in turn require more detailed descriptions of the patient geometry and composition. Accurate patient modeling requires realistic patient geometrical models which can be obtained using CT imaging (Afsharpour et al., 2012). As CT-imaging distinguishes tissue based on HU/ mass density, proper identification of different tissue types is required. Current treatment planning systems (TPS) for brachytherapy ignore tissue heterogeneity and patient geometry and assume the TG-43U1 approach, with all tissues assigned the composition and density of water. The patient geometry is assumed to be a sphere $15 \mathrm{~cm}$ in radius with the source at the centre. This approach is advantageous for dose calculation due to its simplicity. TG-43U1 allows for an easy comparison of dose calculations between patients. 


\section{Dose reporting}

The method of reporting absorbed dose in low energy brachytherapy is controversial (Walters et al., 2010) (Tedgren and Carlsson, 2013) due to the different nature of the dose quantities calculated. While radiation can be attenuated by any medium in a phantom, how the locally deposited dose is reported in MC is still a matter of discussion. Arguments for scoring and reporting the dose in the same medium as it was transported in (Radiation transported in medium, dose scored in medium: $\mathrm{D}_{\mathrm{m}, \mathrm{m}}$ ) are that it is the quantity inherently calculated in Monte Carlo platforms and accurately reflects the physical dose given to tissue. However an alternative method, dose to water (Radiation transported in medium, dose scored in water: $\mathrm{D}_{\mathrm{w}, \mathrm{m}}$ ) has been used extensively in TG-43 and the most clinical experience exists. In TG$43 \mathrm{U} 1$, all tissue was treated as water for photon transport and dose reporting $\left(\mathrm{D}_{\mathrm{w}, \mathrm{w}}\right)$ Arguments for the continued use of reporting $\mathrm{D}_{\mathrm{w}, \mathrm{m}}$ are that human cells, where the most clinically relevant dose is deposited, is composed mostly of water and bulk tissue composition does not make a good substitute (Liu et al., 2002). Conversion from $\mathrm{D}_{\mathrm{m}, \mathrm{m}}$ to $D_{\mathrm{w}, \mathrm{m}}$ is possible(Tedgren and Carlsson, 2013) but may introduce greater uncertainties due to inaccuracies in the conversion factors (Tedgren and Carlsson, 2013) (see Dosimetry) TG-186 recommends the continued use of TG- $43 \mathrm{U}$ dose calculation alongside $\mathrm{D}_{\mathrm{m}, \mathrm{m}}$ and $\mathrm{D}_{\mathrm{w}, \mathrm{m}}$ results.

\section{Dosimetry}

Accurate dose delivery in low energy brachytherapy also requires in-vivo dosimetry measurements, to ensure the prescribed dose has been delivered to the target, that dose to organs at risk has been minimized and to validate dose calculation algorithms particularly those using novel modeling techniques. Dosimetry measurements with low energy sources are inherently difficult and complex due to the high dose gradients and the large range of dose rates involved. Uncertainties in positioning of either the source or detector are magnified at close distances. For a linear source, the dose gradient is approximately $50 \% / \mathrm{mm}$ at $4 \mathrm{~mm}$ (Tanderup et al., 2013), rendering even the dimensions of a small detector such as a thermoluminescence detector (TLD) important as they can no longer be consider to be pointobjects. While the effect of the dose gradient decreases with distance (e.g. $9 \% / \mathrm{mm}$ at $20 \mathrm{~mm}$ ), increased distance from the source reduces the signal to noise ratio (SNR).

Radiation detectors also must accommodate the energy dependence issues encountered when using detectors more commonly used in high megavoltage sources such as ion-chambers. To account for the physical and composition differences inherent with different heterogeneous chamber designs, a series of assumptions about how each detector measures dose are made. According to the Bragg-Gray Cavity theory, a gas filled cavity, serving as an ion chamber/detector, embedded in a medium can accurately measure the absorbed dose, provided the cavity is sufficiently small so that it does not alter the passage or fluence of electrons throughout the medium thereby allowing for charged-particle equilibrium (CPE). As the range of secondary electrons varies as a function of incident photon energy, the choice of cavity size required to achieve CPE in low energy brachytherapy sources can reach an impractically small nanometers. Photon- based detector using on mass-attenuation absorption co-efficents (large cavities) are more energy dependant as the photo-electric effect which dominates more at low energies(Tanderup et al., 2013). Though ideal detectors should be 
energy independent, the energy dependence of the detectors results in an over-response for low energies. . Low energy sources may also experience significant spectral changes with increasing distance from the source due to spectral hardening, where lower energy photons are filtered out of the photon spectrum more than higher energy photons. This creates a major challenge for energy dependent detectors. Detector types for brachytherapy dosimetry include MOSFET arrays (Bloemen-van Gurp et al., 2009), TLDs (Perera et al., 2005), Alanine detectors(De Angelis et al., 1999), optically stimulated luminescence dosimeters(Sharma and Jursinic, 2013), Radiochromic film(Uniyal et al., 2012) and plastic scintillation detectors(Kirov et al., 1999; Thomadsen et al., 2005). Each detector type has advantages and disadvantages in relation to its size, ease of use, availability, readout time, calibration, SNR, volume averaging, energy response, dose perturbations and biocompatibility. Readout time is an important factor in the use of detectors in permanent or single fraction brachytherapy as detectors requiring a long readout time, such as gafchromic film, TLDs and alanine, would not allow corrections to a treatment plan, should an under/over-dosing of a critical organ be measured.

\section{Radiobiological effectiveness}

The relative biological effectiveness (RBE) is defined as the ratio of the doses required by two radiation sources to cause the same level of biological effect. Sources with a higher RBE produce more cell damage per unit radiation. RBE is determined by the dose rate, total dose, level of fractionation and by a source's beam quality/spectrum. Currently, the International Commission on Radiation Protection have assigned a radiation weighting factor of unity for all photon emitting sources (ICRP, 2007), equating the biological effectiveness of high and low energy photon sources. Softer x-rays have been shown (Nikjoo and Lindborg, 2010) to increase the number of DNA strand breaks in cells, which is indicative of a greater RBE for low energy sources. As the spectrum of low energy sources may soften/harden with distance, the beam quality/RBE within the patient may vary throughout. Variations in RBE due to changes in beam quality are apparent in hadron therapy where the linear energy transfer (LET) of the beam increases with decreasing particle energy. LET mapping in particle therapy has been previously explored as a method of calculating the RBE-weighted dose maps (Grassberger et al., 2011). Similar maps in low energy brachytherapy have not been generated.

\section{Backscatter}

For sources placed close to the surface of skin, the lack of radiation backscatter is not accounted for in TG-43U calculations. This can result in over-predicting the dose to critical organs and tumour volumes (ref) as many photons that are lost to the surroundings due to lack of attenuation are instead predicted to deposit dose locally. Lack of backscatter may also occur in regions close to low density regions in the body such as lungs and to a lesser extent, the rectum for prostate brachytherapy. The degree of under-dosage due to lack of backscatter material in HDR brachytherapy has been found to depend strongly (8-13\% for depth from $1.5 \mathrm{~cm}$ to $0.5 \mathrm{~cm}$ ) on the relative position of the prescription point with larger under-dosage at greater prescription depths(Raina et al., 2005).. 


\section{Interseed attenuation}

As well as tissue heterogeneities and backscatter, the impact of the source itself is unaccounted for in current TPS dose calculations algorithms. In the case of permanent brachytherapy for the prostate and breast, the sources involved in dose delivery, with a few exceptions(Khan et al., 2008), are encapsulated in high-Z materials such as stainless steel or titanium for biocompatibility. The shape, composition and construction of the outer walls of these sources is accounted for in the anisotropic and radial dose function data used in the TPS. In the case of multi-seed implants, a shielding effect called inter-seed attenuation (ISA) occurs where one seed will attenuate radiation from another. As TG-43U1 calculates the dose-rate for multiple sources via the superposition of individual sources, the impact of the ISA is not calculated. Dose reduction due to ISA has been well-documented (Afsharpour et al., 2008; Afsharpour et al., 2010; Carrier et al., 2006; Chibani et al., 2005; Mason et al., 2013). Post-implant dose calculations of LDR prostate brachytherapy patients using TG$43 \mathrm{U} 1$ and $\mathrm{MC}$ simulations have revealed a $5.8 \%$ to $12.8 \%$ decrease in $\mathrm{D}_{90}$ when ISA and tissue compositions are taken into account (Carrier et al., 2006). The $\mathrm{D}_{90}$ impact of spectral and design variations of six different seed types was also explored using MC simulations where variations in the composition of the seed encapsulation influenced the degree of $\mathrm{D}_{90}$ reduction by between $4.8 \%$ to $1.5 \%$. $\mathrm{D}_{90}$ reduction was the least for the polymer encapsulated Pd-103 OptiSeed (International Brachytherapy).

\section{Applicator attenuation}

HDR treatments typically involve a single source placed into multiple dwell positions until the desired dose distribution is achieved. As such there is no inter-source attenuation. HDR brachytherapy may require the use of applicators to ensure accurate positioning inside the patient or to avoid high dose gradients close to the source. Applicators used in high and low energy brachytherapy are ignored in TG-43U1. The neglect of applicator attenuation in high$\mathrm{Z}$ uterine applicators has been shown to lead to appreciable over-dosing errors (3.5\%) along the transverse plane for an Ir-192 source (Ye et al., 2004). Similar MC studies have shown similar applicator effects on the dose to target volume in endorectal patients with applicator shields reducing the target volume dose from $97 \%$ to $94 \%$ in one study(Poon et al., 2008b) and while tissue heterogeneities have not been shown to significantly alter predicted dose compared to TG-43U1 protocols in gynecological brachytherapy, the exclusion of air and applicator volumes has been noted as an important issue in another study(Desbiens et al., 2013).

Applicators in low energy brachytherapy sources are ultized for skin (Rong and Welsh, 2010), rectal (Guo et al., 2012; Richardson et al., 2012) and breast (Dickler, 2009; Vaidya et al., 2001). Of particular interest for this thesis are applicators used in accelerated partial breast irradiation (APBI). The Xoft ${ }^{\circledR}$ Axxent is an EBS used in APBI in a manner similar to MammoSite, a Ir-192 balloon catheter-based treatment used in breast irradiation (Edmundson et al., 2002), with exception to the type of source used. The Axxent inflatable balloon applicator, designed to anchor itself inside the breast cavity during irradiation, contains high$\mathrm{Z}$ elements which eases the delineation between the applicator surface and surrounding tissue during CT imaging for treatment planning. The impact of the Axxent balloon applicator on the dose distribution has been studied (Segala et al., 2011). An applicator may be modeling 
based on the outline visible during imaging but shortcomings in the modeling of applicators using CT-based voxelized descriptions have been highlighted (Fonseca et al., 2014).

Table 1: classifying the significance of including dose modeling factors into dose calculation algorithms compared to TG-43 dose calculation

\begin{tabular}{|l|l|l|l|l|}
\hline \multicolumn{1}{|c|}{ Source } & Pd-103 & I -125 & Ir-192 & EBS \\
\hline Significant? & Yes & Yes & Bone/lung only & Yes \\
\hline Interseed attenuation & $\begin{array}{l}\text { Yes } \\
\text { (minor in polymer seeds })\end{array}$ & Yes & - & - \\
\hline Applicator attenuation & - & - & $\begin{array}{l}\text { Yes (Metal applicators) } \\
\text { No (plastic applicators) }\end{array}$ & $\begin{array}{l}\text { Yes (Axxent) } \\
\text { No (Intrabeam) }\end{array}$ \\
\hline Backscatter & Yes & Yes & Yes & Yes \\
\hline $\begin{array}{l}\text { Dose reporting } \\
\left(\mathrm{D}_{\mathrm{m}, \mathrm{m}}, \mathrm{D}_{\mathrm{w}, \mathrm{w}} \text { or } \mathrm{D}_{\mathrm{w}, \mathrm{m})}\right.\end{array}$ & Yes & Yes & Bone/Lung dependant & Yes \\
\hline
\end{tabular}

\section{Monte Carlo dose calculation}

The TG-186 recommends the continued use of TG-43-based dosimetry alongside modelbased dose calculation algorithms. Monte-Carlo (MC) based simulations have been recommended as one suitable alternative to the TG-43 formulation. MC dose calculation allows the simulation of photon and electron transport in realistic patient geometries. Patient information such as CT or MRI images, derived from pre-treatment/diagnostic scans can be used to construct patient geometries suitable for Monte Carlo dose calculation with delineated organs. . MC simulation requires considerable time (minutes to hours depending on the number of voxels used and required accuracy (Sarrut and Guigues, 2008)) for calculation precluding it from real-time use in clinical environments, but is considered the gold-standard in dose calculation(Beaulieu et al., 2012) . 


\section{Objectives and outline of thesis}

\section{Influence of trace elements in human tissue in low-energy photon brachytherapy dosimetry}

The impact on radiation dose of population variations in $\mathrm{H}, \mathrm{C}, \mathrm{N}$ and $\mathrm{O}$ percentage mass in breast tissue has been established for low energy brachytherapy sources (Landry et al., 2010b). It has been shown (Carvalho et al., 2007b) that the concentration of trace elements $(\mathrm{Z}>8)$ in prostate and breast tissue are higher in cancerous tissue compared to healthy tissue. Current knowledge about the composition of tissue is derived from healthy human populations measured in studies widely used in ICRU reports(International Commission on Radiation Units and Measurements, 1992) (White, 1986). In addition, trace element concentration has not been measured with a high precision (rounded to 1 significant digit) with many low concentration of physiologically important elements such as iron and copper not measured at all. Given the larger photo-electric cross-section for trace elements (strongly dependant on photon energy and atomic number), it was investigated in this chapter whether the variations in the concentration of traces elements could bring a non-negligible change in a dose distribution in commonly treated tissues around a low energy brachytherapy source.

\section{Comparison of TG-43 and TG-186 in breast irradiation using a low energy electronic brachytherapy source}

In chapter 3, the recommendations described in TG-186 were applied to a series of clinical cases. 7 APBI patient cases, treated with the Xoft Axxent, underwent dose re-calculation for a more realistic model of the patient using TPS CT images. Using a Monte Carlo calculation platform, Geant4(Agostinelli et al., 2003b), the dose distribution was determined for each patient, taking into account applicator attenuation, tissue heterogeneity and backscatter correction, as recommended by TG-186. Tissue segmentation was used to distinguish breast voxels into separate tissues, given the complex gland structure and the Axxent balloon applicator was modeled using a novel mesh-based technique(Fonseca et al., 2014). Variability in breast size allowed the impact of lack of backscatter to be explored compared to all-water models. A comparison was made between dose to the planning treatment volume (PTV) and skin organ at risk (OAR) calculated with the older TG-43 and the newer TG-186.

\section{Measurement of absorbed dose to water around an electronic brachytherapy source. Comparison of two dosimetry systems: lithium formate EPR dosimeters and radiochromic EBT2 film}

Accurate dosimetry is a very important factor in the quality assurance of radiotherapy treatments. As well as providing MC experimental validation, the absence of MBDCA-based dose calculation in some clinical treatments renders dosimeters provide the only dose verification method. This is particularly true in single fraction intra-operative radiotherapy 
(IORT) where imaging or re-planning is neither available nor practical. In addition to the high dose gradients, inherent energy dependency, detector size and positioning uncertainties, the intrinsic efficiency (signal per absorbed dose) of a dosimeter may influence measurement accuracy. The accuracy of two dosimetry systems, gafchromic film (EBT2) and new EPRbased lithium formate, was explored for the Axxent $50 \mathrm{kV}$ source in chapter 4.

\section{A comparison of the relative biological effectiveness of low energy brachytherapy source in breast tissue}

In addition to tissue and applicator considerations in TG-186, the potential variation of RBE due to spectral and tissue changes may also impact dose treatment accuracy. Radiation quality influences the number of DNA strand breakages caused by radiation at a cellular level. These breakages are responsible for cell inactivation and cell death. It has been shown (Nikjoo et al., 2002)that decreases in photon energy result in increased RBE. The extent to which this is true for EBS is unexplored.

Chapter 5 explores the RBE variations between two low energy EBS, the Xoft Axxent and the Zeiss Intrabeam, which output different spectra due to their respective design. Variations due to attenuation from each source's respective applicator as well as the changes in RBE in tissues at different distances away from the source were explored using DNA damage simulators.

\section{References}

Afsharpour H, D'Amours M, Cote B, Carrier J F, Verhaegen F and Beaulieu L 2008 A Monte Carlo study on the effect of seed design on the interseed attenuation in permanent prostate implants Med Phys 35 3671-81

Afsharpour H, Landry G, D'Amours M, Enger S, Reniers B, Poon E, Carrier J F, Verhaegen F and Beaulieu L 2012 ALGEBRA: ALgorithm for the heterogeneous dosimetry based on GEANT4 for BRAchytherapy Phys Med Biol 57 3273-80

Afsharpour H, Pignol J P, Keller B, Carrier J F, Reniers B, Verhaegen F and Beaulieu L 2010 Influence of breast composition and interseed attenuation in dose calculations for post-implant assessment of permanent breast 103Pd seed implant Phys Med Biol 55 4547-61

Agostinelli S, et al. 2003 Geant4: a simulation toolkit Nuclear Instruments and Methods in Physics Research Section A: Accelerators, Spectrometers, Detectors and Associated Equipment 506 250-303

Beaulieu L, et al. 2012 Report of the Task Group 186 on model-based dose calculation methods in brachytherapy beyond the TG-43 formalism: current status and recommendations for clinical implementation Med Phys 39 6208-36

Blasko J C, Grimm P D, Sylvester J E, Badiozamani K R, Hoak D and Cavanagh W 2000 Palladium-103 brachytherapy for prostate carcinoma Int J Radiat Oncol Biol Phys 46 839-50

Bloemen-van Gurp E J, Haanstra B K, Murrer L H, van Gils F C, Dekker A L, Mijnheer B J and Lambin P 2009 In vivo dosimetry with a linear MOSFET array to evaluate the urethra dose during permanent implant brachytherapy using iodine-125 Int J Radiat Oncol Biol Phys 75 1266-72

Carrier J F, Beaulieu L, Therriault-Proulx F and Roy R 2006 Impact of interseed attenuation and tissue composition for permanent prostate implants Med Phys 33 595-604

Carvalho M L, Magalh Ã£es T, Becker M and von Bohlen A 2007 Trace elements in human cancerous and healthy tissues: A comparative study by EDXRF, TXRF, synchrotron radiation and PIXE 9th Rio Symposium on Atomic Spectrometry 62 1004-11

Chibani O, Williamson J F and Todor D 2005 Dosimetric effects of seed anisotropy and interseed attenuation for 103Pd and 125I prostate implants Med Phys 32 2557-66

De Angelis C, Onori S, Petetti E, Piermattei A and Azario L 1999 Alanine/EPR dosimetry in brachytherapy Phys Med Biol 44 1181-91 
Desbiens M, D'Amours M, Afsharpour H, Verhaegen F, Lavallee M C, Thibault I, Vigneault E and Beaulieu L 2013 Monte Carlo dosimetry of high dose rate gynecologic interstitial brachytherapy Radiother Oncol $109425-9$

Dickler A 2009 Xoft Axxent electronic brachytherapy: a new device for delivering brachytherapy to the breast Nat Clin Pract Oncol 6 138-42

Edmundson G K, Vicini F A, Chen P Y, Mitchell C and Martinez A A 2002 Dosimetric characteristics of the MammoSite RTS, a new breast brachytherapy applicator Int J Radiat Oncol Biol Phys 52 1132-9

Fonseca G P, Landry G, White S, D'Amours M, Yoriyaz H, Beaulieu L, Reniers B and Verhaegen F 2014 The use of tetrahedral mesh geometries in Monte Carlo simulation of applicator based brachytherapy dose distributions Phys Med Biol 59 5921-35

Grassberger C, Trofimov A, Lomax A and Paganetti H 2011 Variations in linear energy transfer within clinical proton therapy fields and the potential for biological treatment planning Int J Radiat Oncol Biol Phys 80 1559-66

Guo S, Reddy C A, Kolar M, Woody N, Mahadevan A, Deibel F C, Dietz D W, Remzi F H and Suh J H 2012 Intraoperative radiation therapy with the photon radiosurgery system in locally advanced and recurrent rectal cancer: retrospective review of the Cleveland clinic experience Radiat Oncol 7110

ICRP 2007 The 2007 Recommendations of the International Commission on Radiological Protection. ICRP publication 103 Ann ICRP 37 1-332

International Commission on Radiation Units and Measurements 1992 Photon, Electron, Proton and Neutron Interaction Data for Body Tissues

Khan S, Chen Z J and Nath R 2008 Photon energy spectrum emitted by a novel polymer-encapsulated 103Pd source and its effect on the dose rate constant Med Phys 35 1403-6

Kirov A S, Hurlbut C, Dempsey J F, Shrinivas S B, Epstein J W, Binns W R, Dowkontt P F and Williamson J F 1999 Towards two-dimensional brachytherapy dosimetry using plastic scintillator: new highly efficient water equivalent plastic scintillator materials Med Phys 26 1515-23

Kraus-Tiefenbacher U, Scheda A, Steil V, Hermann B, Kehrer T, Bauer L, Melchert F and Wenz F 2005 Intraoperative radiotherapy (IORT) for breast cancer using the Intrabeam system Tumori 91 339-45

Landry G, Reniers B, Murrer L, Lutgens L, Gurp E B, Pignol J P, Keller B, Beaulieu L and Verhaegen F 2010 Sensitivity of low energy brachytherapy Monte Carlo dose calculations to uncertainties in human tissue composition Med Phys 37 5188-98

Liu H H, Keall P and Hendee W R 2002 Dm rather than Dw should be used in Monte Carlo treatment planning Medical Physics 29 922-24

Mason J, Al-Qaisieh B, Bownes P, Henry A and Thwaites D 2013 Monte Carlo investigation of I-125 interseed attenuation for standard and thinner seeds in prostate brachytherapy with phantom validation using a MOSFET Med Phys $\mathbf{4 0} 031717$

Nikjoo H, Bolton C E, Watanabe R, Terrissol M, O'Neill P and Goodhead D T 2002 Modelling of DNA damage induced by energetic electrons ( $100 \mathrm{eV}$ to $100 \mathrm{keV}$ ) Radiat Prot Dosimetry 99 77-80

Nikjoo H and Lindborg L 2010 RBE of low energy electrons and photons Phys Med Biol 55 R65-109

Perera F, Chisela F, Stitt L, Engel J and Venkatesan V 2005 TLD skin dose measurements and acute and late effects after lumpectomy and high-dose-rate brachytherapy only for early breast cancer Int $J$ Radiat Oncol Biol Phys 62 1283-90

Pignol J-P, Keller B, Rakovitch E, Sankreacha R, Easton H and Que W 2006 First report of a permanent breast $103 \mathrm{Pd}$ seed implant as adjuvant radiation treatment for early-stage breast cancer International journal of radiation oncology, biology, physics 64 176-81

Poon E, Williamson J F, Vuong T and Verhaegen F 2008 Patient-specific Monte Carlo dose calculations for high-dose-rate endorectal brachytherapy with shielded intracavitary applicator Int J Radiat Oncol Biol Phys 72 1259-66

Raina S, Avadhani J S, Oh M, Malhotra H K, Jaggernauth W, Kuettel M R and Podgorsak M B 2005 Quantifying IOHDR brachytherapy underdosage resulting from an incomplete scatter environment Int J Radiat Oncol Biol Phys 61 1582-6

Richardson S, Garcia-Ramirez J, Lu W, Myerson R J and Parikh P 2012 Design and dosimetric characteristics of a new endocavitary contact radiotherapy system using an electronic brachytherapy source Med Phys $396838-46$ 
Rivard M J, Coursey B M, DeWerd L A, Hanson W F, Huq M S, Ibbott G S, Mitch M G, Nath R and Williamson J F 2004 Update of AAPM Task Group No. 43 Report: A revised AAPM protocol for brachytherapy dose calculations Medical Physics 31 633-74

Rivard M J, Davis S D, DeWerd L A, Rusch T W and Axelrod S 2006 Calculated and measured brachytherapy dosimetry parameters in water for the Xoft Axxent X-Ray Source: an electronic brachytherapy source Med Phys 33 4020-32

Rong Y and Welsh J S 2010 Surface applicator calibration and commissioning of an electronic brachytherapy system for nonmelanoma skin cancer treatment Med Phys 37 5509-17

Sarrut D and Guigues L 2008 Region-oriented CT image representation for reducing computing time of Monte Carlo simulations Med Phys 35 1452-63

Schellhammer P F, El-Mahdi A M and Wakley J 1977 I-125 interstitial implantation of the prostate Va Med 104 $382-5$

Segala J J, Cardarelli G A, Hiatt J R, Curran B H and Sternick E S 2011 Interface dosimetry for electronic brachytherapy intracavitary breast balloon applicators J Appl Clin Med Phys 123221

Sharma R and Jursinic P A 2013 In vivo measurements for high dose rate brachytherapy with optically stimulated luminescent dosimeters Med Phys $\mathbf{4 0} 071730$

Tanderup K, Beddar S, Andersen C E, Kertzscher G and Cygler J E 2013 In vivo dosimetry in brachytherapy Med Phys $\mathbf{4 0} 070902$

Tedgren A C and Carlsson G A 2013 Specification of absorbed dose to water using model-based dose calculation algorithms for treatment planning in brachytherapy Phys Med Biol 58 2561-79

Thomadsen B R, Rivard M J and Butler W M 2005 Brachytherapy Physics, Second Edition. vol AAPM Medical Physics Monograph \#31: Medical Physics Publishing)

Uniyal S C, Sharma S D and Naithani U C 2012 A dosimetry method in the transverse plane of HDR Ir-192 brachytherapy source using gafchromic EBT2 film Phys Med 28 129-33

Vaidya J S, et al. 2001 Targeted intra-operative radiotherapy (Targit): an innovative method of treatment for early breast cancer Ann Oncol 12 1075-80

Walters B R, Kramer R and Kawrakow I 2010 Dose to medium versus dose to water as an estimator of dose to sensitive skeletal tissue Phys Med Biol 55 4535-46

White H W a D 1986 The composition of body tissues Br. J. Radiol. 591209

Ye S J, Brezovich I A, Shen S, Duan J, Popple R A and Pareek P N 2004 Attenuation of intracavitary applicators in 192Ir-HDR brachytherapy Med Phys 31 2097-106 



\section{Chapter 2: Influence of trace elements in human tissue in low energy photon brachytherapy dosimetry}

This work was part of an invited presentation at the "International Workshop on Recent Advances in Monte Carlo Techniques for Radiation Therapy", held in Montreal, June 8-10, 2011 


\begin{abstract}
Purpose

To determine the dosimetric impact of trace elements in human tissues for low energy photon sources used in brachytherapy.

\section{Methods and materials}

Monte Carlo dose calculations were used to investigate the dosimetric effect of trace elements present in normal or cancerous human tissues. The effect of individual traces (atomic number $\mathrm{Z}=11$ to 30 ) was studied in soft tissue irradiated by low energy brachytherapy sources. Three other tissue types (prostate, adipose and mammary gland) were also simulated with varying trace concentrations to quantify the contribution of each trace to the dose distribution. The dose differences between cancerous and healthy prostate tissues were calculated in single and multi-source geometries.
\end{abstract}

\title{
Results
}

The presence of traces in a tissue produces a difference in the dose distribution that is dependent on $\mathrm{Z}$ and the concentration of the trace. Low- $\mathrm{Z}$ traces $(\mathrm{Na})$ have a negligible effect $(<0.3 \%)$ in all tissues while higher $\mathrm{Z}(\mathrm{K})$ had a larger effect $(>3 \%)$. There is a considerable difference in the dose distribution between cancerous and healthy prostate tissues (4\%) and even larger if compared to the trace-free composition (15\%) in both single and multi-sourced geometries.

\section{Conclusion}

Trace elements have a non-negligible effect on the dose in tissues irradiated with low energy photon sources. This study underlines the need for further investigation into accurate determination of the trace composition of tissues associated with low energy brachytherapy. Alternatively, trace elements could be incorporated as a source of uncertainty in dose calculations. 


\section{Introduction}

Low energy photon brachytherapy is an increasingly popular form of radiotherapy in prostate cancer as low dose rate transperineal permanent seed implantation (Nath, 1995) and as a form of radiation boost in breast cancer patients (Pignol et al., 2006b). Low energy brachytherapy currently relies on the protocol of the AAPM Radiation Therapy Committee Task Group 43 (TG-43) (Nath, 1995; Rivard et al., 2004a) to perform dosimetric evaluations of brachytherapy implants and other procedures. The TG-43 protocol considers a uniform water geometry $30 \mathrm{~cm}$ in diameter with a source positioned at the center and uses dose kernels based on this geometry to calculate the dose in clinical scenarios. This calculation method works well for high energy sources such as Ir-192. However dose calculations for low energy sources are sensitive to a number of factors such as tissue heterogeneities, rendering this form of dose calculation inaccurate. Low energy photons $(<50 \mathrm{keV})$ interact primarily through the photoelectric effect, a process which is highly dependent on the composition of the medium and the energy of the incident photon.

The AAPM Radiation Therapy Committee Task Group 186 (TG-186) is investigating model based dose calculation algorithms as an alternative to the current TG-43 protocol. Monte Carlo (MC) calculations are a potential alternative due to their ability to accurately simulate low-energy photon interactions in realistic geometries. $\mathrm{MC}$ simulation methods require voxel-by-voxel knowledge of tissue density and composition. The accurate determination of composition data has been shown to be challenging due to the significant degree of overlap of Hounsfield Units between different media in modern CT scanners (Schneider et al., 2000). The elements found in tissues can be separated in two categories. Hydrogen, carbon, nitrogen and oxygen (HCNO) form the basis of soft tissues, each with concentrations in fractional weight greater than $1 \%$, accounting for over $99 \%$ of the total weight, while other higher atomic number $(\mathrm{Z})$ elements such as $\mathrm{Na}, \mathrm{S}, \mathrm{P}, \mathrm{Cl}$ and $\mathrm{K}$ are present at a trace level $(<1 \%)$. A recent study (Landry et al., 2010a) has demonstrated that uncertainties and variations in the HCNO human tissue composition could have a significant effect on MC dose distributions. In that work, compositions were varied according to population distributions reported by Woodard and White (Woodard and White, 1986), with no special attention to the significance of trace elements. No study has been found that assesses the specific contribution of traces to the MC dose calculation, which potentially could lead to uncertainty in the dose. As low energy photons such as those found in permanent implant brachytherapy interact mainly through the photoelectric effect, we hypothesize that dose distributions could be sensitive to changes in concentration of higher-Z $(>8)$ elements. Small inter-patient variation of trace element levels could thus contribute disproportionably to dosimetric uncertainty.

The trace composition of human tissues has been shown to vary considerably depending on the clinical state of the tissue such as the presence and stage of cancer (Carvalho et al., 2007a). If trace elements have an impact on the dose distribution, individual variations in the concentration could produce uncertainties in the dosimetry. Megavoltage photons interact primarily through Compton scattering which is Z-independent and hence the trace composition can be largely ignored for high energy radiotherapy.

A study was performed to investigate the importance of trace elements in low energy photon brachytherapy in healthy and cancerous human tissues using MC simulation techniques. 


\section{Methods and materials}

The software package used for the simulations in this study was Geant4 v9.3. Geant4 is an all purpose MC simulation toolkit that provides a diverse set of software components that can be employed in a variety of settings, including the simulation of photon transport from low energy sources (Agostinelli et al., 2003a). This paper uses the Lawrence Livermore National Laboratory low energy electromagnetic model which simulates the low energy photon interactions of the Photoelectric effect, Compton scattering and Rayleigh scattering. The model uses the EPDL (Cullen et al., 1997), and EADL (Perkins et al., 1997) evaluated libraries. All secondary electrons are made to deposit their energy locally due their short range compared to typical voxel sizes; hence dose is approximated by collision kerma. In view of the current debate over dose reporting (Ma and $\mathrm{Li}, 2011 \mathrm{a}$ ), both dose to medium $\left(D_{\mathrm{m}, \mathrm{m}}\right)$ and dose to water $\left(D_{\mathrm{w}, \mathrm{m}}\right)$ should be reported (both methods transport photons in medium (Landry et al., 2011)), but as this study is a demonstration, the majority of the results are reported in $D_{\mathrm{m}, \mathrm{m}}$.

\section{Composition of tissues}

The elemental constitution of the human tissues used in the first part of the study were taken from a commonly cited review paper on compositions (Woodard and White, 1986)unless otherwise stated. Four tissue types were used in this study (Table 1). These were selected due to their association with low energy brachytherapy. All compositions in table 1 were taken from healthy adult tissues. While it has been shown that uncertainties in the composition of HCNO can affect low energy brachytherapy (Landry et al., 2010a), the objective of this study is the investigation of the effects of trace element variations, and hence throughout the study the proportionality between $\mathrm{H}, \mathrm{C}, \mathrm{N}$, and $\mathrm{O}$ in each tissue remained fixed however the trace component varied. In addition to this, the density of each tissue was also kept constant.

In the later part of the study, a simulation was performed using a trace composition derived from a study which describes a series of trace compositions measured to correlate their concentration to tumor stage (Kwiatek et al., 2005). Among the tissues sampled was the prostate. The data provided describes the concentrations of trace elements ranging from $\mathrm{Cl}$ $(Z=17)$ to $\mathrm{Zn}(\mathrm{Z}=30)$. The effective atomic number $Z_{\text {eff }}$ for each of the compositions was also calculated by:

$$
Z_{\text {eff }}=\left(\sum_{i} w_{i} Z_{i}^{3.5}\right)^{1 / 3.5}
$$

Where $w_{i}$ is the mass fraction of the element $i$ with atomic number $Z_{i}$.

In the final part of the study the composition from Kwiatek et al was applied to the CT geometry of a CT scan of a patient who underwent brachytherapy seed implantation. 
Table 1: Composition of human body tissues relevant to low energy photon brachytherapy (derived from Woodard and White unless stated otherwise).

Elemental composition (by $\%$ weight)

\begin{tabular}{|c|c|c|c|c|c|c|c|c|c|c|}
\hline \multirow[b]{2}{*}{ Body tissue } & \multicolumn{4}{|c|}{ Element with $\mathrm{Z} \leq 8$} & \multicolumn{5}{|c|}{ Trace elements with $Z>8$} & \multirow[b]{2}{*}{ Density $/ \mathrm{kg} \mathrm{m}^{-3}$} \\
\hline & $\mathrm{H}$ & $\mathrm{C}$ & $\mathrm{N}$ & $\mathrm{O}$ & $\mathrm{Na}$ & $\mathrm{P}$ & $\mathrm{S}$ & $\mathrm{Cl}$ & $\mathrm{K}$ & \\
\hline soft tissue* & 10.1 & 11.1 & 2.6 & 76.2 & - & - & - & - & - & 1000 \\
\hline prostate & 10.5 & 8.9 & 2.5 & 77.4 & 0.2 & 0.1 & 0.2 & - & 0.2 & 1040 \\
\hline $\begin{array}{l}\text { mammary } \\
\text { gland }\end{array}$ & 10.6 & 33.2 & 3.0 & 52.7 & 0.1 & 0.1 & 0.2 & 0.1 & - & 1020 \\
\hline adipose & 11.4 & 59.8 & 0.7 & 27.8 & 0.1 & - & 0.1 & 0.1 & - & 950 \\
\hline
\end{tabular}

*(ICRU Report 46, 1992)

\section{Simulations}

Using Geant4 v9.3, a spherical phantom $20 \mathrm{~cm}$ in radius was modeled. The phantom contained a selected uniform composition and had a low energy isotropic point source placed at the center. The source had the photon spectral characteristics of one of three clinical sources, the 2301 I-125 source, the 2335 Pd-103 source (Best Medical, Springfield VA) and the Xoft Axxent electronic brachytherapy source (Xoft, Sunnyvale, CA) operated at $50 \mathrm{kV}$. The spectral characteristics of the Best seeds and Axxent source were derived using MC techniques (Landry et al., 2010a; Liu et al., 2008)

The source chosen depended on the clinical relevance it had to the tissue type, with 2301 (I$125)$ and 2335 (Pd-103) seed models being used in permanent seed prostate brachytherapy (Machtens et al., 2006) and the Axxent source and Pd-103 seed used in breast brachytherapy (Hepel et al., 2010b; Pignol et al., 2006b). All MC simulations calculated the dose per particle in $1 \mathrm{~mm}$ thick concentric spherical shells with the mid-radius of the first shell at 0.1 $\mathrm{cm}$ and last shell at $10 \mathrm{~cm}$ with the inverse square law dependence factored out (Landry et al, 2010). Simulating $10^{6}$ photons reduced the relative statistical uncertainty to less than $1 \%$ within a distance of $10 \mathrm{~cm}$ of the source (Landry et al., 2010a).

\section{$0.2 \%$ high-Z composition}

Using the soft tissue composition from table 1, the MC phantom was filled with a fictitious medium where $0.2 \%$ of the composition was re-allocated to a single trace element with the soft tissue's HCNO ratio remaining fixed. A total of 8 high-Z constituents were used (Table 2). The traces were chosen based on two reviews (Carvalho et al., 2007a; Woodard and White, 1986), where these elements were the most abundant traces in tissues. Two special cases were also simulated; one excluding all traces $(0 \%)$ and another including all the traces given an equal percentage to a total of $0.2 \%$. $0.2 \%$ was chosen as it approximately corresponds to the weight fraction of the traces in several human tissues of several of the traces (Woodard and White, 1986). However it is likely an overestimation of the 
concentration of other less abundant high- $\mathrm{Z}$ traces like $\mathrm{Zn}$ and $\mathrm{Fe}$ as well as an underestimation of the concentration of more abundant traces like $\mathrm{Na}, \mathrm{P}$ and $\mathrm{K}$.

Table 2: Soft tissue with a $0.2 \%$ trace component.

\begin{tabular}{|l|l|l|l|l|l|}
\hline Scenario & \multirow{2}{*}{$\begin{array}{l}\text { Fraction by } \\
\text { weight of } \\
\text { trace (\%) }\end{array}$} & \multicolumn{2}{l|}{ HNCO (\%) } \\
\cline { 5 - 7 } & H & C & N & O \\
\hline $\begin{array}{l}\text { Single trace element: Na , P, S, Cl, K, Ca, Fe } \\
\text { Zn }\end{array}$ & 0.2 & 10.0798 & 11.0778 & 2.5948 & 76.0476 \\
\hline All traces present (Na-Zn) & $0.2(0.025 \times 8)$ & 10.0798 & 11.0778 & 2.5948 & 76.0476 \\
\hline HCNO only & - & 10.1 & 11.1 & 2.6 & 76.2 \\
\hline
\end{tabular}

\section{Prostate, adipose and mammary gland}

For simulating prostate, adipose and mammary gland, published tissue compositions listed in table 1 were used. The trace elements component for each tissue was modified by excluding one of the traces at a time and redistributing the percentage to HCNO accordingly. The aim of the exclusion was to determine the individual contribution of each trace to the dose distribution. The calculation was also performed for the exclusion of all traces. Differences in dose per particle for trace variations were assessed by first comparing inverse square law corrected radial dose profiles.

The differences were also quantified by plotting the mean dose deposited in a spherical volume against incremental increase to the radius (R) of the sphere, from a sphere of $\mathrm{R}=$ $0.1 \mathrm{~cm}$ to a sphere with a radius of $10 \mathrm{~cm}$. Each mean dose value for successive radii was compared for all compositions and the percentage differences between them and the reference composition analyzed. This method allows for a more realistic representation of the dosimetric impact of trace compositions in a 3-D volume, rather than using a simple 1-D radial dose function profile which does not take into account the geometric effects on dose distributions.

\section{Healthy versus cancerous prostate tissue}

No recent comprehensive study into tissue composition with a full spectrum of elements could be found. However many studies into the concentration of trace elements present in human tissue have been done (Carvalho et al., 2007a; Kwiatek et al., 2005; Sarafanov et al., 2008; Zaichick and Zaichick, 2012). These studies investigate variations in concentrations of traces for the purpose of diagnosing the presence and stage of cancer. Of particular interest is the variation in the concentration of traces between healthy and cancerous tissue in the prostate (Kwiatek et al., 2005). The majority of the elemental composition, the HCNO component, was not reported. Kwiatek et al. reported the average trace concentrations of each element in healthy prostate tissue, prostate tissue classified as adenocarcinoma with Gleason score 5 and one with Gleason score 3 All samples were taken from healthy and cancerous segments of the prostate of six cancer patients.. Only the Gleason score 5 (CA-5) and healthy prostate tissue trace concentrations were used in our study. The Woodard and White prostate ( $W \& W$ prostate) was updated to include the new trace data (Table 3 ). The trace composition was added at the equal expense of the HCNO components with the ratio between $\mathrm{H}, \mathrm{C}, \mathrm{N}$, and $\mathrm{O}$ maintained in order to ensure a fair comparison to other simulations. Kwiatek et al. did 
not include some trace elements that were present in the $W \& W$ prostate and so these elements were not adjusted or removed. Of all the traces measured in both studies, there was only one overlap, potassium $(\mathrm{K})$ where both papers reported different percentages. In such cases of conflicting data, Kwiatek et al. values were used. Each composition from table 3 was simulated. The method used to determine the trace concentration in Kwiatek et al, synchrotron radiation induced X-ray emission (SRIXE), is described as having a statistical uncertainty of $15-20 \%$ in a related paper (Kwiatek et al., 2001). Kwiatek et al's (2005) reports large standard deviations in the concentrations recorded in the average of all the patients due to the variations between patients and though the standard deviations among some of the concentrations can be as large as $20 \%$ for some of more abundant traces, we feel that given the exploratory nature of this study, our chosen concentrations are acceptable. As with the previous section, the dose per particle profiles were integrated over a spherical volume and the percentage difference, relative to the $W \& W$ prostate, was plotted against the radii of the integrating spherical volume.

Table 3: Composition and effective atomic number (zeff) of four healthy and cancerous prostate tissues with varying trace concentrations.

\begin{tabular}{|l|l|l|l|l|}
\cline { 2 - 5 } \multicolumn{1}{c|}{} & $\begin{array}{l}\text { W\&W prostate } \\
\text { (Woodard and White, } \\
1986)\end{array}$ & $\begin{array}{l}\text { No traces } \\
\text { (HCNO only) }\end{array}$ & $\begin{array}{l}\text { Healthy prostate } \\
\text { (Kwiatek et al., 2005) }\end{array}$ & $\begin{array}{l}\text { Cancerous prostate } \\
\text { (Prostate CA-5) } \\
\text { (Kwiatek et al., } 2005)\end{array}$ \\
\hline Element & $\%$ & $\%$ & $\%$ & $\%$ \\
\hline $\mathrm{H}$ & 10.500 & 10.574 & 10.482 & 10.4558 \\
$\mathrm{C}$ & 8.900 & 8.962 & 8.885 & 8.8625 \\
$\mathrm{~N}$ & 2.500 & 2.517 & 2.495 & 2.4894 \\
$\mathrm{O}$ & 77.400 & 77.945 & 77.272 & 77.0742 \\
& & & & \\
$\mathrm{Na}$ & 0.2000 & - & 0.2000 & 0.2000 \\
$\mathrm{P}$ & 0.1000 & - & 0.1000 & 0.1000 \\
$\mathrm{~S}$ & 0.2000 & - & 0.2000 & 0.2000 \\
$\mathrm{~K}$ & 0.2000 & - & 0.1475 & 0.3620 \\
$\mathrm{Cl}$ & - & - & 0.18000 & 0.18300 \\
$\mathrm{Ca}$ & - & - & 0.01200 & 0.04100 \\
$\mathrm{Zn}$ & - & - & 0.01580 & 0.01760 \\
$\mathrm{Fe}$ & - & - & 0.00520 & 0.01145 \\
$\mathrm{Cu}$ & - & - & 0.00127 & 0.00121 \\
$\mathrm{Co}$ & - & - & 0.00038 & 0.00054 \\
$\mathrm{Ni}$ & - & - & 0.00045 & 0.00051 \\
$\mathrm{Mn}$ & - & - & 0.00018 & 0.000345 \\
$\mathrm{~V}$ & - & - & 0.000041 & 0.000202 \\
$\mathrm{Cr}$ & - & 7.34 & 7.53 & 0.000087 \\
\hline $\mathrm{Z}$ Effective & 7.50 & & 7.63 \\
\hline
\end{tabular}

\section{Effect of trace composition in a multi-source patient geometry}

Of clinical interest is the dosimetric impact that trace concentration variation could have on a realistic patient geometry with multiple sources. A previous study (Landry et al., 2010a) had described the effect of HCNO variation in a multi-source geometry. In Landry et al, 4 postimplant CTs of prostate brachytherapy seed patients were converted into a voxelized 
geometry using an in-house CT image conversion tool, BrachyGUI (Poon et al., 2008a). The CT images gave a complete visualization of the prostates, which were contoured by a clinician. During the voxelization each voxel was assigned adjustable elemental composition and the corresponding density. As the tumour volume could not be delineated on the CT images the entire prostate was assigned a single composition. The number of seeds and their position were measured using Seedfinder (VariSeed,Varian, Palo Alto, CA ). In the case of this study the patients had between 51 and 99 I-125 2301 seeds implanted.

In our study, each composition from table 3 was simulated as the prostate composition for each patient. In all cases the remaining voxels outside of the prostate were assigned as water. A separate simulation using a prostate with a water composition was also simulated to enable a comparison to TG-43 parameters. In all cases the inter-seed attenuation was ignored by using a phase space file as the particle source. The minimal dose to $90 \%$ of the prostate volume $\left(\mathrm{D}_{90}\right)$ in each simulation was calculated post-simulation using BrachyGUI.

\section{Results and discussion}

For all of the MC simulations, the results were plotted with $D_{\mathrm{m}, \mathrm{m}}$ per photon against radial distance from the source. The dose was multiplied by the square of the radial distance to factor out the inverse square law to clarify the effect of the traces.

\section{Effect of trace elements in soft tissue}

\section{soft tissue with $0.2 \%$ trace}
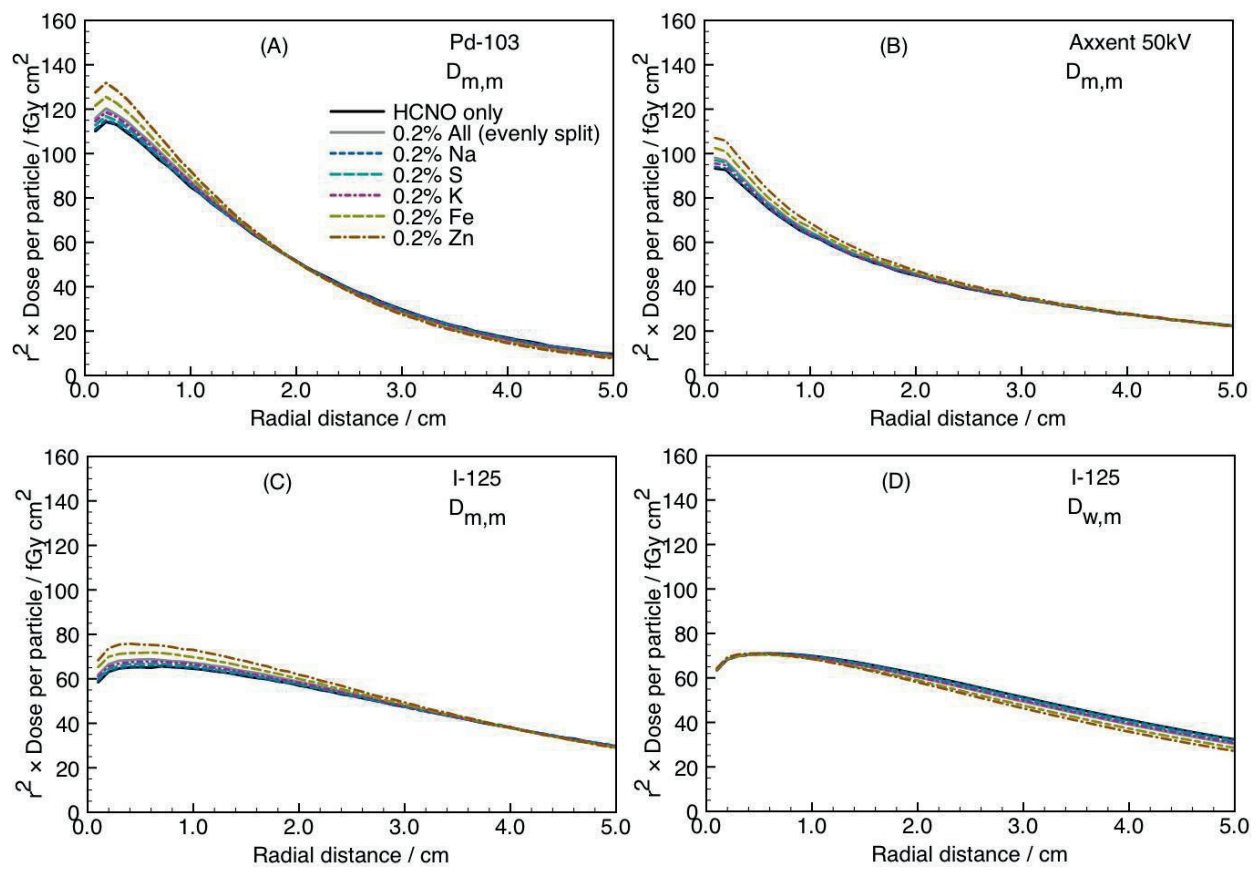

Figure 1: The dose profile for $D_{\mathrm{m}, \mathrm{m}}$ for Pd-103 (A), Axxent (B) and I-125 (C) sources in soft tissue using a varying $\mathrm{Z}$ trace. Different dose reporting methods are shown for an I-125 source as $D_{\mathrm{m}, \mathrm{m}}(\mathrm{C})$ and $D_{\mathrm{w}, \mathrm{m}}(\mathrm{D})$. 
As can be seen in Figures 1 A-C for the three brachytherapy sources the lower Z trace elements such as $\mathrm{Na}$ and $\mathrm{S}$ have a smaller effect on the dose profile than higher $\mathrm{Z}$ elements like $\mathrm{Zn}$ and $\mathrm{Fe}$ for a $0.2 \%$ weight concentration, due to their lower photoelectric effect cross section. The $D_{\mathrm{m}, \mathrm{m}}$ from the I-125 and Pd-103 sources show a Z-dependent maximum within a few millimeters from the source. In figure $1 \mathrm{~A}$, the Pd-103 source exhibits a sharp peak and steep falloff. The I-125 source exhibits a flatter Z-dependent dose falloff due to the slightly higher photon energy of this isotope compared to Pd-103. The Axxent source has its maximum in the first shell $(0-1 \mathrm{~mm})$. Both brachytherapy radioisotopes have a discrete spectrum while the Axxent source has a continuous spectrum. As can be seen in the figure from A-C the differences in the appearance of all graphs demonstrate the strong spectral dependence on the dose profile, and the varying magnitude that different traces has on them. In general, and as expected, the addition of higher $\mathrm{Z}$ elements causes $D_{\mathrm{m}, \mathrm{m}}$ to increase near the source. Due to the increased attenuation, the dose gradient also becomes steeper with increasing $\mathrm{Z}$ leading all the profiles in each figure to cross-over at a certain distance from the source

Figures 1CD show the difference between $D_{\mathrm{w}, \mathrm{m}}$ and $D_{\mathrm{m}, \mathrm{m}}$ for an I-125 source for different traces. The dose pattern is different when scoring $D_{\mathrm{w}, \mathrm{m}}$ with lower $\mathrm{Z}$ compositions. The slopes are similar between $D_{\mathrm{w}, \mathrm{m}}$ and $D_{\mathrm{m}, \mathrm{m}}$ but the dose in the first shell differs by the ratio of mass energy absorption coefficients. The difference occurs because the photons are transported and attenuated in the medium but $D_{\mathrm{w}, \mathrm{m}}$ is scored in water. For all cases the photon fluence close to the source can be assumed to be the same, when scoring $D_{\mathrm{w}, \mathrm{m}}$ the mass energy absorption coefficients of water are used, resulting in almost identical doses close to the source (Fig 1D). For scoring $D_{\mathrm{m}, \mathrm{m}}$ the photon fluence close to the source is effectively multiplied by different mass energy absorption coefficients for different media, resulting in different doses (Fig 1A-C). This section shows that dose differences caused by traces can be quite different close to the source for $D_{\mathrm{m}, \mathrm{m}}$ or at larger distances if $D_{\mathrm{w}, \mathrm{m}}$ is considered. It is therefore important to report the dose scoring scheme used.

\section{Effect of trace elements in prostate, adipose and mammary gland}

\section{Prostate}

The results for the MC simulation in prostate are found in figure 2. The removal of the lower $\mathrm{Z}$ component, $\mathrm{Na}$, from the prostate composition has a relatively small effect $(<0.5 \%)$ on the dose profile while the removal of higher $Z$ ( $S$ and $K$ ) reduce the peak dose per particle by $4 \%$. If all traces are removed this difference increases to $7 \%$ (Fig 2A). The difference between the curves decreases with increasing distance to a crossover point before they increase again in the opposing direction.

Figure 2B shows the mean dose $D_{\mathrm{m}, \mathrm{m}}$ in spheres of increasing radius centered on the source . It can be seen that the relative difference between compositions is greatest close to the source. Also it can be seen that low $\mathrm{Z}$ elements have an almost negligible effect on the dose per particle for all distances as opposed to higher $\mathrm{Z}$ materials. The absence of all traces has the largest impact on the average dose per particle in a volume, as more of the photons pass through the medium unattenuated. This shows that the presence of the high $\mathrm{Z}$ elements induces a greater energy deposition as a function of distance. It should be noted that the 
difference in integrated energy in all cases approaches zero at a large enough distance due the conservation of energy (i.e. the total energy deposited will eventually be equal).
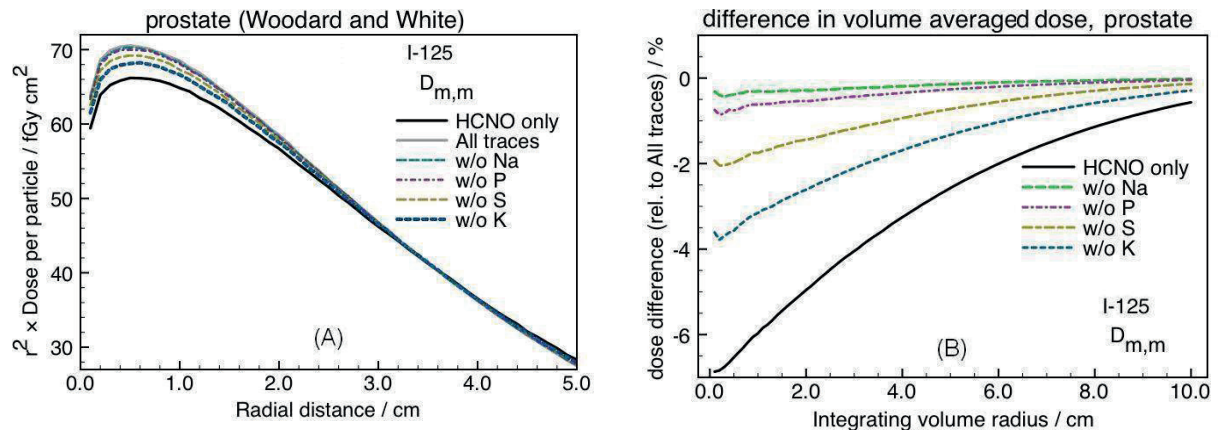

Figure 2: (A) The $D_{\mathrm{m}, \mathrm{m}}$ profiles of the I-125 source in prostate tissue with and without trace elements. (B) The dose difference between each variation, relative to all traces, integrated over a spherical volume.
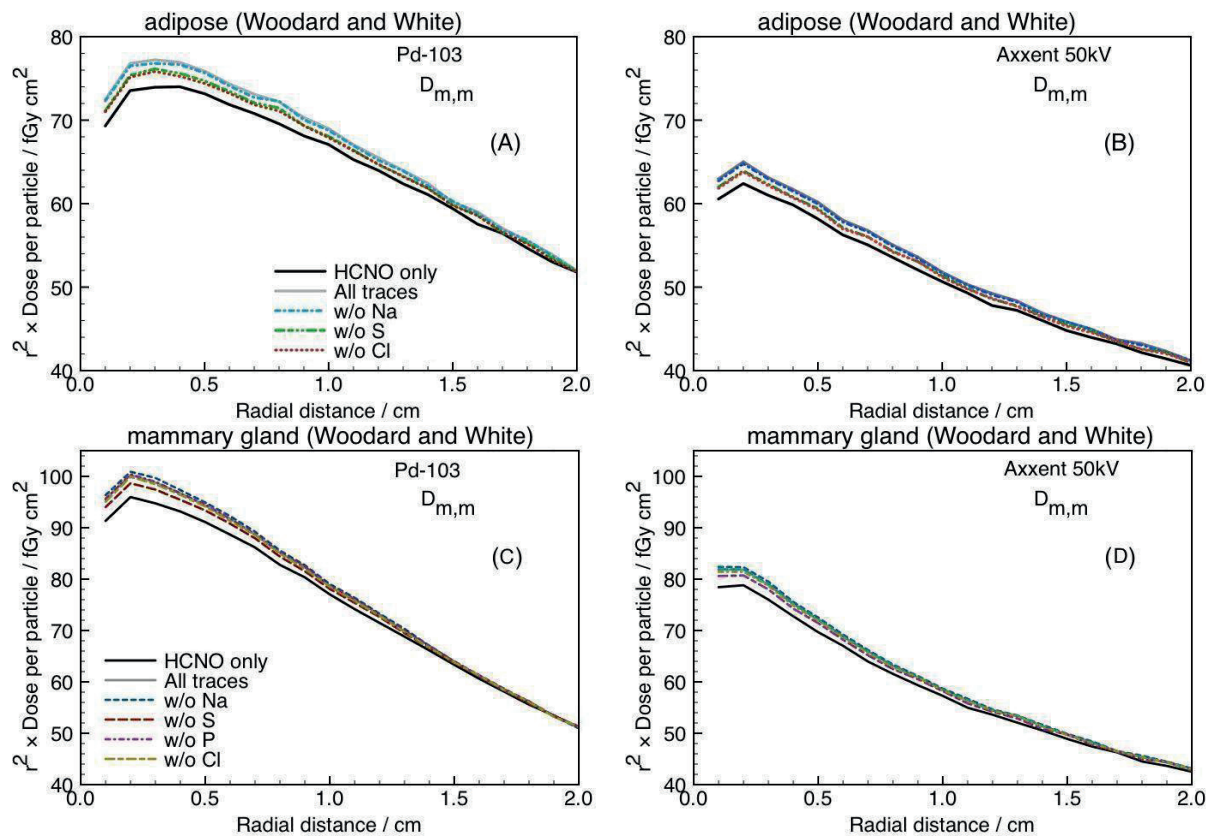

Figure 3: $D_{\mathrm{m}, \mathrm{m}}$ profiles in adipose for (A) Pd-103 and (B) Axxent sources and in mammary gland (C) Pd103 and (D) Axxent with and without trace elements

Figure $3 \mathrm{AB}$ shows the dose profile for two sources in adipose for varying trace elements. The Pd-103 seed and the Axxent source demonstrate similar profiles in both figures, with a similar response to the removal of trace elements. The maximum percentage difference between All traces and HCNO only in figure $3 \mathrm{AB}$ is similar for both sources (4\% for Pd-103 and $4 \%$ for the Axxent). 
Figure 3CD for mammary gland possesses the same characteristics of the two other tissues prostate and adipose with a gradual decrease in the maximum of the dose profiles as higher $\mathrm{Z}$ traces are removed. The maximum difference between the HCNO only and all traces is 5\% and 4\% for Pd-103 and the Axxent respectively. Adipose has a lower trace content than mammary gland but has a similar maximum percentage difference when the traces are removed due to adipose's lower average $\mathrm{Z}$ compared to mammary gland. This gives adipose a greater sensitivity to changes in the trace composition. It has also been shown that the composition of adipose tissue can vary significantly between samples and so the effect that each trace has on the dose can vary (Landry et al., 2010a). It is worth noting that as real breast tissue is a complex mixture of adipose and mammary tissue (Nelson et al., 2008) and not homogenous as in this case, the results from these tissues are not based on a realistic case. A more detailed study should use a composition based on a segmented breast geometry such as those described in Afsharpour et al and Nelson et al (Afsharpour et al., 2011b). However our study demonstrates clearly that traces have a non-negligible effect on dose distributions in addition to the uncertainties related to tissue segmentation

\section{Healthy versus cancerous prostate tissue}

Figure $4 \mathrm{AB}$ shows the different dose profiles of four prostate compositions and the mean dose differences, relative to $W \& W$ prostate, when they are integrated over a spherical volume. It is worth noting, in figure $4 \mathrm{~B}$, the difference between prostate $C A-5$ and $H C N O$ only of $15 \%$ at $0.5 \mathrm{~cm}$. Another notable difference is the variation between Kwiatek et al.'s healthy prostate and cancerous prostate $C A-5$ dose profiles which has a maximum difference of $4 \%$ at $0.5 \mathrm{~cm}$. This shows that the trace concentration difference between healthy and cancerous tissue may be large enough to produce a considerable dose difference. It should be mentioned that Kwiatek et al. measured only certain trace elements (missing most notably $\mathrm{Na}, \mathrm{P}$ and $\mathrm{S}$ ) and therefore, their data may be incomplete. This may cause an unquantifiable systematic error and as such the results from this section should not be seen to quantify the exact differences in dose profiles between healthy and cancerous tissue, but it should demonstrate that potential changes in trace concentration may have a non-negligible effect on dose distributions. A cancerous prostate is also not composed entirely of cancerous tissue and is actually a mixture of both healthy and cancerous tissue. This constitutes another source of uncertainty as a CT image cannot make any distinction between cancerous and healthy tissue. However advances in dual energy CT (DECT) may allow for better delineation of prostate tissue or perhaps even a elemental breakdown of the tissue itself (Liu et al., 2009). Kwiatek et al also possesses large standard deviations in its measured trace compositions due to inter and intra patient composition variations as well as uncertainties with the measurement modality, SRIXE. However this study is merely a demonstration and the uncertainties and variations emphasize the necessity for further study. 

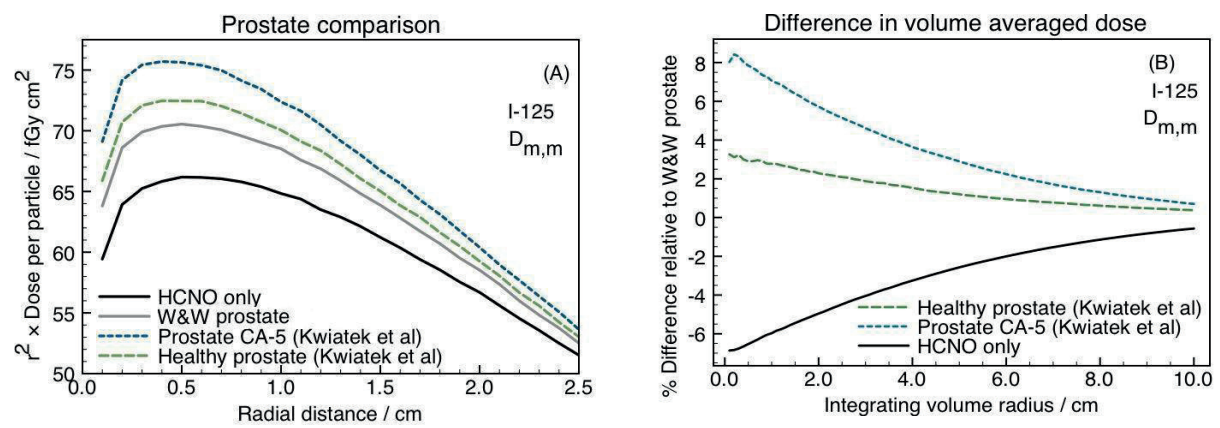

Figure 4: (A) $D_{\mathrm{m}, \mathrm{m}}$ profiles in four prostate tissues using different trace compositions derived from Woodard and White (W\&W) and Kwiatek et al. (B) The difference in mean dose between the different tissues, relative to $W \& W$ prostate, over a spherical volume.

\section{Effect of trace composition in a multi-source patient geometry}

Table 4 shows the $\mathrm{D}_{90}$ for various compositions, in absolute and relative to a water composition. The $\mathrm{D}_{90}$ for all 4 prostate compositions from table 3 increase with increasing trace composition with a maximum difference of $9-12 \%$ (14-16Gy) between a zero-trace composition (No traces) and cancerous prostate tissue (Prostate CA-5) for all patients.

It can be seen here that trace elements can cause a notable dose variation, not only in single source geometries but also in a multi-source clinical scenario. However there are some significant sources of uncertainty in this study. Again these simulations assume a prostate of uniform composition of either healthy or cancerous tissue, which is unrealistic, but is a necessary assumption if only CT images are available. Among the other heterogeneities that are present in the prostate are calcifications, small regions with a high concentration of calcium, observable on CT and ultrasound. Calcifications are not present in all prostates and can be confused for seeds and so constitute a source of uncertainty. It is unknown how calcification affects the concentration of calcium in the whole prostate but the effects of calcifications on the dose distribution have been explored (Chibani and Williamson, 2005) .

Table 4: The $\mathrm{D}_{90}$ for the 4 prostate compositions, in absolute and relative to a water-based composition

\begin{tabular}{|c|c|c|c|c|c|c|}
\hline $\begin{array}{l}\text { Patient/ } \\
\text { No. of seeds }\end{array}$ & Composition & Water & $\begin{array}{l}\text { W\&W } \\
\text { prostate }\end{array}$ & No traces & $\begin{array}{l}\text { Healthy prostate } \\
\text { (Kwiatek et al) }\end{array}$ & $\begin{array}{l}\text { Cancerous prostate } \\
\text { (Kwiatek et al) }\end{array}$ \\
\hline \multirow{2}{*}{$\begin{array}{l}\text { Patient } 1 \\
\mathrm{~N}=75\end{array}$} & $\mathrm{D}_{90}$ (Gy) & 141.6 & 140.1 & 133.2 & 142.5 & 146.4 \\
\hline & $\%$ of Water & 100 & 98.9 & 94 & 100.6 & 103.4 \\
\hline \multirow{2}{*}{$\begin{array}{l}\text { Patient } 2 \\
\mathrm{~N}=77\end{array}$} & $\overline{D_{90}}$ (Gy) & 154.3 & 152.6 & 144.6 & 155.9 & 160.4 \\
\hline & $\%$ of Water & 100 & 98.9 & 93.7 & 101 & 104.5 \\
\hline \multirow{2}{*}{$\begin{array}{l}\text { Patient } 3 \\
\mathrm{~N}=51\end{array}$} & $D_{90}(G y)$ & 145.3 & 143.9 & 136 & 146.2 & 151.2 \\
\hline & $\%$ of Water & 100 & 99 & 93.6 & 100.6 & 104 \\
\hline \multirow{2}{*}{$\begin{array}{l}\text { Patient } 4 \\
\mathrm{~N}=99\end{array}$} & $D_{90}(G y)$ & 146.1 & 144.2 & 137.4 & 146.4 & 150.1 \\
\hline & $\%$ of Water & 100 & 98.6 & 94 & 100.2 & 102.7 \\
\hline
\end{tabular}




\section{Conclusion}

Dose distributions were obtained from MC simulations for low energy brachytherapy sources in human tissues with varying trace element content. The simulations, scored with dose to medium $D_{\mathrm{m}, \mathrm{m}}$, show a strong dependence to the $\mathrm{Z}$ and the concentration of the trace present. The absence of low $Z$ traces like $\mathrm{Na}$ from a tissue has a negligible effect on the dose calculation when compared to higher $\mathrm{Z}$ traces like Fe and $\mathrm{Zn}$. The effect of a trace is also dependent on the mean energy and photon spectrum of the source being simulated, with lower mean energy sources exhibiting a greater sensitivity. Results from this study show that the trace component of a tissue is not negligible in low energy brachytherapy MC calculation and therefore should not be excluded from $\mathrm{MC}$ calculations. The variation in trace concentration between healthy and cancerous prostate tissue was shown to alter the dose distributions.

Further study of the trace elements in human healthy and cancerous tissues is needed, as well as studies into the potential variability between compositions in a population of patients. While it may be possible that the next generation CT scanners will be able to determine the tissue composition, it is unlikely that trace component will be measured to a high (or any) level of accuracy nor that patients will have individual biopsies to determine the elemental composition of their tissues. As such, the potential dose uncertainties caused by trace element composition uncertainties should be quantified.

\section{Acknowledgments:}

This work was supported by a Maastro-Atrium grant (2009-12) on Assessment of dose-effect relationship between radiation dose and complications in prostate brachytherapy and by Grants Nos. 017133 and 700810 of the Canadian Cancer Society.

GL is supported by a PGSD2 scholarship from the Natural Sciences and Engineering Research Council of Canada and by the O'Brien Foundation (New Brunswick, Canada). BR was supported by a Marie Curie Reintegration grant (no PIRG05-GA-2009-247878).

\section{References}

Afsharpour H, Landry G, Reniers B, Pignol J P, Beaulieu L and Verhaegen F 2011 Tissue modeling schemes in low energy breast brachytherapy Physics in Medicine and Biology 56 7045-45

Agostinelli S, et al. 2003 Geant4,a simulation toolkit Nuclear Instruments and Methods in Physics Research Section A: Accelerators, Spectrometers, Detectors and Associated Equipment 506 250-303

Carvalho M L, Magalhaes T, Becker M and von Bohlen A 2007 Trace elements in human cancerous and healthy tissues: A comparative study by EDXRF, TXRF, synchrotron radiation and PIXE Spectrochimica Acta Part B: Atomic Spectroscopy 62 1004-11

Chibani O and Williamson J F 2005 MCPI[sup (Copyright)]: A sub-minute Monte Carlo dose calculation engine for prostate implants Medical Physics 32 3688-98

Cullen D, Hubbell J H and Kissel L 1997 EPDL97: the Evaluated Photon Data Library, 97 version UCRL-50400 Vol. 6, Rev 5

Hepel J T, Hiatt J R, Cardarelli G A and Wazer D E 2010 Modeling study for optimization of skin dose for partial breast irradiation using Xoft Axxent electronic brachytherapy applicator Brachytherapy 9 81-85 
Kwiatek W M, Banas A, Gajda M, Galka M, Pawlicki B, Falkenberg G and Cichocki T 2005 Cancerous tissues analyzed by SRIXE Journal of Alloys and Compounds 401 173-77

Kwiatek W M, Kubica B, Paluszkiewicz C and Galka M 2001 Trace element analysis by means of synchrotron radiation, XRF, and PIXE: selection of sample preparation procedure Journal of Alloys and Compounds 328 283-88

Landry G, Reniers B, Murrer L, Lutgens L, Bloemen-Van Gurp E, Pignol J, Keller B, Beaulieu L and Verhaegen F 2010 Sensitivity of low energy brachytherapy Monte Carlo dose calculations to uncertainties in human tissue composition Med. Phys. 37 5188-98

Landry G, Reniers B, Pignol J P, Beaulieu L and Verhaegen F 2011 The difference of scoring dose to water or tissues in Monte Carlo dose calculations for low energy brachytherapy photon sources Med Phys $\mathbf{3 8}$ 1526-33

Liu D, Poon E, Bazalova M, Reniers B, Evans M, Rusch T and Verhaegen F 2008 Spectroscopic characterization of a novel electronic brachytherapy system Phys Med Biol $\mathbf{5 3}$ 61-75

Liu X, Yu L, Primak A N and McCollough C H 2009 Quantitative imaging of element composition and mass fraction using dual-energy CT: Three-material decomposition Medical Physics 36 1602-02

Ma C M and Li J 2011 Dose specification for radiation therapy: dose to water or dose to medium? Phys Med Biol 56 3073-89

Machtens S, Karstens J H, Baumann R and Jonas U 2006 Interstitial Brachytherapy (LDR-Brachytherapy) in the Treatment of Patients with Prostate Cancer European Urology Supplements 5 514-21

Nath R 1995 Dosimetry of interstitial brachytherapy sources: Recommendations of the AAPM Radiation Therapy Committee Task Group No. 43 Med. Phys. 22 209-34

Nelson T R, Cervino L I, Boone J M and Lindfors K K 2008 Classification of breast computed tomography data Med Phys 35 1078-86

Perkins S T, Cullen D E, Chen M H, Hubbell J H, Rathkopf J and Scofield J 1997 Table and graphs of atomic subshell and relaxation data derived from the LLNL Evaluated Atomic Data Library (EADL) UCRL50400 Vol. 30

Pignol J-P, Keller B, Rakovitch E, Sankreacha R, Easton H and Que W 2006 First report of a permanent breast $103 \mathrm{Pd}$ seed implant as adjuvant radiation treatment for early-stage breast cancer International Journal of Radiation Oncology*Biology*Physics 64 176-81

Poon E, Le Y, Williamson J F and Verhaegen F 2008 BrachyGUI: an adjunct to an accelerated Monte Carlo photon transport code for patient-specific brachytherapy dose calculations and analysis Journal of Physics: Conference Series 102012018

Rivard M J, Coursey B M, DeWerd L A, Hanson W F, Huq M S, Ibbott G S, Mitch M G, Nath R and Williamson J F 2004 Update of AAPM Task Group No. 43 Report: A revised AAPM protocol for brachytherapy dose calculations Med Phys 31 633-74

Sarafanov A G, Todorov T I, Kajdacsy-Balla A, Gray M A, Macias V and Centeno J A 2008 Analysis of iron, zinc, selenium and cadmium in paraffin-embedded prostate tissue specimens using inductively coupled plasma mass-spectrometry Journal of Trace Elements in Medicine and Biology 22 305-14

Schneider W, Bortfeld T and Schlegel W 2000 Correlation between CT numbers and tissue parameters needed for Monte Carlo simulations of clinical dose distributions Phys Med Biol 45 459-78

Woodard H Q and White D R 1986 The composition of body tissues Br J Radiol 59 1209-18

Zaichick S and Zaichick V 2012 Trace elements of normal, benign hypertrophic and cancerous tissues of the Human prostate gland investigated by neutron activation analysis Applied Radiation and Isotopes $\mathbf{7 0}$ $81-87$ 


\section{Chapter 3: \\ Comparison of TG-43 and TG-186 in breast irradiation using a low energy electronic brachytherapy source}

Shane A. White, Guillaume Landry, Gabriel Paiva Fonseca, Randy Holt, Thomas Rusch, Luc Beaulieu, Frank Verhaegen, Brigitte Reniers. 


\begin{abstract}
Purpose

The recently updated guidelines for dosimetry in brachytherapy in TG-186 have recommended the use of model-based dosimetry calculations as a replacement for TG-43. TG-186 highlights shortcomings in the water-based approach in TG-43, particularly for low energy brachytherapy sources. The Xoft Axxent is a low energy $(<50 \mathrm{kV})$ brachytherapy system used in accelerated partial breast irradiation (APBI). Breast tissue is a heterogeneous tissue in terms of density and composition. Dosimetric calculations of 7 APBI patients treated with Axxent were made using a model-based Monte Carlo platform for a number of tissue models and dose reporting methods and compared to TG-43 based plans.
\end{abstract}

\title{
Methods and materials
}

A model of the Axxent source, the S700, was created and validated against experimental data. CT scans of the patients were used to create realistic multi-tissue/heterogeneous models with breast tissue segmented using a published technique. Alternative water models were used to isolate the influence of tissue heterogeneity and backscatter on the dose distribution. Dose calculations were performed using Geant4 according to the original treatment parameters. The effect of the Axxent balloon applicator used in APBI which could not modeled in the CT-based model, was modeled using a novel techniques that utilize CADbased geometries. These techniques were validated experimentally. Results were calculated using two dose reporting methods, dose to water $\left(D_{w, m}\right)$ and dose to medium $\left(D_{m, m}\right)$, for the heterogeneous simulations. All results were compared against TG-43-based dose distributions and evaluated using dose ratio maps and DVH metrics. Changes in skin and PTV dose were highlighted

\section{Results}

All simulated heterogeneous models showed a reduced dose to the DVH metrics that is dependent on the method of dose reporting and patient geometry. Based on a prescription dose of $34 \mathrm{~Gy}$, the average $\mathrm{D}_{90}$ to PTV was reduced by between $\sim 4 \%$ and $\sim 40 \%$, depending on the scoring method, compared to the TG-43 result. Peak skin dose is also reduced by $10-$ $15 \%$ due to the absence of backscatter not accounted for in TG-43. The balloon applicator also contributed to the reduced dose. Other ROIs showed a difference depending on the method of dose reporting.

\section{Conclusions}

TG-186-based calculations produce results that are different from TG-43 for the Axxent source. The differences depend strongly on the method of dose reporting. This study highlights the importance of backscatter to peak skin dose. Tissue heterogeneities, applicator and patient geometries demonstrate the need for a more robust dose calculation method for low energy brachytherapy sources. 


\section{Introduction}

Low-energy photon brachytherapy is an established form of radiotherapy that is used in the treatment of prostate cancer(Nath et al., 1995) and with potential for extensive use in breast cancer(Pignol et al., 2006a). These forms of cancer treatment utilize millimeter size seeds that encapsulate a photon-emitting radioactive material adsorbed to the interior of the seed. Low energy HDR breast brachytherapy is nowadays possible with an electronic brachytherapy $(\mathrm{eBx})$ source(Dickler, 2009) which delivers radiation using a miniaturized xray source.

The recently published AAPM Radiation Therapy Committee Task Group 186 (TG186)(Beaulieu et al., 2012) report has provided new guidelines for dose calculation and reporting in brachytherapy to address the shortcomings of the current TG-43U1(Rivard et al., 2004b) dose calculation methodology. In contrast to the TG-43U1 methodology where, for dose calculation purposes, the patient is treated as a $15 \mathrm{~cm}$ radius sphere of water with uniform unity density, TG-186 recommends the use of model-based dose calculation algorithms (MBDCA) alongside current TG-43U1 methods for dosimetry. While TG-186 has relatively minor consequences for dose calculation in high energy ${ }^{192} \mathrm{Ir}$ brachytherapy, these updated guidelines have a greater impact for low-energy brachytherapy where the dose calculations are known to depend strongly on the composition of the interacting tissues(Landry et al., 2010b) as well as tissue heterogeneities(Afsharpour et al., 2011a; Sutherland et al., 2012).

Of particular relevance is the application of TG-186 to photon emitting sources found in Accelerated Partial Breast Irradiation (APBI) particularly low energy eBx sources such as the Zeiss Intrabeam (Carl Zeiss, Oberkochen, Germany) and the Axxent (Xoft, Fremont, CA). One of these systems, the Axxent, is similar to the MammoSite system (Hologic Inc., Bedford, MA) in which a balloon catheter is inserted into a breast lumpectomy cavity. The MammoSite system uses an ${ }^{192} \mathrm{Ir}$ source whereas the Axxent uses a $50 \mathrm{kV}$ x-ray eBx source. The Axxent source is a miniature x-ray tube contained within a $5.1 \mathrm{~mm}$ flexible cooling sheath. Water is continuously circulated throughout the source's activation to prevent overheating. The source is non-radioactive, making it easier to store and transport. The dose rates at $1 \mathrm{~cm}$ along the transverse axis in water are equivalent to a $370 \mathrm{GBq}$ (10ci) HDR ${ }^{192} \mathrm{Ir}$ source while the depth-dose characteristics are similar to ${ }^{125} \mathrm{I}$ and ${ }^{103} \mathrm{Pd}$ sources(Rivard et al., 2006b). The Axxent source can be operated at a variety of voltages but is clinically mainly used at $50 \mathrm{kV}$. By comparison to ${ }^{192} \mathrm{Ir}$, which emits photons of average energy $380 \mathrm{keV}$, the Axxent has a low energy source that emits photons as a continuous spectrum up to $50 \mathrm{keV}$ with an average energy of $33 \mathrm{keV}$ (Liu et al., 2008). This allows the operation of the device with minimal shielding needed for medical staff during treatment. Low energy photons interact with tissue partially via the photoelectric effect which has an interaction probability that depends strongly on the average atomic number $(Z)$ of the tissue $\left(\sim Z^{3-4}\right)$. Knowledge of the composition/type of the breast tissue to be irradiated is therefore very important.

Tissues in the breast are composed of glandular and adipose tissues (Figure 1). The breast is structured with complex tree-like high density glandular tissue, composed of ducts and lobules, embedded in adipose confined on two sides by skin and ribs(Nelson et al., 2008). TG-186 recommends tissue/material assignment based on contours approved by the radiation oncologist. However adipose and glandular tissue in the entire breast is not contoured. The proportion of glandular to adipose tissue varies in the population(Yaffe et al., 2009) which 
has been shown to be a significant factor in dose calculations for low energy sources like

${ }^{103} \mathrm{Pd}$ (Afsharpour et al., 2010) or ${ }^{125} \mathrm{I}$ (Furstoss et al., 2009). Moreover, the tissue composition of individual patients is not accurately known; the compositions listed in reports such as ICRU 46(International Commission on Radiation Units and Measurements, 1992) and ICRP 89(International Commission on Radiological Protection, 2002) are population averages based on samples. It has been shown that dose to specific tissues of interest in segmented breast models can show significant differences to breast models using composite breast compositions(Afsharpour et al., 2010; Sutherland et al., 2011).

In our study we present a retrospective TG-186 model-based dose calculation on patients who underwent APBI with the Axxent eBx source. We assessed the influence of using a realistic multi-tissue patient geometry (heterogeneous model) to determine the dose distribution. Two different tissue modeling schemes were explored. The importance of proper balloon applicator modeling was also studied. We compared dose distributions calculated using TG43, two TG-186 models and two dose scoring methods.

\section{Methods and Materials}

\section{Patient Cases}

3D treatment planning CT images from 7 patients who underwent APBI were used to create the multi-tissue patient geometries simulated in this study. The CT image data was anonymized and de-identified prior to entry in this study. All patients had the Xoft balloon applicator inserted at the time of CT imaging and each patient was treated with the Axxent S700 source. The BrachyVision (Varian, Palo Alto, CA) treatment planning system (TPS) was used for determining the TG-43 dose calculations. The prescription dose regime for each patient was 10 fractions of 3.4 Gy for a total dose of 34 Gy. All treatment plans used a single applicator with multiple dwell positions and dwell times. Delineations of the planning treatment volume (PTV), skin and balloon wall were provided. The PTV margins were 0.8 $\mathrm{cm}$ to $1 \mathrm{~cm}$ for all 7 patients. The minimum PTV edge-to-skin distance varied from $0 \mathrm{~cm}$ to $1.7 \mathrm{~cm}$ and the breast size ranged from $340 \mathrm{~cm}^{3}$ to $2000 \mathrm{~cm}^{3}$. The CT data set provided the $\mathrm{MC}$ simulation under a variety of different backscatter conditions and tissue structures. The air kerma source strength $\left(\mathrm{S}_{\mathrm{k}}\right)$, dwell times and positions were obtained from the DICOM file generated by the TPS. 


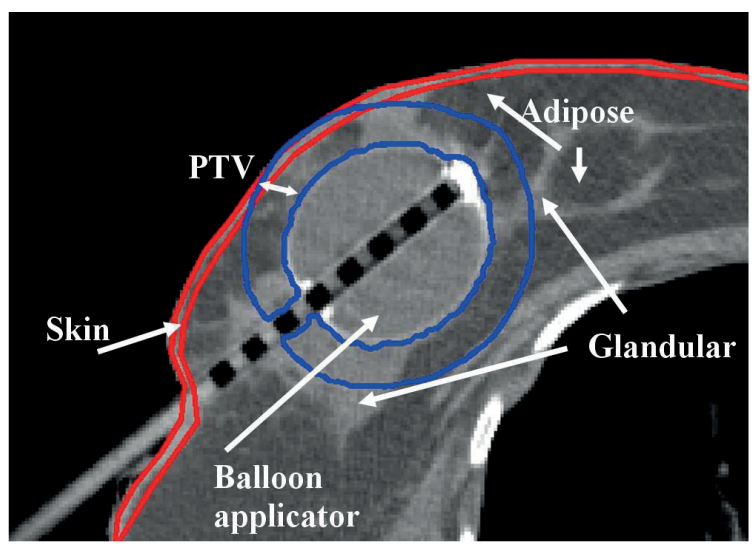

Figure 1: A CT slice of an APBI patient highlighting the PTV (inside blue contours) and skin contour (red). The PTV is contoured around the balloon applicator while the source is inserted into the hollow air channel in the centre of the balloon during treatment. Adipose and glandular tissues are highlighted.

\section{Geant4, patient geometry generator and dose analysis tool}

The software package used for the simulations in this study was the Monte Carlo (MC) toolkit Geant4 v9.5p02. Geant4 is an all purpose MC simulation toolkit that provides a diverse set of software components that can be employed in a variety of settings, including the simulation of photon transport from low-energy sources(Agostinelli et al., 2003b). This paper used the Lawrence Livermore National Laboratory low-energy electromagnetic model which simulates the low-energy photon interactions of the photoelectric effect, Compton scattering and Rayleigh scattering. The model uses the EPDL-evaluated(Cullen D, 1997) and EADL-evaluated(Perkins, 1997) libraries. All secondary electrons were made to deposit their energy locally because of their short range compared to typical voxel sizes; hence the dose was approximated by collision kerma. The photon energy transport cutoff was $1 \mathrm{keV}$. In accordance with the TG-186 recommendations, both dose to medium in medium $\left(\mathrm{D}_{\mathrm{m}, \mathrm{m}}\right)$ and dose to water in medium $\left(D_{w, m}\right)$ were reported along with dose to water in water $\left(D_{w, w}\right)$ from TG-43U1. In this notation, the first index refers to the dose-scoring medium ( $\mathrm{m}$ or $\mathrm{w}$ ) and the second index indicates the photon transport medium ( $m$ or w). $D_{w, w}, D_{w, m}$ and $D_{m, m}$ were all scored using track-length scoring(Williamson, 1987) with mass energy-absorption coefficients lookup tables used. All calculations were performed using a networked cluster of computers using HTCondor(Douglas, 2005) to reduce calculation time.

CT DICOM images were used to generate Geant4-compatible patient geometries. The Hounsfield unit to density conversion was calibrated against CT quality assurance scans performed using the CatPhan phantom (The Phantom Laboratory, Salem NY). Certain inserts were excluded from the calibration to ensure a linear fit to enable better distinction of adipose and glandular tissue. The conversion of these images to Geant4-compatible geometries was performed using BrachyGUI(Poon et al., 2008a), an in-house dose analysis tool. BrachyGUI utilizes the CT Hounsfield units and assigns a material and a density to each voxel in a format to create an egsphant file(Kawrakow, 2007) (Figure 2). The assignment of each material can be density-based (if the materials density range is known) or manually changed (if contour information is available). The voxel resolution of the DICOM images in this study, ranging 
from $0.42 \mathrm{~mm} \times 0.42 \mathrm{~mm} \times 3 \mathrm{~mm}$ to $0.97 \times 0.97 \times 3 \mathrm{~mm}$, was maintained through the conversion. BrachyGUI also functions as a dose analysis tool. It was used to compare dose metrics between the dose distributions from different patient models. Dose ratio maps were also used to visualize hot/cold dose regions when comparing distributions directly.

\section{Source modeling}

The Axxent source (hereafter referred to as the S700) was modeled in Geant4(Liu et al., 2008) based on detailed descriptions and diagrams of the sources' tip and validated against MC simulations described in Rivard et al.(Rivard et al., 2006b). The exact compositions and dimensions of the S700 source are proprietary. In this model, $50 \mathrm{keV}$ electrons are set in motion at the cathode and transported to a tungsten anode. Photons were generated via bremsstrahlung splitting $(\mathrm{N}=100)$ and characteristic $\mathrm{x}$-ray generation. Photons that reach the outer cooling water sheath of the source (diameter $5.1 \mathrm{~mm}$ ) were recorded into a phase space file and then terminated. $10^{9}$ primary electrons were simulated to generate a phase space file with $7 \times 10^{7}$ photons. No electrons were recorded due to their short range. The phase space source was validated against TG-43 data from Rivard et al(Rivard et al., 2006b). to within tolerated uncertainties $(<3 \%)$. This phase space source was used to simulate photon emission from the S700 at several dwell positions for each patient. It should be noted that S700 source used in experiments described later ( see section Tip/Air channel modeling) has a slightly different tip design compared to the older MC model in this study and Rivard et al.(Rivard et $a l ., 2006 b)$. The impact of the updated design has not been explored.

\section{Absolute MC dosimetry}

The phase space source was used to sample photons from a source model to simulate a relative dose distribution. For a dosimetry evaluation study, absolute dose is calculated. A conversion factor for Geant 4 dose per particle values to absolute dose values was needed to properly compare TPS-based plans to Geant4-based plans. The conversion from dose per simulated photon to absolute dose was based on air kerma measurements performed on an Attix free-air chamber described in a separate study(Davis, 2009). A MC simulation, based on the Attix chamber experiment(Davis, 2009), was performed where a small scoring volume, modeled as the air chamber with a $1 \mathrm{~cm}$ diameter aperture with no cover/filter, was placed at 1 meter from the source in an air phantom at STP. The contribution of external backscatter was negligible in the measured values and was not accounted for in the simulation. The MC relative air-kerma was calculated from the simulated air chamber and used in conjunction with the measured air kerma (uncorrected for air attenuation) to create an absolute dose conversion factor for Geant4. As a validation, the dose rate constant (DRC) was also calculated $(0.69 \mathrm{cGy} / \mathrm{hr} / \mathrm{U})$ and compared against the measured value $(0.709 \mathrm{cGy} / \mathrm{hr} / \mathrm{U})(\mathrm{Xoft}, 2009)$ with good agreement $(\sim 3 \%)$. The dose per particle to real dose conversion factor was applied to all simulation results based on the treatment plan's reference $\mathrm{S}_{\mathrm{k}}$. Due to the lack of correction for air attenuation and backscatter, care should be taken not to confuse these $S_{k}, D R C$ and $U$ in air values with real in-vacuo values used in other brachytherapy calibration standards. The absence of backscatter and air attenuation correction 
factors reflect the current air kerma calibration method provided by Xoft. Future updates to the S700 air kerma calibration may include these corrections. It should be noted that absolute dose (Gy), instead of relative dose (arbitrary units), was calculated for all patient geometry simulations for the purposes of future clinical studies with the Axxent eBx device. However, as this is an intra-comparison Geant 4 study, absolute dose is not essential to draw conclusions from this study's results.

\section{TG-43 Validation}

To validate the Geant4 TG-186 patient calculations, a Monte Carlo-based TG-43 (TG-43 $\mathrm{MC}$ ) simulation was performed on all of the patient geometries. During the conversion from DICOM to egsphant in BrachyGUI, all voxels were assigned as water with unity density and all were simulated according to the original TPS reference $S_{k}$, dwell positions and dwell times with the S700 phase-space file, described in previous sections. All regions outside of the egsphant volume were set to water with unity density to simulate TG-43-equivalent backscatter. A comparison of the TG-43 MC results for each patient was made with the original treatment plan from the BrachyVision TPS. A visual comparison was made using BrachyGUI which allowed the use of dose ratio maps. The dose metric $\mathrm{D}_{90}$, the minimum dose to $90 \%$ of the PTV volume, the percentage of the PTV receiving $100 \% 150 \%$ and $200 \%$ of the prescribed dose, $\mathrm{V}_{100}, \mathrm{~V}_{150}$ and $\mathrm{V}_{200}$ were calculated for each of the patients in both the TPS plan and the MC result and used to compare both calculation methods.

\section{Water-Air model}

As an intermediate to the heterogeneous model, a Water-Air model for each patient was generated and simulated. All tissue (density $>0.26 \mathrm{~g} / \mathrm{cm}^{3}$ ) and applicator voxels were assigned as water with unity density while low density tissue (density $<0.26 \mathrm{~g} / \mathrm{cm}^{3}$ ) such as lung and areas outside of the body were assigned as air (density $=0.0012 \mathrm{~g} / \mathrm{cm}^{3}$ ). This simple model was similar to TG-43 but took into account the absence of backscatter from air outside the patient (which contributes to the overestimation of skin dose when using TG-43)(Raffi et $a l ., 2010)$ and low-density lung tissue inside the patient. This would be used to isolate the effect of tissue heterogeneity in regions where non-patient backscatter (or absence of) would be an important factor in dose differences when comparing to TG-43. The individual contribution of each of the backscatter components, lung backscatter and non-patient backscatter, was also calculated by selectively assigning each region to air or water.

\section{Heterogeneous model}

A fully segmented tissue model was the basis for the realistic multi-tissue patient geometry. The segmented model (referred to as the heterogeneous model hereafter) is a realistic patient geometry using CT-derived densities and proper tissue assignment and composition. This model is the recommended tissue model for TG-186 dose calculation(Beaulieu et al., 2012). For simplicity, only 5 tissues were modeled, based on their clinical relevance: skin, adipose tissue, glandular tissue, lung and rib. Muscle tissue was not modeled. The assignment of air, lung, rib or skin to voxels was based on their density and/or anatomical location. Adipose and 
glandular tissues were more difficult to distinguish using density assignment as there exists a degree of overlap between the two in terms of density(Afsharpour et al., 2010). A threshold between the two was calculated, first, by averaging multiple density samples of regions of breast which were unambiguously adipose or glandular tissue. This established the approximate density range for both tissues. The final threshold was chosen as the minimum density at which the glandular duct structure most accurately reflected that on the CT image (minimum $0.94 \mathrm{~g} / \mathrm{cm}^{3}$ ). Each patient had a different threshold, perhaps due to age-related density changes though the ages of the patients were unknown. Due to the nature of the threshold and the overlapping density ranges of adipose and glandular tissue, some adipose was modeled as gland. The composition for these tissues was taken from ICRU 46(International Commission on Radiation Units and Measurements, 1992). (Table 1). The tissue segmentation was visually inspected for all patients (Figure 2).
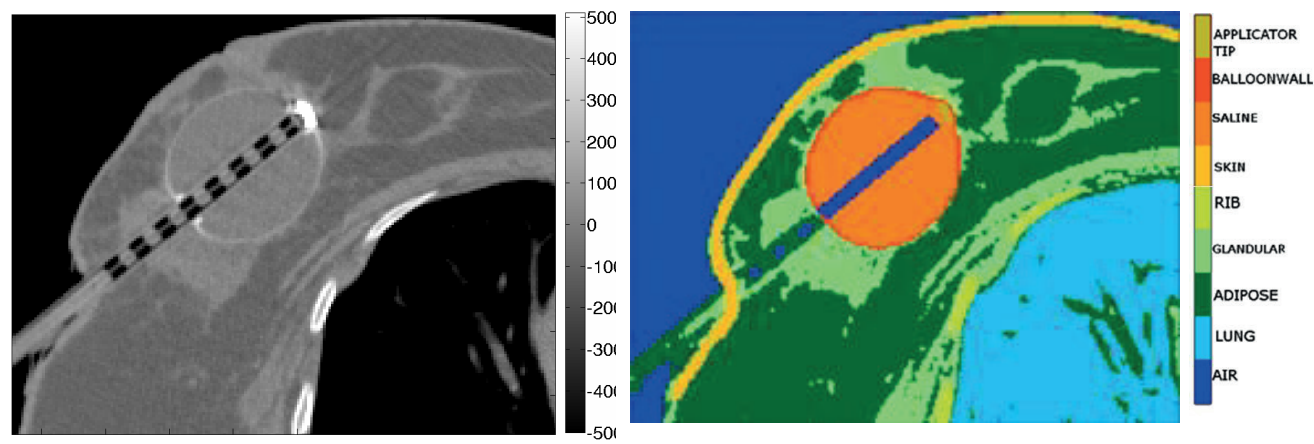

Figure 2: An egsphant geometry file for Geant4 simulation with a fully-segmented breast geometry (right) converted from a HU-based CT image (left). The balloon applicator is also modeled here to highlight the air channel, saline-filled balloon and balloon tip (appearing as an artifact due to high absorption of barium).

The balloon applicator was modeled using 5 materials, air, saline solution, and 3 proprietary applicator materials. Air was present in the hollow applicator channel where the S700 source is inserted and repositioned at dwell points inside the applicator during the treatment. The applicator balloon is filled with a $0.9 \%$ weight per volume saline solution during APBI treatment. The applicator materials are the balloon wall, which is composed of bariumsulphate silicone compound, (acting as contrast medium for visibility on CT images), a balloon tip which compensates for the lack of attenuation by the air channel, prominent when dwell positions are far away from the tip of the applicator and a stiffener which maintains the rigidity and shape of the air channel (Figure 3). 


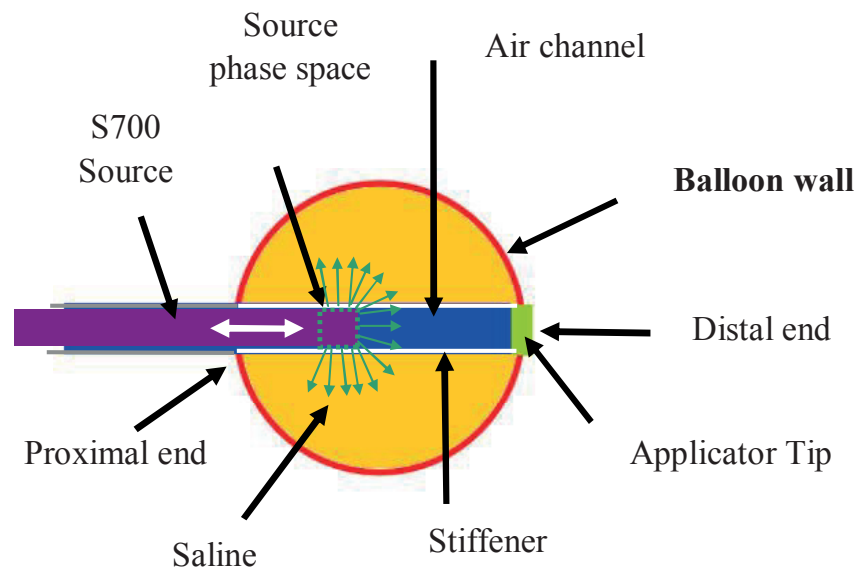

Figure 3: A schematic diagram of the Axxent balloon applicator. The S700 source (purple) can be placed at multiple dwell positions inside the applicator to deliver the desired dose distribution, creating an air channel of variable length (blue). The source is replaced by a phase space file inside the applicator (green arrows) during MC simulation.

Table 1: The ICRU 46 tissue compositions for the 5 tissues modeled in the patients

\begin{tabular}{l|lllllllllll}
\hline \hline \multicolumn{1}{c}{ Element (\% weight) } \\
\hline Tissue & H & C & N & O & Na & Mg & P & S & Cl & K & Ca \\
\hline Glandular & 10.6 & 33.2 & 3 & 52.7 & 0.1 & - & 0.1 & 0.2 & 0.1 & - & - \\
Adipose & 11.4 & 59.8 & 0.7 & 27.8 & 0.1 & - & - & 0.1 & 0.1 & - & - \\
Skin & 10 & 20.4 & 4.2 & 64.5 & 0.2 & - & 0.1 & 0.2 & 0.3 & 0.1 & - \\
Rib & 6.4 & 26.3 & 3.9 & 43.6 & 0.1 & 0.1 & 6 & 0.3 & 0.1 & 0.1 & 13.1 \\
Lung & 10.3 & 10.5 & 3.1 & 74.9 & 0.2 & - & 0.2 & 0.3 & 0.3 & 0.2 & - \\
\hline \hline
\end{tabular}

\section{Balloon Applicator modeling}

\section{Balloon wall}

The balloon wall consists of a $0.3-0.5 \mathrm{~mm}$ thick silicone shell with a relatively high concentration of barium sulphate (figure 3). The barium also attenuates the photon fluence emitted from the source during treatment to a greater degree than an equal thickness of water(Segala et al., 2011). The decrease in dose due to balloon wall attenuation in treatments is not accounted for by TG-43 dosimetry but the manufacturer recommends an increase of $6 \%$ in source dwell times to offset the attenuation. Previous studies(Segala et al., 2011) had shown that the degree of attenuation by the balloon was not uniform due to the variable thickness of balloon wall material that photons will traverse depending on its angle of incidence. This could not be corrected for by increasing the dwell times uniformly. The tip composition of the balloon applicator varied from patient to patient due to the type of applicator used and the degree to which it was inflated and deformed by the surrounding tissue. The balloon wall was difficult to model using the same CT to egsphant geometry approach as used for the tissues since the thickness of the wall $(\sim 0.4 \mathrm{~mm})$ is much smaller than standard voxel sizes seen in the CT images of patients (up to $3 \mathrm{~mm}$ ). Thus voxelization 
of the balloon wall leads to an over-estimation of the attenuation and consequently an underestimation of the dose to the surrounding tissue.

Methods of modeling structures in voxelized patient geometries have been described in a previous study(Enger et al., 2012). Geant4 allows the creation of parallel world geometries(Apostolakis, 2008) which can be used to overlap existing voxel geometries. Normally these geometries contain no mass and do not have any influence on the physical interactions of particles. A technique using Layered Mass Geometries (LMG) allows the analytic modeling of simple geometries, such as ${ }^{125}$ I seeds, which can influence the transport of particles throughout the patient geometry. While LMG can be used to define simple objects, more complex geometries, such as a patient deformed balloon applicator, can not be recreated in the parallel world easily using combinations of simple geometries. Other sections of the applicator could be modeled by simple geometries and are referred to in the following section. A more complicated approach was needed for the balloon wall.

First, balloon wall contours for each patient, contained within the DICOM data, were imported into Meshlab (ISTI-CSI, Rome), an open source mesh-generating/processing tool. Meshlab links the balloon wall contour points to one another based on their nearest neighbour to construct a series of tetrahedral shapes that link together to create a convex hull mesh. A convex hull is the minimum geometrical volume that contains all of the contour points such that the vector between any two of the contour points does not lie outside of the volume. This convex hull was the outer surface of the balloon. As the balloon wall is a shell-type structure, a second geometry was needed to define the inner surface. A second convex hull geometry was made from the contour points and scaled down in size (using the balloon's center of mass as reference) so that each point of the surface was $0.4 \mathrm{~mm}$ from its original position. This corresponds to the average thickness of the balloon wall over the entire applicator. Each of these geometries was saved separately as an inner and outer surface mesh files. The combination of both of these meshes was used to create the balloon wall.

CADMesh is a $\mathrm{C}++$ library for loading CAD based geometries into Geant4(Poole et al., 2012). The conversion of the mesh balloon wall geometry into a CADmesh-compatible assembly was performed using Tetnest(Poole et al., 2012), a python-based script that imports surface meshes and creates the nested tetrahedral mesh. Figure 4 show how a uniformly thick $(0.4 \mathrm{~mm})$ balloon wall model was created for the heterogeneous model simulations using Meshlab and Tetnest. 

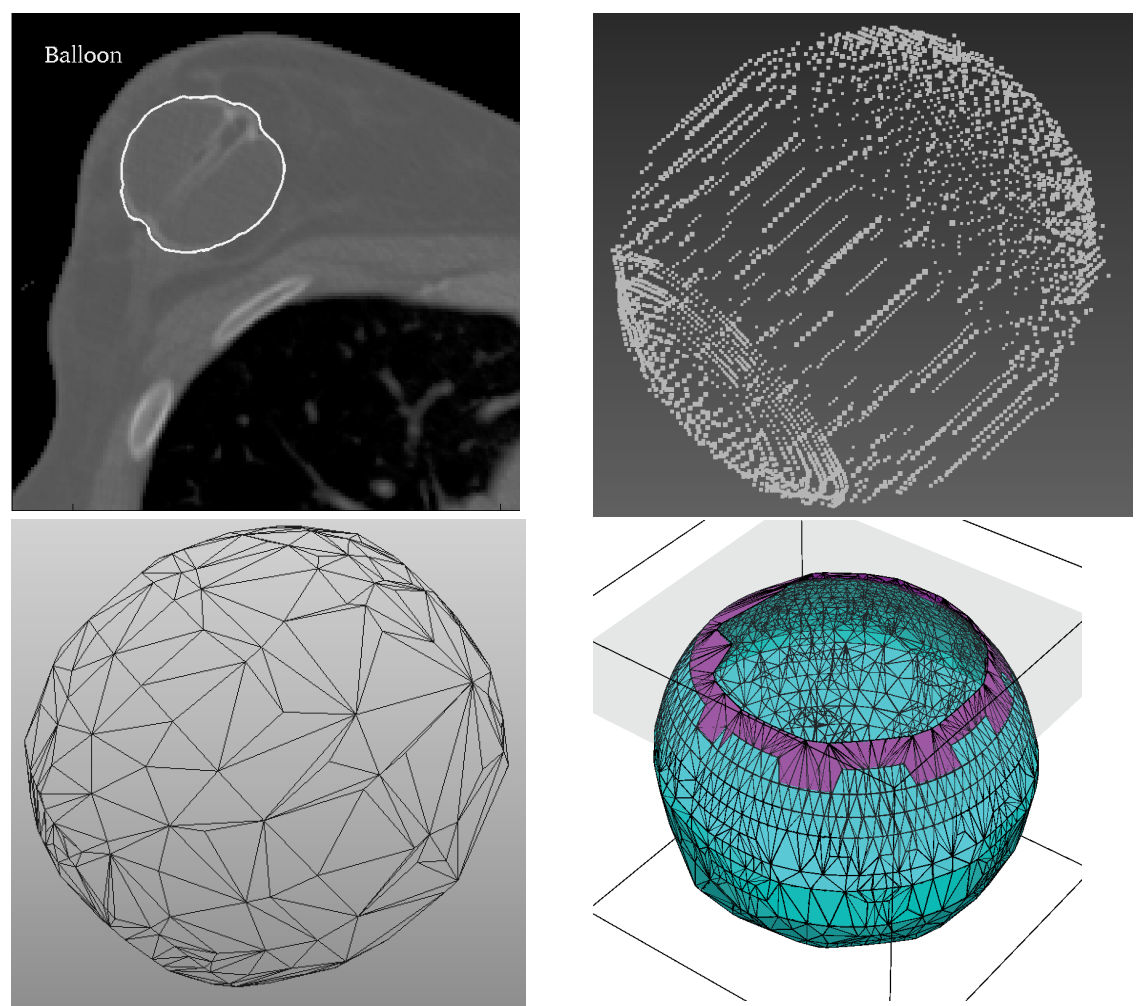

Figure 4: The conversion of the irregularly shaped balloon wall from a DICOM RT structure (Top left and right) to a complex array of tetrahedrals that create a convex hull geometry (Lower left) calculated using Meshlab and finally the CADmesh balloon wall (Lower right) created in Tetnest. The CADmesh wall geometry has been cut in the z-plane to display both surfaces. The green denotes the outer surface and the pink, the inner.

The balloon wall material was assigned to the geometry. The balloon wall geometry for each patient was created separately, based on the existing balloon applicator TPS contours. This procedure could be useful for many other applications with complex geometries. The technical aspects of this procedure and some benchmarks will be detailed in a future publication.

\section{Tip/Air channel modeling}

The air channel and tip of the balloon applicator were modeled using the LMG technique. Given the high concentration of high- $Z$ material in the applicator tip, its irregular shape and small dimensions, voxelization of the applicator led to an over-estimation of its dimensions and an over-attenuation of dose in the region around the tip. Voxelization of the air channel results in an over/under estimation of the diameter of the air channel due to the large slice thickness $(3 \mathrm{~mm})$ of the CT image depending on the angle and orientation of the applicator. Thus the tip and air channel were modeled using a combination of analytical geometries as much as possible. The dimensions of the air channel and the applicator tip were provided by Xoft. The resultant geometries were placed in the same parallel world as the CADmesh 
balloon wall. The saline solution inside the balloon was the only section of the applicator that was voxelized. The composition of the tip varied depending on the size/type of the applicator used, which varied from patient to patient.

\section{CADmesh Validation}

Validation of the mesh/LMG technique was performed in Monte Carlo using a simple spherical geometry with a small finite wall thickness as a benchmark geometry. The geometry consists of a $0.4 \mathrm{~mm}$ thick wall that encloses a water sphere of radius $25 \mathrm{~mm}$. Like the balloon applicator, this wall geometry was constructed from two concentric spherical clouds of points which were changed into surface meshes in Meshlab and converted into a CADmesh compatible format by Tetnest, as described in the previous section. This wall geometry was placed in a $10 \mathrm{~cm} \times 10 \mathrm{~cm} \times 10 \mathrm{~cm}$ voxelized water world (voxel size $1 \mathrm{~mm}^{3}$ ). The geometry was simulated in Geant 4 using a $50 \mathrm{keV}$ mono-energetic point source placed at the centre of the sphere. An identical geometry was simulated using analytical geometry modeling techniques in Geant 4 and compared against the CADmesh dose calculations. $2 \mathrm{x}$ $10^{9}$ histories were simulated for both cases and the maximum statistical uncertainty, at the outermost voxel was $<1 \%$. Comparisons between the results were made using dose-ratio maps and all voxels agreed to within $<0.5 \%$.

\section{Experimental validation}

Validation of the mesh/LMG technique and the tip/air channel modeling was performed experimentally using EBT3 GAFchromic film using a simple in-house phantom. A water inflated Axxent balloon applicator was immersed in a PMMA box phantom approximately $6 \mathrm{~cm} \times 6 \mathrm{~cm} \times 6 \mathrm{~cm}$ in dimensions with the remaining space inside the box filed with water. Pieces of EBT3 film were placed on three sides of the box and surrounded by PMMA backscatter (Figure 5). All film was irradiated to a minimum of $0.5 \mathrm{~Gy}$ and a maximum of 8 Gy. The EBT3 film was scanned using an EPSON PRO v750 scanner at 225dpi and processed using the triple channel method using Matlab(Hoof et al., 2012).

A $1 \mathrm{~mm} \times 1 \mathrm{~mm} \times 0.6 \mathrm{~mm} 120 \mathrm{kVp}$ CT scan of the phantom was used to create an egsphant geometry for allowing MC simulation of the experiment setup An LMG balloon wall was created using contours of the applicator obtained from the CT scan. The LMG balloon wall was included as a parallel world geometry using the method described in section.2.8.1. Relative comparisons between the experimental and normalized MC results were evaluated using line profiles.

\section{Inter-model comparison}

DVH metrics were used to compare various dose-distributions quantitatively. The $\mathrm{D}_{90}$ (minimum dose received by at least $90 \%$ of the volume of the PTV) was expressed as a percentage of the fractional dose of $3.4 \mathrm{~Gy}$. The volume metrics were expressed as a percentage of the total PTV volume. The volume metrics are important in terms of toxicity as $\mathrm{V}_{150}$ and $\mathrm{V}_{200}$ (volume receiving at least $150 \%$ or $200 \%$ of the prescribed dose) in interstitial brachytherapy have been correlated with increased occurrence of fat necrosis(Budrukkar et al., 2012; Wazer et al., 2001). Any differences between PTV metrics of different models were expressed as an absolute percentage and not a renormalized value. The doses to separate individual tissues in the PTV were not evaluated. The dose to skin was evaluated as the 
highest minimum dose at any $0.2 \mathrm{cc}$ region of the skin $\left(\mathrm{D}_{0.2 \mathrm{cc}}\right) \cdot \mathrm{D}_{0.2 \mathrm{cc}}$ was expressed as a percentage of the prescription dose. In addition to dose metrics, dose ratio maps comparing each plan were calculated using BrachyGUI which calculated a ratio based on a direct voxel by voxel comparison between plans. Dose ratios are shown for $D_{m, m}$ and $D_{w, m}$ for the heterogeneous model vs. TG-43 MC for each patient. As dose to voxels outside the body are clinically irrelevant, dose to these voxels were not scored hence the dose in these sections is zero and should be ignored. A dose ratio map was also calculated for the heterogeneous model versus the Water-Air model. This dose ratio map isolated the dosimetric impact due to tissue heterogeneities alone.

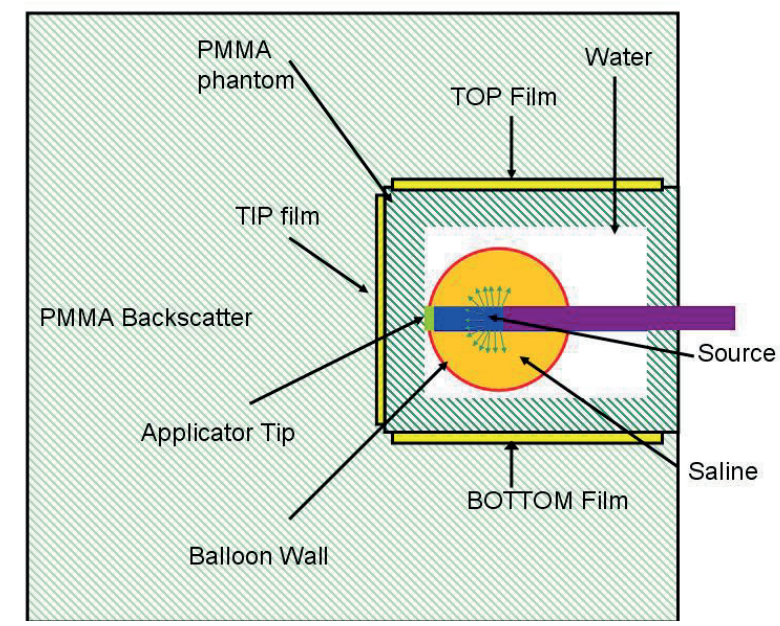

Figure 5: Schematic diagram of experimental setup for the CADmesh validation

\section{Results and Discussion}

\section{TG-43 Validation}

When the TG-43 MC was validated against the BrachyVision TPS, the $\mathrm{D}_{90}$ agreed to within an average of $2.8 \%(\sigma=0.2 \%)$ (Table 2$)$ for the 7 patients. Other dose metrics agreed to within $2-3 \%$. The dose ratio of the two dose maps revealed that the largest discrepancies (10$20 \%$ ) existed inside the balloon, close to the applicator channel and along the applicator proximal end $\left(160^{\circ}\right.$ to $\left.175^{\circ}\right)$. It should be noted that disagreement between calculated and measured doses in these regions around the source has been reported previously(Rivard et al., 2006b). These regions are not clinically significant due to their location. A reason for this disagreement could be due to the way the TPS interpolates the dose close to the center. The systematic discrepancy could be due to the difference in MC code used for the Rivard et al. validation dataset (version MCNP5) and that used in this study (Geant4). Small differences in the source model may also pay a role as uncertainty in the position of the phase space origin and the thickness of the anode can have a large impact on the dose distribution. 
Table 2: Average percentage difference between BrachyVision (TPS) and TG-43 MC calculation methods for dose metrics for the PTV for 7 patients. $\mathrm{D}_{90}$ and its standard deviation $(\sigma)$ are expressed as a percentage of the prescription dose.

\begin{tabular}{c|cccc}
\hline & \multicolumn{4}{|c}{ Metric } \\
Model & D90 (\%) & V100 (\%) & V150 (\%) & V200 (\%) \\
\hline TG-43 TPS (average) & 101.2 & 90.9 & 46.3 & 21.3 \\
TG-43 MC (average) & 103.9 & 93 & 49.1 & 23.5 \\
TPS - TG-43 MC & 2.8 & 2.1 & 2.8 & 2.2 \\
$\boldsymbol{\sigma}$ & 0.2 & 0.4 & 0.2 & 0.3 \\
\hline \hline
\end{tabular}

\section{Experimental validation}

Line profiles for the top and bottom film pieces versus the Monte Carlo showed good agreement to within experimental/MC uncertainty $(0.5-2.0 \%)$ (Figure $6 \mathrm{a})$. The MC results were normalized to the max dose on each film piece for the TOP and BOTTOM region by the same scale. The difference between the top and bottom line profile can be explained due to the asymmetry of the phantom, as the source was not at equal distance from both film pieces. The TOP region (Fig 5) was also influenced by air bubbles within the applicator balloon which were accounted for in the MC model. Some disagreement was observed with the TIP region (figure $6 \mathrm{~b}$ ) where the $\mathrm{MC}$ model underestimates the peak dose around the central region (where the film was placed directly above the applicator tip) by between $6-20 \%$. The TIP max dose was normalized to the MC by same normalization value as the TOP and BOTTOM pieces. The TIP disagreement between the MC and film model can be attributed to source position uncertainties which are enhanced by the air channel in the applicator. The uncertainties in the analytical geometry of the applicator tip were also a significant factor as were the uncertainties and variation in the balloon wall thickness particularly at the front. Despite this, the analytical/CADmesh applicator geometry is an improvement over voxelized models of the applicator which can overestimate the wall thickness by a factor of 4 . 
(a) Line profile: Film vs. Monte Carlo, TOP and BOTTOM Region

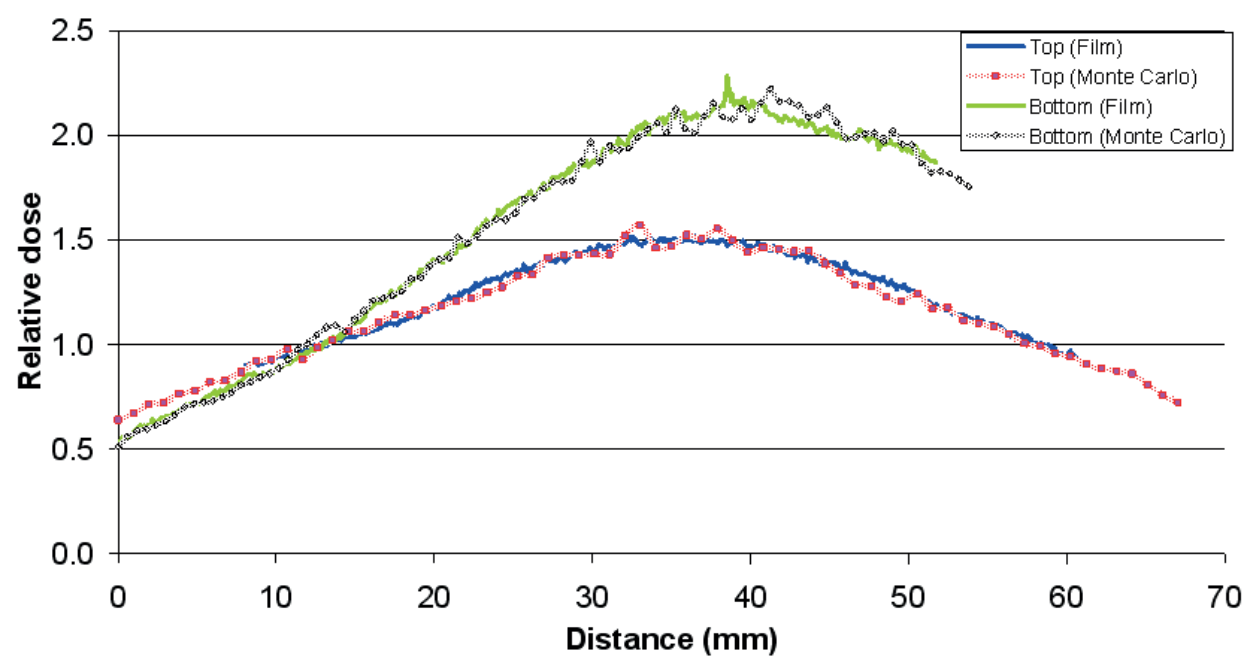

(b) Line Profile: Film Vs Monte Carlo, FRONT region

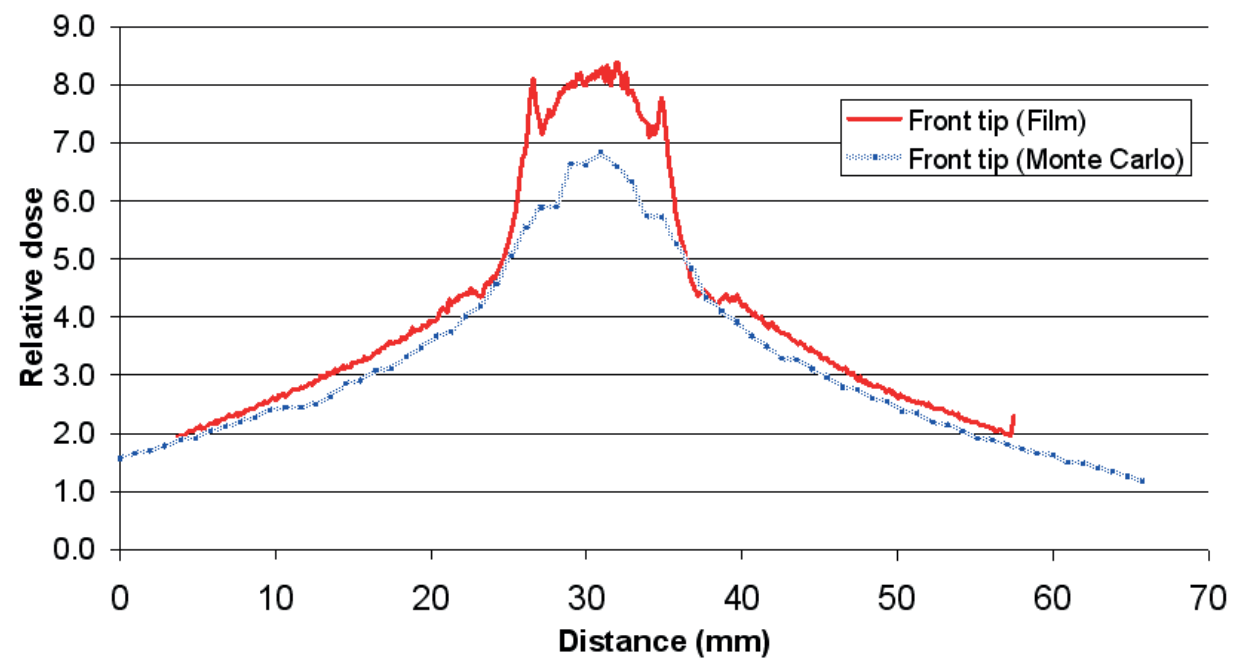

Figure 6: Line profiles of each of the film pieces from various areas of the phantoms versus the line profiles from equivalent regions on MC model. The TOP and BOTTOM regions are highlighted in (a) and the FRONT region in (b).

\section{Water-Air model vs. TG-43 MC}

The dose ratio map for the Water-Air model / TG-43 MC model can be seen in figure 7a. The ratio map demonstrates the regions where the absence of backscatter in the Water-Air model has the largest impact, most notably the skin. The DVH differences between both models (Figure 7b) show the effects of substituting an all-water geometry for a more geometrically 
realistic water model taking the breast shape, the air and the presence of lungs into account. For 7 patients, an average $\mathrm{D}_{90}$ reduction of $3.8 \%(\sigma=2.4 \%)$ (Table 3$)$ was calculated for the Water-Air model. Most of the reduction could be attributed to the absence of non-patient backscatter $(\sim 3.2 \%)$. The absence of backscatter in the lung accounted for the remainder ( $0.6 \%$ ) (Figure 7c) making it approximately $20 \%$ of the non-patient backscatter though the range of the contribution of the lung backscatter varied from $0 \%$ (for large breasts with PTV closer to the skin) to $37 \%$ (for small breasts or the PTV close to the ribs). The skin dose, $\mathrm{D}_{0.2 \mathrm{cc}}$ was much lower for all patients with an approximate mean decrease of $9.4 \%$ from the TG-43 $\mathrm{D}_{0.2 \mathrm{cc}}$ (Range $-4.9 \%$ to $-14.3 \%$ ) in the Water-Air model signifying the large portion of dose contributed by erroneously modeled non-patient backscatter in TG-43. Lung backscatter constituted almost zero skin dose. Patient geometry, applicator positioning and dwell times play a large role in determining the dose reduction in the PTV, reflected in the relatively large range of $D_{0.2 c c}$ reduction of between $0.7 \%$ to $7.2 \%$. Larger breasts and deeper seated applicators lessen the impact of reduced backscatter from the outside air as is evident from the minimum PTV to skin distance measured in the Water-Air models which ranged from $0 \mathrm{~cm}$ to $1.7 \mathrm{~cm}$ for a PTV dose reduction of $7.2 \%$ and $0.7 \%$, respectively. High skin dose is more probable in smaller breasts thus proper backscatter modeling is important. Skin dose has been shown to be critical to predicting cosmetic outcome of ${ }^{192}$ Ir-based APBI(Vargo et al., 2014a), though the dosimetry in this study was performed using TG-43. While there is an impact of backscatter on the skin dose for the low energy dosimetry, it is likely to be more important for higher energy dosimetry $\left({ }^{192} \mathrm{Ir}\right.$ and $\left.{ }^{137} \mathrm{Cs}\right)$ as a previous study has shown(PerezCalatayud et al., 2004) due to greater Compton scattering cross-section at higher energies.

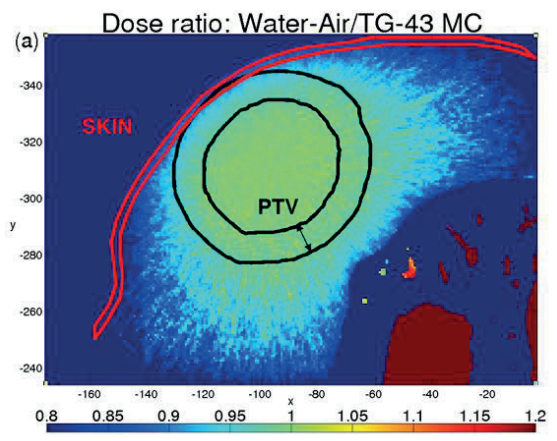

(c) Dose and volume metric reduction due

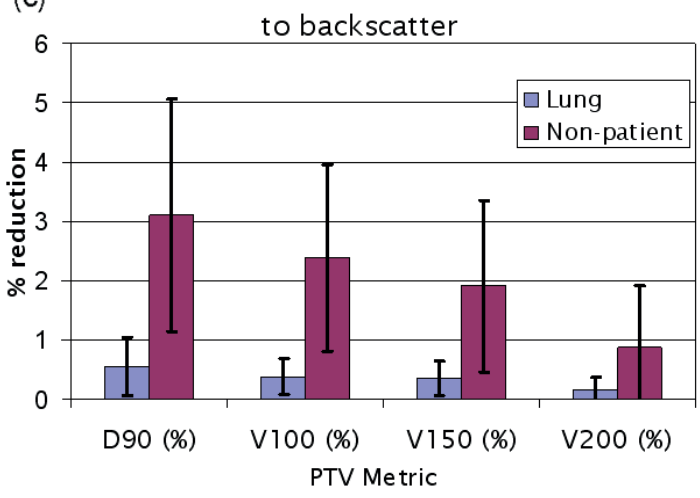

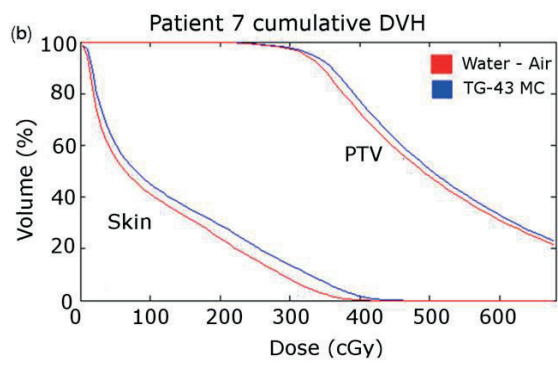

Figure 7: (a) Dose ratio map for patient 7; WaterAir model / TG-43 MC. For the Water-Air model both lung and non-patient sections are modeled as air. Dose to air is zero in Water-Air. (b) A comparative DVH distribution for patient 7 taking into account the skin and PTV contours. (c) The contribution of lung and non-patient backscatter in TG-43. 


\section{Heterogeneous model vs. TG-43}

Figures $8 \mathrm{a}$ and $\mathrm{b}$ shows the dose ratio maps of the heterogeneous model / TG-43 MC with the dose scored as $D_{w, m}$ (figure $8 \mathrm{a}$ ) and $\mathrm{D}_{\mathrm{m}, \mathrm{m}}$ (figure $8 \mathrm{~b}$ ). The figure also shows the dose ratios of the heterogeneous model / Water-Air model for $D_{w, m}$ (figure $8 c$ ) and $D_{m, m}$ (figure $8 d$ ). The slice of the simulated heterogeneous model's egsphant geometry, from which the dose ratio maps were obtained, can be seen in figure $8 \mathrm{e}$. In figure $8 \mathrm{a}$, the $\mathrm{D}_{\mathrm{w}, \mathrm{m}}$ scoring method gives a relatively uniform dose ratio distribution in the PTV in comparison to the $\mathrm{D}_{\mathrm{m}, \mathrm{m}}$ (figure $8 b$ ). Higher density/Z regions at the ribs, where dose attenuation is larger than the surrounding tissues and water, creates a shadowing effect (figure 8a and 8c) where most of the dose is absorbed by the ribs but, as the dose for both methods is scored in water, a lower dose ratio is present for the heterogeneous model inside the ribs. Low density/Z regions, such as adipose tissue, have the reverse effect resulting in an increased dose ratio to regions further from the PTV. As with the Water-Air model in the previous section, there is also a missing backscatter component which reduces the skin and PTV dose. The average $\mathrm{D}_{90}$ to both the Water-Air and heterogeneous models decrease from the $\mathrm{D}_{90}$ calculated using TG-43 MC (Table 3) by about $3.8 \%$ and $5 \%$ respectively. This would seem to indicate that most of the dose reduction is due to the loss of backscatter while only $1.2 \%$ can be attributed to tissue heterogeneities which can be highlighted by the difference between the dose ratio maps in figure $8 \mathrm{a}$ and $8 \mathrm{c}$. Similar reductions can be seen for the other dose metrics (Table 3). Figure $8 \mathrm{~b}$ shows dose ratio maps when $\mathrm{D}_{\mathrm{m}, \mathrm{m}}$ is used for dose reporting. Figure $8 \mathrm{a}$ and $8 \mathrm{~b}$ demonstrate a large difference in the dose ratio distribution due to the difference in scoring method. The figure $8 \mathrm{~b}$ ratio map demonstrates the dependence of $\mathrm{D}_{\mathrm{m}, \mathrm{m}}$ on the tissue type with the dose ratio distribution closely resembling the tree-like pattern of glandular tissue in the egsphant tissue model in figure $8 \mathrm{e}$. The relative difference of the $\mathrm{D}_{\mathrm{m}, \mathrm{m}}$ dose distribution to TG-43 MC is dependent on the tissue composition with glandular tissue and skin absorbing a higher $\mathrm{D}_{\mathrm{m}, \mathrm{m}}$ dose than adipose tissue. By comparison to the $\mathrm{D}_{\mathrm{w}, \mathrm{m}}$ result where tissue heterogeneity only reduced the $\mathrm{D}_{90}$ by $1.2 \%$, if scored with $\mathrm{D}_{\mathrm{m}, \mathrm{m}}$, the tissue heterogeneity reduces the $\mathrm{D}_{90}$ by, on average, $34.8 \%$, a factor 9 more than the contribution of non-patient backscatter (3.8\%). A comparison with figure $8 \mathrm{~d}$ demonstrates the reduced impact of backscatter when scored with $\mathrm{D}_{\mathrm{m}, \mathrm{m}}$. Only dose to rib and the lung region are higher in $\mathrm{D}_{\mathrm{m}, \mathrm{m}}$. The ribs show a dose increase in all patients by approximately $300 \%$ compared to TG-43. An increase in rib dose absorption may not be significant when correlated against osseous toxicity. A previous study(Mandell et al., 1985) had shown no correlation between the increased rib dose absorption of ${ }^{125} \mathrm{I}$ seeds implanted close to cortical bone throughout the body and incidence of bone fractures. ${ }^{125} \mathrm{I}$ is a low energy source with a mean photon energy similar to that of the S700 source. 
(a)Dose ratio: Heterogenous Model $\mathrm{D}_{\mathrm{w}, \mathrm{m}} / \mathrm{TG}-43 \mathrm{MC}$

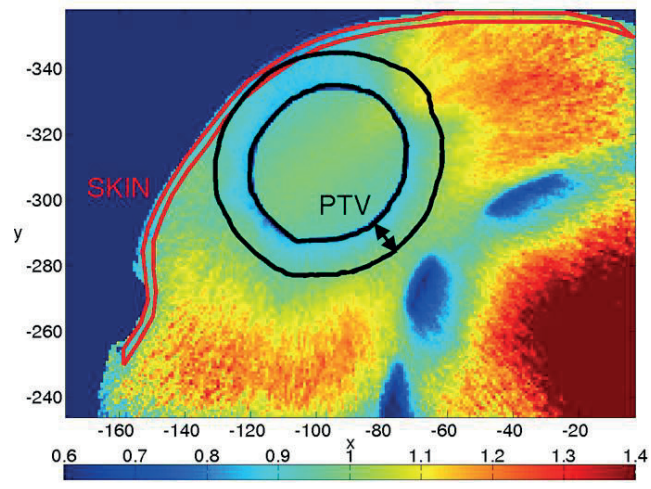

(c) Dose ratio: Heterogenous Model $D_{w, m} /$ Water-Air

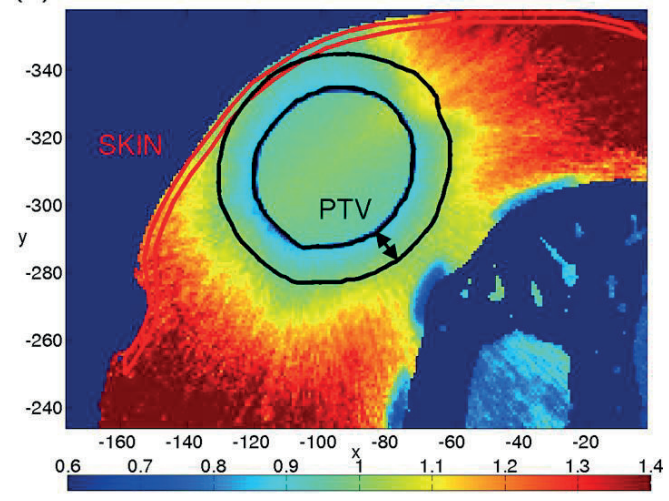

(e)

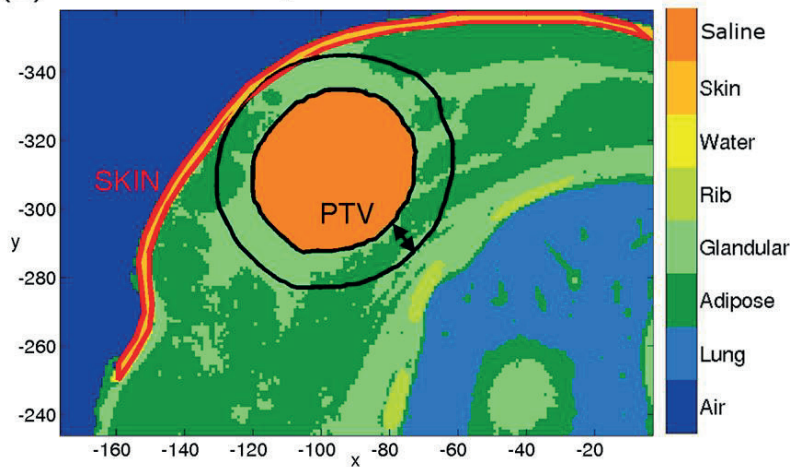

(b) Dose ratio: Heterogenous Model D $\mathrm{mm}$ / TG-43 MC

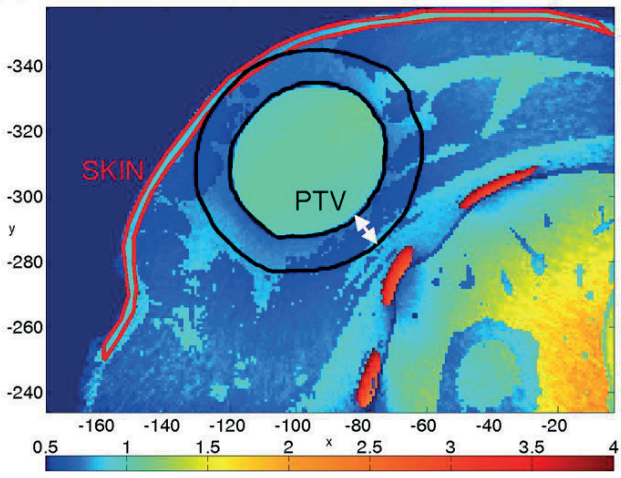

(d)Dose ratio: Heterogenous Model $D_{m m} /$ Water-Air

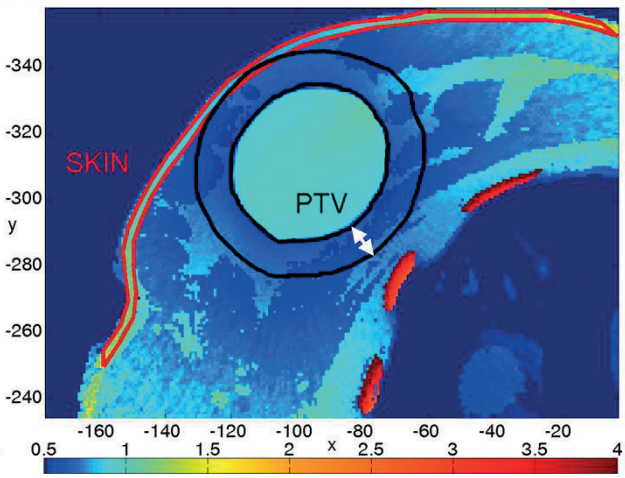

Figure 8: $\mathrm{XY}$ plane dose ratio maps for the heterogeneous model / TG-43 MC scored with $\mathrm{D}_{\mathrm{w}, \mathrm{m}}$ (a) and $\mathrm{D}_{\mathrm{m}, \mathrm{m}}$ (b) and the heterogeneous model / Water-Air Model scored with $\mathrm{D}_{\mathrm{w}, \mathrm{m}}(\mathrm{c})$ and $\mathrm{D}_{\mathrm{m}, \mathrm{m}}$ (d). The contours in all figures are indicative of the ROIs. The dose to the balloon wall is ignored. (e) Slice from the heterogeneous model used for (a)(d).

Note: lung/air dose is ignored in the Water-Air model and all ratios in air are overridden to zero. 
Table 3: Dose metrics for each model / scoring method averaged for 7 patients.

$\mathrm{D}_{90}$ and $\mathrm{D}_{0.2 \mathrm{cc}}$ are expressed as a percentage of the prescription dose, $34 \mathrm{~Gy} . \mathrm{V}_{100}, \mathrm{~V}_{150}$, and $\mathrm{V}_{200}$ are expressed as a percentage of the PTV volume.

\begin{tabular}{l|llll}
\cline { 2 - 5 } & $\begin{array}{l}\text { TG-43-MC model } \\
\left(\mathrm{D}_{\mathrm{w}, \mathrm{w}}\right) \\
\%-\sigma\end{array}$ & $\begin{array}{l}\text { Water + Air model } \\
\left(\mathrm{D}_{\mathrm{w}, \mathrm{w}}\right) \\
\%-\sigma\end{array}$ & $\begin{array}{l}\text { Heterogeneous } \\
\text { model }\left(\mathrm{D}_{\mathrm{w}, \mathrm{m}}\right) \\
\%-\sigma\end{array}$ & $\begin{array}{l}\text { Heterogeneous } \\
\text { model }\left(\mathrm{D}_{\mathrm{m}, \mathrm{m}}\right) \\
\%-\sigma\end{array}$ \\
\hline PTV metric $_{90}$ & $103.9 \pm 2.5$ & $100.1 \pm 2.9$ & $98.9 \pm 3.1$ & $65.3 \pm 2.9$ \\
$\mathrm{~V}_{100}$ & $93.0 \pm 2.0$ & $90.0 \pm 2.5$ & $88.8 \pm 3.3$ & $45.9 \pm 6.1$ \\
$\mathrm{~V}_{150}$ & $49.1 \pm 3.6$ & $46.5 \pm 3.9$ & $39.6 \pm 4.7$ & $13.9 \pm 5.3$ \\
$\mathrm{~V}_{200}$ & $23.5 \pm 3.8$ & $22.1 \pm 4.0$ & $12.5 \pm 5.3$ & $3.4 \pm 2.7$ \\
Skin $\mathrm{D}_{0.2 \mathrm{cc}}$ & $92.9 \pm 32.4$ & $83.0 \pm 33.5$ & $81.4 \pm 27.8$ & $76.9 \pm 25.8$ \\
\hline \hline
\end{tabular}

In terms of inter-patient variability, the largest dose reductions occur in patients with smaller breasts $(<800 \mathrm{cc})$ indicating that the difference between the heterogeneous model and TG-43 are sensitive to geometry as well as to tissue composition. It should be stated that a significant portion of the difference (particularly for $\mathrm{D}_{\mathrm{w}, \mathrm{m}}$ ) between the TG-43 MC and the heterogeneous model is due to balloon wall attenuation which is partially accounted for in clinical practice by a recommended $6 \%$ increase in dwell times. This correction was not applied to the patients in this study. A simulation of the TG-43 MC geometry which included the CADmesh-modeled balloon wall for a single patient showed that the addition of the balloon wall reduced the $\mathrm{D}_{90}\left(\mathrm{D}_{\mathrm{w}, \mathrm{w}}\right)$ by approximately $10 \%$. For the heterogeneous model scored with $D_{w, m}$, this decrease is offset by the lower attenuation coefficient of adipose tissue which compensates the dose to regions around the balloon and increases it further from the applicator (seen in the high dose ratio in figure $8 \mathrm{a}$ and figure $8 \mathrm{c}$ ).

\section{Discussion and study limitations}

We observed a greater degree of variability with an increased level of dose calculation model complexity. When compared to TG-43, the inclusion of the balloon applicator and tissue heterogeneities introduce a number of cold and hot spots which are scoring method dependent. The most significant changes occur near the organs at risk; the skin and ribs. Peak skin dose shows a general decrease over the 7 patients between $0.5 \%$ and $24 \%$ (Table 3 ) of the prescription dose depending on the patient geometry, method of scoring and model type. The reduced skin dose may have implications for dose escalation in APBI as skin dose is a planning constraint. As with the PTV, the choice of dose scoring method is important for determining rib dose with an increase or decrease from TG-43 MC observed with $\mathrm{D}_{\mathrm{m}, \mathrm{m}}$ and $\mathrm{D}_{\mathrm{w}, \mathrm{m}}$, respectively. The differences to the PTV dose observed with $\mathrm{D}_{\mathrm{m}, \mathrm{m}}$ are difficult to evaluate clinically due to the different quantity that both dose scoring metrics represent and the limited experience in clinical evaluations performed using $\mathrm{D}_{\mathrm{m}, \mathrm{m}}$ dosimetry. Within the PTV, the distribution of dose to the specific tissues (glandular/adipose) may be relevant to clinicians.

As reported before(Segala et al., 2011), the balloon wall varies in thickness throughout the applicator. Given the relative low resolution of the CT data it is difficult to determine which sections are thicker/thinner. It would be difficult to incorporate these variations into the CADmesh technique for modeling the balloon wall without a higher resolution CT, and in this study a uniform balloon wall thickness was therefore modeled. 
The study made use of population-averaged healthy tissue composition data rather than the patient's actual composition data. Variation in the tissue composition data $( \pm \sigma)$ has already been shown to cause complex behavior in dose distributions irradiated with the Axxent system(Landry et al., 2010b). Further study is needed to explore the suitability of averaged tissue composition with the Axxent system. Of particular interest is the impact of calcifications throughout the breast which has been shown(Sutherland et al., 2011) to affect the photon fluence in ${ }^{103} \mathrm{Pd}$ treated breast geometries. No calcification distribution was incorporated into the patient phantoms in this study.

With regard to tissue segmentation, the degree of density overlap between glandular and adipose tissues created some assignment uncertainty. Mixed adipose and glandular voxels were not accounted for. The accuracy of the tissue segmentation is also dependent on the CT calibration curve and quality of the images. More precise individual breast compositions, identification of small calcifications and improved tissue segmentation may be possible with dual energy CT imaging(Ding et al., 2013).

\section{Conclusions}

We analyzed the dosimetric impact of tissue heterogeneities and the applicator geometry retrospectively on APBI patients treated with the Xoft Axxent eBx source using MC simulation techniques. TG-43 and TG-186 tissue models were simulated and compared, and results for different dose reporting methods were explored. Simulated TG-186 models demonstrated a difference in predicted dose to all volumes of interest. TG-43 overestimates the doses to certain regions in the body, most notably the skin but underestimates in other regions such as the ribs. These changes are dependent on the dose reporting method. Reduced skin dose may permit dose-escalation and better predict cosmetic outcome. A novel technique for MC modeling of complex geometries based on mesh geometries was used for the first time in brachytherapy. More research is needed to determine the full extent of the role of tissue heterogeneities particularly in terms of clinical outcome. The significant differences between $D_{m, m}$ and $D_{w, m}$ raise questions about the choice of dose reporting metrics, PTV delineation and the role of TG-43U1.

\section{Acknowledgements}

This work is supported by grant \#2011-700810 of the Canadian Cancer Society Research Institute (CCSRI). SW was funded by a MAASTRO-Atrium research grant. GL was supported by the Natural Sciences and Engineering Research Council of Canada (NSERC) and by the O'Brien Foundation. BR was supported by a Marie Curie Reintegration Grant (grant agreement no. PIRG05-GA-2009-247878 from FP7-PEOPLE-2009-RG). Xoft Inc is gratefully acknowledged for providing technical details for the simulations. Special thanks to Mark Podesta for his support with HTCondor and to OpenLine for donating computation time. 


\section{References}

R. Nath, L. L. Anderson, G. Luxton, K. A. Weaver, J. F. Williamson and A. S. Meigooni, "Dosimetry of interstitial brachytherapy sources: recommendations of the AAPM Radiation Therapy Committee Task Group No. 43. American Association of Physicists in Medicine," Med Phys 22, 209-234 (1995).

J.-P. Pignol, B. Keller, E. Rakovitch, R. Sankreacha, H. Easton and W. Que, "First report of a permanent breast 103Pd seed implant as adjuvant radiation treatment for early-stage breast cancer," International journal of radiation oncology, biology, physics 64, 176-181 (2006).

A. Dickler, "Xoft Axxent electronic brachytherapy: a new device for delivering brachytherapy to the breast," Nat Clin Pract Oncol 6, 138-142 (2009).

L. Beaulieu, A. Carlsson Tedgren, J. F. Carrier, S. D. Davis, F. Mourtada, M. J. Rivard, R. M. Thomson, F. Verhaegen, T. A. Wareing and J. F. Williamson, "Report of the Task Group 186 on model-based dose calculation methods in brachytherapy beyond the TG-43 formalism: current status and recommendations for clinical implementation," Med Phys 39, 6208-6236 (2012).

M. J. Rivard, B. M. Coursey, L. A. DeWerd, W. F. Hanson, M. S. Huq, G. S. Ibbott, M. G. Mitch, R. Nath and J. F. Williamson, "Update of AAPM Task Group No. 43 Report: A revised AAPM protocol for brachytherapy dose calculations," Medical Physics 31, 633-674 (2004).

G. Landry, B. Reniers, L. Murrer, L. Lutgens, E. B. Gurp, J. P. Pignol, B. Keller, L. Beaulieu and F. Verhaegen, "Sensitivity of low energy brachytherapy Monte Carlo dose calculations to uncertainties in human tissue composition," Med Phys 37, 5188-5198 (2010).

J. G. H. Sutherland, K. M. Furutani, Y. I. Garces and R. M. Thomson, "Model-based dose calculations for I-125 lung brachytherapy," Medical Physics 39, 4365-4377 (2012).

H. Afsharpour, G. Landry, B. Reniers, J. P. Pignol, L. Beaulieu and F. Verhaegen, "Tissue modeling schemes in low energy breast brachytherapy," Phys Med Biol 56, 7045-7060 (2011).

M. J. Rivard, S. D. Davis, L. A. DeWerd, T. W. Rusch and S. Axelrod, "Calculated and measured brachytherapy dosimetry parameters in water for the Xoft Axxent X-Ray Source: an electronic brachytherapy source," Med Phys 33, 4020-4032 (2006).

D. Liu, E. Poon, M. Bazalova, B. Reniers, M. Evans, T. Rusch and F. Verhaegen, "Spectroscopic characterization of a novel electronic brachytherapy system," Phys Med Biol 53, 61-75 (2008).

T. R. Nelson, L. I. Cervino, J. M. Boone and K. K. Lindfors, "Classification of breast computed tomography data," Med Phys 35, 1078-1086 (2008).

M. J. Yaffe, J. M. Boone, N. Packard, O. Alonzo-Proulx, S. Y. Huang, C. L. Peressotti, A. Al-Mayah and K. Brock, "The myth of the 50-50 breast," Med Phys 36, 5437-5443 (2009).

H. Afsharpour, J. P. Pignol, B. Keller, J. F. Carrier, B. Reniers, F. Verhaegen and L. Beaulieu, "Influence of breast composition and interseed attenuation in dose calculations for post-implant assessment of permanent breast 103Pd seed implant," Phys Med Biol 55, 4547-4561 (2010).

C. Furstoss, B. Reniers, M. J. Bertrand, E. Poon, J. F. Carrier, B. M. Keller, J. P. Pignol, L. Beaulieu and F. Verhaegen, "Monte Carlo study of LDR seed dosimetry with an application in a clinical brachytherapy breast implant," Med Phys 36, 1848-1858 (2009).

International Commission on Radiation Units and Measurements, "Photon, Electron, Proton and Neutron Interaction Data for Body Tissues," (1992).

International Commission on Radiological Protection, "Basic anatomical and physiological data for use in radiological protection: reference values," in ICRP Publication 89 (International Commission on Radiological Protection, New York, NY, 2002).

J. G. H. Sutherland, R. M. Thomson and D. W. O. Rogers, "Changes in dose with segmentation of breast tissues in Monte Carlo calculations for low-energy brachytherapy," Medical Physics 38, 4858-4865 (2011).

S. Agostinelli, J. Allison, K. Amako, J. Apostolakis, H. Araujo, P. Arce, M. Asai, D. Axen, S. Banerjee, G. Barrand, F. Behner, L. Bellagamba, J. Boudreau, L. Broglia, A. Brunengo, H. Burkhardt, S. Chauvie, J. Chuma, R. Chytracek, G. Cooperman, G. Cosmo, P. Degtyarenko, A. Dell'Acqua, G. Depaola, D. Dietrich, R. Enami, A. Feliciello, C. Ferguson, H. Fesefeldt, G. Folger, F. Foppiano, A. Forti, S. Garelli, S. Giani, R. Giannitrapani, D. Gibin, J. J. Gamez Cadenas, I. Gonzailez, G. Gracia Abril, G. Greeniaus, W. Greiner, V. Grichine, A. Grossheim, S. Guatelli, P. Gumplinger, R. Hamatsu, K. Hashimoto, H. Hasui, A. Heikkinen, A. Howard, V. Ivanchenko, A. Johnson, F. W. Jones, J. 
Kallenbach, N. Kanaya, M. Kawabata, Y. Kawabata, M. Kawaguti, S. Kelner, P. Kent, A. Kimura, T. Kodama, R. Kokoulin, M. Kossov, H. Kurashige, E. Lamanna, T. Lampan, V. Lara, V. Lefebure, F. Lei, M. Liendl, W. Lockman, F. Longo, S. Magni, M. Maire, E. Medernach, K. Minamimoto, P. Mora de Freitas, Y. Morita, K. Murakami, M. Nagamatu, R. Nartallo, P. Nieminen, T. Nishimura, K. Ohtsubo, M. Okamura, S. O'Neale, Y. Oohata, K. Paech, J. Perl, A. Pfeiffer, M. G. Pia, F. Ranjard, A. Rybin, S. Sadilov, E. Di Salvo, G. Santin, T. Sasaki, N. Savvas, Y. Sawada, S. Scherer, S. Sei, V. Sirotenko, D. Smith, N. Starkov, H. Stoecker, J. Sulkimo, M. Takahata, S. Tanaka, E. Tcherniaev, E. Safai Tehrani, M. Tropeano, P. Truscott, H. Uno, L. Urban, P. Urban, M. Verderi, A. Walkden, W. Wander, H. Weber, J. P. Wellisch, T. Wenaus, D. C. Williams, D. Wright, T. Yamada, H. Yoshida and D. Zschiesche, "Geant4: a simulation toolkit," Nuclear Instruments and Methods in Physics Research Section A: Accelerators, Spectrometers, Detectors and Associated Equipment 506, 250-303 (2003).

H. J. Cullen D, Kissel L., "EPDL97: The Evaluated Photon Data Library, 97 version,," LLNL Report No. UCRL-50400 Vol. 6 (1997).

S. C. Perkins, DE. Chen, MH. Hubbell, JH. Rathkopf, JH. Scofield, J., "Table and graphs of atomic subshell and relaxation data derived from the LLNL Evaluated Atomic Data Library (EADL)," LLNL Report No. UCRL-50400 Vol. 30 (1997).

J. F. Williamson, "Monte Carlo evaluation of kerma at a point for photon transport problems," Med Phys 14, 567-576 (1987).

T. T. Douglas, Tannenbaum. Miron, Livny., "Distributed computing in practice: the Condor experience.," Concurrency - Practice and Experience 17, 323-356 (2005).

E. Poon, Y. Le, J. F. Williamson and F. Verhaegen, "BrachyGUI: an adjunct to an accelerated Monte Carlo photon transport code for patient-specific brachytherapy dose calculations and analysis," Journal of Physics: Conference Series 102, 012018 (2008).

I. R. Kawrakow, D.W.O. Walters, B.R.B., DOSXYZnrc Users Manual. (NRCC,Ottawa, ON, Canada, 2007).

S. D. Davis, PhD, University of Wisconsin, 2009.

Xoft, "Axxent Controller Operator Manual-Model 110," (2009).

J. A. Raffi, T. L. Pike, S. D. Davis and L. A. DeWerd, "Comparison of Measured and Calculated Exit Dose for Intracavitary Accelerated Partial Breast Irradiation with an Electronic Brachytherapy Source," Brachytherapy 9, Supplement 1, S36 (2010).

J. J. Segala, G. A. Cardarelli, J. R. Hiatt, B. H. Curran and E. S. Sternick, "Interface dosimetry for electronic brachytherapy intracavitary breast balloon applicators," J Appl Clin Med Phys 12, 3221 (2011).

S. A. Enger, G. Landry, M. D'Amours, F. Verhaegen, L. Beaulieu, M. Asai and J. Perl, "Layered mass geometry: a novel technique to overlay seeds and applicators onto patient geometry in Geant4 brachytherapy simulations," Phys Med Biol 57, 6269-6277 (2012).

J. A. Apostolakis, M. ; Cosmo, G. ; Howard, A. ; Ivanchenko, V. ; Verderi, M., "Parallel geometries in Geant4: foundation and recent enhancements," IEEE Nucl. Sci. Symp. Conf. Record (NSS '08), pp 883-886 (2008).

C. M. Poole, I. Cornelius, J. V. Trapp and C. M. Langton, "A CAD interface for GEANT4," Australas Phys Eng Sci Med 35, 329-334 (2012).

S. J. v. Hoof, P. V. Granton, G. Landry, M. Podesta and F. Verhaegen, "Evaluation of a novel triple-channel radiochromic film analysis procedure using EBT2," Physics in Medicine and Biology 57, 4353 (2012).

A. Budrukkar, V. Jagtap, S. Kembhavi, A. Munshi, R. Jalali, T. Seth, V. Parmar, R. Raj Upreti, R. Badwe and R. Sarin, "Fat necrosis in women with early-stage breast cancer treated with accelerated partial breast irradiation (APBI) using interstitial brachytherapy," Radiotherapy and Oncology 103, 161-165 (2012).

D. E. Wazer, D. Lowther, T. Boyle, K. Ulin, A. Neuschatz, R. Ruthazer and T. A. DiPetrillo, "Clinically evident fat necrosis in women treated with high-dose-rate brachytherapy alone for early-stage breast cancer," International journal of radiation oncology, biology, physics 50, 107-111 (2001).

J. A. Vargo, V. Verma, H. Kim, R. Kalash, D. E. Heron, R. Johnson and S. Beriwal, "Extended (5-year) Outcomes of Accelerated Partial Breast Irradiation Using MammoSite Balloon Brachytherapy: Patterns of Failure, Patient Selection, and Dosimetric Correlates for Late Toxicity," International Journal of Radiation Oncology*Biology*Physics 88, 285-291 (2014).

J. Perez-Calatayud, D. Granero and F. Ballester, "Phantom size in brachytherapy source dosimetric studies," Medical Physics 31, 2075-2081 (2004). 
L. Mandell, D. Nori, L. Anderson, M. Belanich and B. Hilaris, "The effects of permanent I-125 interstitial implantation on cortical bone," International Journal of Radiation Oncology Biology Physics 11, 10291034 (1985).

H. Ding, J. L. Ducote and S. Molloi, "Measurement of breast tissue composition with dual energy cone-beam computed tomography: A postmortem study," Medical Physics 40, 061902 (2013). 



\section{Chapter 4:}

\section{Measurement of absorbed dose to water around an}

electronic brachytherapy source. Comparison of two dosimetry systems: lithium formate EPR dosimeters and radiochromic EBT2 film

Emelie Adolfsson, Shane White, Guillaume Landry, Eva Lund, Håkan Gustafsson, Frank

Verhaegen, Brigitte Reniers, Åsa Carlsson Tedgren' Gudrun Alm Carlsson 


\begin{abstract}
Purpose

Interest in HDR electronic brachytherapy operating at $50 \mathrm{kV}$ is increasing. For quality assurance it is important to identify dosimetry systems that can measure the absorbed doses in absolute terms which is difficult in this energy region. In this work a comparison is made between two dosimetry systems, EPR lithium formate dosimeters and radiochromic EBT2 film.
\end{abstract}

\title{
Methods and Materials
}

Both types of dosimeters were irradiated simultaneously in a PMMA phantom using the Axxent EBS. Absorbed dose to water was determined at distances of $10 \mathrm{~mm}, 30 \mathrm{~mm}$ and 50 $\mathrm{mm}$ from the EBS. Results were traceable to different primary standards as regards absorbed dose to water (EPR) and air kerma (EBT2). Monte Carlo simulations were used in absolute terms as a third estimate of absorbed dose to water.

\section{Results:}

Agreement within the estimated expanded $(\mathrm{k}=2)$ uncertainties (5\% (EPR), 7\% (EBT2)) was found between the results at $30 \mathrm{~mm}$ and $50 \mathrm{~mm}$ from the x-ray source. The same result was obtained in 4 repetitions of irradiation, indicating high precision in the measurements with both systems. At $10 \mathrm{~mm}$ distance, agreement between EPR and Monte Carlo simulations was shown, while the geometry for the film measurement caused too large uncertainty in measured values which depend on the exact position (within sub-mm distances) of the EBS.

\section{Conclusions:}

This work has demonstrated good performance of the lithium formate EPR dosimetry system in accordance with earlier experiments at higher photon energies $\left({ }^{192} \operatorname{Ir}\right.$ HDR brachytherapy). Results for the EBT2 film agreed with the results of Monte Carlo simulations of absorbed dose to water at $30 \mathrm{~mm}$ and $50 \mathrm{~mm}$ distance. It was also highlighted that there might be issues regarding the energy dependence and intrinsic efficiency of the EBT2 film that need to be considered for measurements using low energy sources. 


\section{Introduction}

Interest in using high dose rate (HDR) electronic x-ray brachytherapy sources (EBS) for accelerated partial breast irradiation (APBI) or intraoperative radiation therapy (IORT) is increasing. Due to the low tube voltage $(40-50 \mathrm{kV})$ the need for radiation shielding of treatment rooms is reduced. There are also studies showing how the use of EBS for APBI results in reduced doses to normal tissues compared to ${ }^{192}$ Ir HDR. However, the issue is complicated by the sensitivity of calculated doses to patient tissue composition in this energy region (Mille et al., 2010; White et al., 2014a).

Experimental verification of dose distributions is an important feature of quality assurance in radiation therapy. It is difficult to perform dosimetric measurements around low energy $(<50$ $\mathrm{keV}$ ) brachytherapy sources. Not only are dose gradients very steep, making accurate positioning and control over dose-averaging effects important, but the attenuation of photons in the keV energy region is also very sensitive to phantom material composition. It is furthermore not clear if calibrations to measure absorbed dose to water in absolute terms should be made using traceability to a primary water standard using a MV photon beam or to an air kerma standard in a $\mathrm{kV}$ radiation quality closer to that of the EBS. The highest accuracy in calibration coefficients in MV beams is achieved using traceability to a primary ${ }^{60}$ Co water standard (Almond et al., 1999; Andreo et al., 2000). Calibrating absorbed dose in MV beams and measuring in $\mathrm{kV}$ beams, however, generally requires correcting the dosimetry system for effects of intrinsic efficiency. Intrinsic efficiency is defined as the signal per mean absorbed dose in the detector and should not be confused with the term energy dependence defined as the signal per absorbed dose to water. Various LiF thermoluminescent dosimeter (TLD) systems have shown a response per unit absorbed dose in the detector that is about 5\%-10\% higher in 20-50 kV beams than in MV beams (Carlsson Tedgren et al., 2011; Davis et al., 2003; Nunn et al., 2008). On the contrary, the lithium formate electron paramagnetic resonance (EPR) dosimetry system used in this work shows an approximately $5 \%$ lower response in $\mathrm{kV}$ than in MV beams (Adolfsson et al., 2010). The effect of variations of intrinsic efficiency with beam quality is often neglected and has not been investigated for many dosimetry systems including EBT2 radiochromic film in photon beams. This precludes calibration in a MV beam to assure accurate dosimetry.

Our lithium formate EPR dosimetry system has been used successfully for measurements of absorbed dose to water around ${ }^{192}$ Ir HDR BT sources (Antonovic et al., 2009). The aim of this work was to test this dosimetry system for measurements of absorbed dose to water around a low energy $50 \mathrm{kV}$ Axxent EBS (Xoft Inc, San Jose, CA, USA) (Rivard et al., 2006a) and to compare the results against an independent dosimetry system using radiochromic EBT2 film. 


\section{Materials and methods}

This section is structured as follows: The theory underlying the derivation of absorbed dose to water from the measured dosimeter signal at various depths in a phantom is treated in 2.1. Measurement techniques and calibration methods to determine absorbed dose to water are described for the EPR dosimeters in 2.2 and for the EBT2 film in 2.3; the geometrical set-up for the measurements with the EBS is described in 2.4. Monte Carlo simulations to derive the energy-spectra of photons at different depths in the measurement phantom, a method to convert the results of MC calculations into absolute dose values including necessary correction factors are described in 2.5.

\section{Theory}

\section{Derivation of absorbed dose to water from the measured signal}

Determination of absorbed dose to water at beam quality Q, at the distance $r$ from the EBS, is given by

$$
\begin{aligned}
D_{\mathrm{w}, \mathrm{Q}}=D_{\mathrm{w}}(r) & =\frac{M_{\mathrm{Q}}}{\left[\frac{M}{D_{\mathrm{w}}}\right]_{\mathrm{Q}_{0}}} \frac{\left[\frac{M}{D_{\mathrm{w}}}\right]_{\mathrm{Q}_{0}}}{\left[\frac{M}{D_{\mathrm{w}}}\right]_{\mathrm{Q}}}=\frac{M_{\mathrm{Q}}}{\left[\frac{M}{D_{\mathrm{w}}}\right]_{\mathrm{Q}_{0}}} \frac{\left[\frac{M}{\bar{D}_{\text {det }}}\right]_{\mathrm{Q}_{0}}}{\left[\frac{M}{\bar{D}_{\text {det }}}\right]_{\mathrm{Q}}} \frac{\left[\frac{\bar{D}_{\text {det }}}{D_{\mathrm{w}}}\right]_{\mathrm{Q}_{0}}}{\left[\frac{\bar{D}_{\text {det }}}{\bar{D}_{\mathrm{w}}}\right]_{\mathrm{Q}}}= \\
& =\frac{M_{\mathrm{Q}}}{\left[\frac{M}{D_{\mathrm{w}}}\right]_{\mathrm{Q}_{0}}} \frac{R_{\mathrm{Q}_{0}}}{R_{\mathrm{Q}}} \frac{\left[\frac{\bar{D}_{\text {det }}}{D_{\mathrm{w}}}\right]_{\mathrm{Q}_{0}}}{\left[\frac{\bar{D}_{\text {det }}}{D_{\mathrm{w}}}\right]_{\mathrm{Q}}}=\frac{M}{\left[\frac{M}{D_{\mathrm{w}}}\right]_{\mathrm{Q}_{0}}} k_{\mathrm{Q}_{\mathrm{Q}}}
\end{aligned}
$$

Here $M_{\mathrm{Q}}$ is the detector signal at beam quality $\mathrm{Q},\left[M / D_{\mathrm{w}}\right]_{\mathrm{Q}_{0}}$ is the signal per unit absorbed dose to water at the calibration beam quality $\mathrm{Q}_{0}, R=M / \bar{D}_{\text {det }}$ is the signal per mean absorbed dose in the detector and is generally a function of the beam quality as indicated by the indices $\mathrm{Q}$ and $\mathrm{Q}_{0}$. The quotient $R_{Q_{0}} / R_{Q}$ corrects for different intrinsic efficiency of the detector material at calibration and at measurement. $k_{Q, Q_{0}}$ is the beam quality correction factor.

Corrections were needed to convert the mean absorbed dose in the detector, $\bar{D}_{\text {det }}$ (proportional to the measured signal) to the absorbed dose, $D_{\operatorname{det}}(r)$ in a small detector at points $r$, corresponding to the distances from the EBS at which the absorbed dose to water was measured. Details of the expressions used for determining the absorbed dose to water with the two detector systems and uncertainty estimations are given in the Appendix.

\section{Relative intrinsic efficiency $R_{Q_{0}} / R_{Q}$}

The intrinsic efficiency depends on the ionization densities and varies due to the linear energy transfer (LET) distribution of the secondary electrons. Intrinsic efficiency at brachytherapy beam qualities relative to ${ }^{60} \mathrm{Co}$ or $\mathrm{MV}$ photon beam has been thoroughly investigated for 
lithium formate EPR dosimeters (Adolfsson et al., 2010). No corresponding investigations have to our knowledge been performed for radiochromic film. A complicating factor for the film is its nonlinear dose response, i.e. $M / D_{\mathrm{w}}$ and thus $M / \bar{D}_{\text {det }}$ varies not only with the LET distributions along single electron tracks but also with the magnitude of the absorbed dose in the detector with ionization densities in sensitive targets formed by overlapping electron tracks.

\section{Energy absorption properties relative to water}

From knowledge of $M / D_{\mathrm{w}}, M / \bar{D}_{\text {det }}$ could be determined provided $D_{\mathrm{w}} / \bar{D}_{\text {det }}$ is known. Values of the latter quotient have until recently been unknown for radiochromic film due to lack of knowledge of the concentrations of high atomic number materials used to increase film sensitivity (Lindsay et al., 2010). Sutherland and Rogers (Sutherland and Rogers, 2010) provided calculations of $D_{\mathrm{w}} / \bar{D}_{\text {det }}$ using photon-electron transport Monte Carlo simulations and found the ratio to be close to the ratio of the mass energy absorption coefficients of the active film layer and water at photon energies $<50 \mathrm{keV}$. This indicates a state of charged particle equilibrium in the active layer at these energies. It is noted that at higher photon energies this does not hold and full photon-electron transport must be simulated to obtain the mean absorbed dose in the detector.

Since May 2009, the manufacturer of EBT2 film (ISP) has standardized the atomic composition of the active layer (Lindsay et al., 2010). Film lot \# A090310 was used in our experiment, atomic composition of the active layer is given in Table 1 (as provided by ISP, personal communication). Comparison of the ratios between the mass energy absorption coefficients for water and the active layer of three different batches of EBT2 film plus lithium formate are shown in Figure 1. Mass energy absorption coefficients were taken from NIST (Hubbell and Seltzer, 1995).

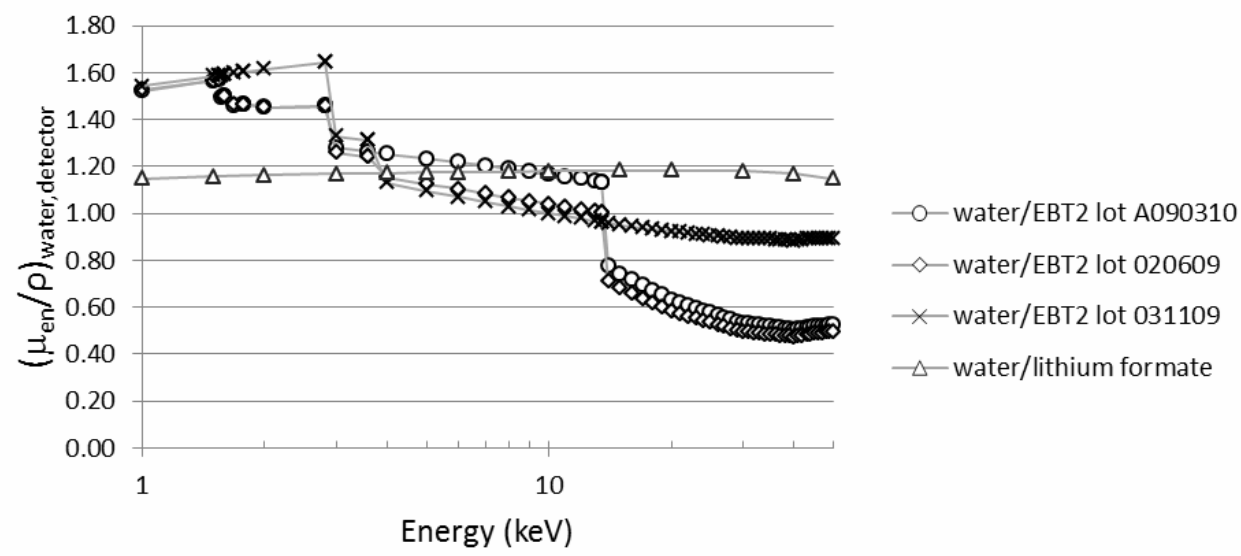

Figure 1. Mass energy absorption coefficients of water relative to lithium formate and the active layers of 3 different lots of EBT2 film; A090310 (used in this work) and 020609, 031109 investigated by Sutherland and Rogers (2010). Atomic compositions by Sutherland and Rogers were obtained from the manufacturer. 
Figure 1 shows that the energy absorption of lithium formate relative to water vary considerably less with photon energy than the variations among the three EBT2 lots. Values of $\left[\bar{\mu}_{\mathrm{en}} / \rho\right]_{\text {det }}^{\mathrm{w}}$ (weighted over the energy fluences calculated for the Axxent source, see 2.5) for lithium formate were within $0.5 \%$ at the three measurement positions, while the corresponding values for lot A090310 were within 4\%.

Results for A090310 used in this work coincide with lot 020609 as simulated by Sutherland and Rogers. It is noted that the standardized composition of the active layer results in a dosimeter with a larger energy dependence $\left(Z_{\text {eff }}=9.18\right)$ than lot $031109\left(Z_{\text {eff }}=7.44\right)$ at these low photon energies (Sutherland and Rogers, 2010).

\section{Calibration methods}

Calibration to measure absorbed dose to water with traceability to an absorbed dose to water primary standard presently requires the use of ${ }^{60} \mathrm{Co}$ or a MV photon beams. An alternative method with traceability to an air kerma standard was used for the film to avoid effects of the unknown intrinsic efficiency on the results of the dose measurements. Details of the calibrations of the EPR dosimeters and of the EBT2 film are given below.

\section{Lithium formate EPR dosimeters}

\section{Measurement technique}

The lithium formate dosimeters were cylindrical in shape with height $4.8 \mathrm{~mm}$ and diameter $4.5 \mathrm{~mm}$. They consisted of $90 \%$ lithium formate and 10\% paraffin (binding material). Signal evaluation was performed with a Bruker EleXsys E580 spectrometer, for details see earlier publications (Adolfsson et al., 2010; Adolfsson et al., 2012; Antonovic et al., 2009; Gustafsson et al., 2008).

\section{Calibration process}

Absorbed dose to water calibration was performed in a $6 \mathrm{MV}$ photon beam using a Farmer type ion chamber. Due to the linear dose response of lithium formate, a two point calibration curve was used (Gustafsson et al., 2008). The corrections necessary to convert absorbed dose to water in the calibration situation to absorbed dose to water at a specified distance from the EBS are described in the Appendix.

Two sets each of four dosimeters each were used for the calibration. One set was calibrated (in Linköping) before the EBS experiment (in Maastricht) and one set afterwards due to the time between experiment and read out of the EPR dosimeters. The results of the calibrations were the same and indicate the stability of the EPR dosimeters throughout the experiment and calibrations (Adolfsson et al., 2014; Adolfsson et al., 2012).

\section{Radiochromic film}

\section{Measurement technique}

The film was evaluated using a flatbed scanner Epson PRO V740 (Seiko Epson Corporation, Nagano, Japan). Image Acquisition was performed using FilmQA Pro (Version 1.0.4049.36336). All film pieces were scanned and the results were stored as TIFF images, 
without any colour correction, at 225dpi in words of 48 bits (16 bits per colour channel) using the RGB transmission mode for the positive film. Absorbed dose to water was derived using the triple channel method (Micke et al., 2011; van Hoof et al., 2012). The film was kept in light-tight folders until use and while in use, exposure to light was kept to a minimum.

\section{Calibration process}

The absorbed dose to water film calibration was performed using a small animal microirradiator with tube voltage $50 \mathrm{kV}, 2.14 \mathrm{~mm} \mathrm{Al}$ filtration and $\mathrm{HVL}=1.54 \mathrm{~mm} \mathrm{Al}$ (first layer). (PXi, North Branford, CT) (Granton et al., 2012; van Hoof et al., 2012). The Farmer chamber was used with traceability to a primary air kerma standard. To determine absorbed dose to water, the "in-air method" described by the AAPM task group 61 (Ma et al., 2001) was used with slight modifications. The micro-irradiator was to too small to use the recommended $10 \times 10 \mathrm{~cm}$ field and the recommend $100 \mathrm{~cm}$ source to detector distance. Instead the field size was limited to a circular $2.5 \mathrm{~cm}$ diameter field and the source to detector distance was $30.7 \mathrm{~cm}$ (the distance to the isocentre) as described in Hoof et al., 2012The calibration curve consisted of 8 dose points from 0 to $15 \mathrm{~Gy}$. A full description of the micro-irradiator and the film calibration process is available elsewhere (van Hoof et al., 2012). The corrections necessary to convert absorbed dose to water in the calibration situation to absorbed dose to water at a specified distance from the EBS are described in the appendix.

\section{Experimental set up for measurement in the EBS beam}

A PMMA phantom previously used for EPR measurements around ${ }^{192} \mathrm{Ir}$ sources (Antonovic et al., 2009) was used for the measurements, see Figure 2. The phantom is divided into square slabs of thickness $2.5 \mathrm{~cm}$ stacked on one another with a centrally drilled hole $(5.1 \mathrm{~mm}$ in diameter). The EBS fitted tightly into the hole with some compression on the sides. It was inserted to the maximum depth of the hole at each time; the positioning (as indicated by markers on the source tip) was accurate to within $<1 \mathrm{~mm}$. The center of the source and the center of the EPR chips were slightly out of plane by approximately $1 \mathrm{~mm}$. This was revealed in noting updated technical information about the source received after the experiment. (The updated information was taken into account when calculating the correction factors (see 2.5)). At each distance $(10,30$ and $50 \mathrm{~mm})$ from the EBS, four EPR dosimeters were positioned radially around the EBS (Figure $2 b$ ). Film pieces were positioned on top of the EPR dosimeters, a circular area on the part of the film that covered the underlying EPR dosimeter cross section was marked as the region of interest. The experiment was repeated four times at 30 and $50 \mathrm{~mm}$ (average irradiation time was $550 \mathrm{~s}$ for the four repetitions). One irradiation was made at $10 \mathrm{~mm}$ (irradiation time $88.5 \mathrm{~s}$ ). Due to their limited lifespan, the irradiations were performed using two EBSs. A Markus M6 ion chamber (PTW, Freiburg, Germany) situated below the dosimeter slab was used to monitor the dose delivery. The dosimeters at 30 and $50 \mathrm{~mm}$ were simultaneously irradiated; irradiations of dosimeters at $10 \mathrm{~mm}$ were performed separately to avoid dose saturation of the film. The ramp-up dose of the EBS was determined to be less than $0.2 \%$ of the total administrated dose. 


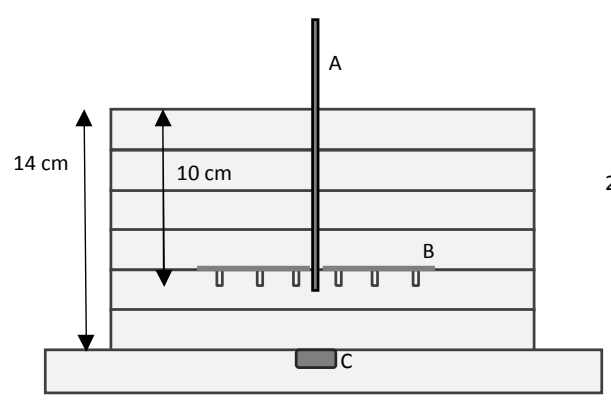

a)

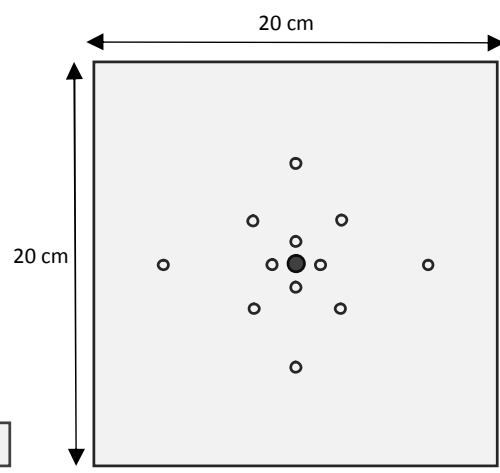

b)

Figure 2. Schematic figure of the PMMA phantom seen from the side (a) and from above (b). Dosimeters were positioned at 10, 30 and $50 \mathrm{~mm}$ from the EBS (A) and the film (B) on top of the EPR dosimeters between two PMMA slabs. The Markus chamber (C) was positioned below the film dosimeter plane. Not drawn to scale. Four dosimeters were simultaneously irradiated at each distance.

\section{Monte Carlo simulations}

Monte Carlo simulations were used in the derivation of corrections factors (see Appendix), to enable comparison of doses determined by EPR and film, and for calculations of the radial absorbed dose profile in the phantom. The latter dose distribution was translated into values of absorbed dose in terms of Gy using the Markus chamber (see 2.5.3).

\section{The Geant4 MC program}

The all-purpose MC simulation toolkit Geant4 v9.3 provides a diverse set of software components that can be employed in a variety of settings, including the simulation of photon transport from low energy sources (Agostinelli et al., 2003c). In this work, the Lawrence Livermore National Laboratory low energy electromagnetic model which simulates the low energy photon interactions of the photoelectric effect, Compton scattering and Rayleigh scattering was used. The electron atomic binding effects on Compton cross sections at low energies were taken into account. The model uses the EPDL (Cullen et al., 1997), and EADL (Perkins et al., 1997) evaluated cross section libraries. All secondary electrons were made to deposit their energy locally due to their short range compared to typical voxel sizes; hence absorbed dose to water is approximated by water collision kerma. The cutoff energy for photons was $250 \mathrm{eV}$.

\section{The geometry}

A mathematical model of the PMMA phantom, EPR dosimeters and films used in the experiment was built with Geant4. The atomic compositions of the materials used are shown in table 1. The EBS source (Axxent S700) was carefully modelled in Geant4 (Agostinelli et al., 2003) and validated by comparing measured and simulated TG-43 parameters in Rivard et al. (2006). Using this model, $50 \mathrm{keV}$ electrons were set in motion at the cathode and transported to a tungsten anode. Photons were generated via bremsstrahlung splitting (number of secondary photons equal to 100) and characteristic x-ray generation. Photons that reach the outer cooling water sheath of the source (diameter $5.1 \mathrm{~mm}$ ) were recorded into a phase space 
file and then terminated. $10^{9}$ primary electrons were simulated to generate a phase space file with $7 \times 10^{7}$ photons. No electrons were recorded due to their short range. The phase space source was validated against TG-43 data from Rivard et al, (2006) to within tolerated uncertainties $(<3 \%)$. This phase space source was used to simulate photon emission from the $\mathrm{S} 700$ in this work and has been used previously (White et al., 2014b).

Three separate simulations for each distance were run utilizing track-length scoring and two billion photon histories for each simulation. The values of photon energy fluence differential with respect to energy obtained in the simulations were used with mass-energy absorption coefficients for water to derive values of absorbed dose to water under conditions of charged particle equilibrium.

Table 1. Atomic composition of materials used in the MC simulations.

\begin{tabular}{llllllllll}
\hline \multirow{2}{*}{ Material } & \multicolumn{9}{l}{ Atomic composition (percentage by weight) } \\
& $\mathbf{H}$ & $\mathbf{C}$ & $\mathbf{O}$ & $\mathbf{L i}$ & $\mathbf{N}$ & $\mathbf{A r}$ & $\mathbf{B r}$ & $\mathbf{N a}$ & $\mathbf{C l}$ \\
\hline Lithium formate & 4.32 & 17.16 & 68.59 & 9.92 & - & - & - & - & - \\
Paraffin & 14.98 & 85.02 & - & - & - & - & - & - & - \\
EBT2 sensitive layer & 9.55 & 58.2 & 28.3 & 0.92 & 0.23 & - & 1.31 & 0.38 & 1.15 \\
lot \# A090310 & - & 0.012 & 23.18 & - & 75.53 & 1.28 & - & - & - \\
Air & 8.05 & 59.98 & 31.97 & - & - & - & - & - & - \\
PMMA & & & & & & & & & \\
\hline
\end{tabular}

\section{Converting Monte Carlo results to absolute dose values}

Monte Carlo simulations were used for a third estimate of ${ }^{{ }_{\text {w }}}$ in terms of Gy. Relative MC doses in terms of fGy/particle were coupled to absorbed dose to water (in Gy) at $30 \mathrm{~mm}$ from the EBS, determined by an independent film irradiation (unpublished results). At this irradiation, the film was positioned $30 \mathrm{~mm}$ from the EBS in a separate PMMA phantom with the Markus chamber perpendicularly to the beam axis. MC simulations were used to determine the relationship between the readings of the Markus chamber in the two geometries, allowing transfer of the absorbed dose to water determined in the independent film irradiation to the absorbed dose to water at $30 \mathrm{~mm}$ in the present experiment.

\section{Results}

Table 2 shows absorbed dose to water as determined by the three different systems and Table 3 shows the quotients between the results of each system. In both film and EPR dosimetry systems, the absorbed dose was determined as the average of four detectors distributed around the source to minimize positional uncertainties (see Figure 2). At $10 \mathrm{~mm}$ distance, the geometry for the film measurement caused large uncertainty in the measured values depending on the exact position (within sub-mm distances) of the EBS, therefore, the film measurement at $10 \mathrm{~mm}$ was left out. The EPR measurements at $10 \mathrm{~mm}$ were considerably less influenced by the EBS position, within 3\% for an uncertainty in the EBS position of \pm 1 $\mathrm{mm}$ relative to the symmetry axis of the EPR dosimeters, the corresponding value for the film was $22 \%$. 
Table 2. Results of absorbed dose to water determined by EPR dosimeters, EBT2 film and Monte Carlo simulations. The expanded $(\mathrm{k}=2)$ relative uncertainties were $5 \%(\mathrm{EPR})$ and $7 \%$ (film) except at $10 \mathrm{~mm}$ where the measurement uncertainty was $6 \%$ for EPR. Uncertainty in the MC simulated doses is estimated to $7 \%$. See Table A1 in appendix for details.

\begin{tabular}{|c|c|c|c|c|c|c|c|c|c|}
\hline \multirow[b]{2}{*}{ Repetition } & \multicolumn{3}{|c|}{ EPR } & \multicolumn{3}{|c|}{ Film } & \multicolumn{3}{|c|}{ MC } \\
\hline & $10 \mathrm{~mm}$ & $30 \mathrm{~mm}$ & $50 \mathrm{~mm}$ & $10 \mathrm{~mm}$ & $30 \mathrm{~mm}$ & $50 \mathrm{~mm}$ & $10 \mathrm{~mm}$ & $30 \mathrm{~mm}$ & $50 \mathrm{~mm}$ \\
\hline 1 & 9.4 & 10.7 & 2.6 & - & 10.1 & 2.6 & 9.4 & 10.3 & 2.4 \\
\hline 2 & & 10.5 & 2.5 & & 10.0 & 2.6 & & 10.2 & 2.4 \\
\hline 3 & & 10.8 & 2.6 & & 10.3 & 2.7 & & 10.5 & 2.5 \\
\hline 4 & & 9.6 & 2.3 & & 9.1 & 2.4 & & 9.3 & 2.2 \\
\hline
\end{tabular}

Table 3. The quotient of results displayed in Table 2.

\begin{tabular}{|c|c|c|c|c|c|c|c|c|c|}
\hline \multirow[b]{2}{*}{ Repetition } & \multicolumn{2}{|c|}{$\mathbf{D}_{w}(\mathbf{E P R}) / \mathbf{D}_{w}(\mathbf{M C})$} & \multicolumn{4}{|c|}{$\mathbf{D}_{w}(\mathrm{film}) / \mathbf{D}_{w}(\mathbf{M C})$} & \multicolumn{3}{|c|}{$\mathbf{D}_{w}(\mathrm{EPR}) / \mathbf{D}_{w}(\mathrm{film})$} \\
\hline & $10 \mathrm{~mm}$ & $30 \mathrm{~mm}$ & $50 \mathrm{~mm}$ & $10 \mathrm{~mm}$ & $30 \mathrm{~mm}$ & $50 \mathrm{~mm}$ & $10 \mathrm{~mm}$ & $30 \mathrm{~mm}$ & $\begin{array}{l}\mathbf{5 0} \\
\mathbf{m m}\end{array}$ \\
\hline 1 & 1.01 & 1.04 & 1.08 & - & 0.98 & 1.08 & - & 1.05 & 0.99 \\
\hline 2 & & 1.03 & 1.05 & & 0.98 & 1.09 & & 1.05 & 0.96 \\
\hline 3 & & 1.03 & 1.07 & & 0.97 & 1.09 & & 1.05 & 0.98 \\
\hline 4 & & 1.03 & 1.05 & & 0.98 & 1.08 & & 1.05 & 0.97 \\
\hline
\end{tabular}

Agreement within the estimated expanded $(\mathrm{k}=2)$ uncertainties (see Appendix) was found between the results obtained with the two dosimetry systems at $30 \mathrm{~mm}$ and $50 \mathrm{~mm}$. This implies that the use of the two different calibration methodologies worked satisfactorily. The agreement between the results with the two dosimetry systems at repeated irradiations is noticeably good indicating that the type A statistical uncertainties are small for both systems. At $10 \mathrm{~mm}$ distance the EPR agreed with the MC calculated value within the estimated uncertainty.

\section{Discussion}

\section{Monte Carlo calculations of absorbed dose to water}

The inclusion of film measurements from a separate experiment to convert the MC calculations to absorbed dose to water in absolute values (Gy) may have introduced a bias of the $\mathrm{MC}$ results as indicated by the good agreement between the MC calculated and film dose values at $30 \mathrm{~mm}$ (the depth of normalization (2.5.3)). The agreement between the results obtained by the EPR dosimeters and the EBT2 films in this study (Tables 2-3) supports the validity of the adopted approach. Uncertainties in the MC calculated values are dominated by the uncertainty in the value for absorbed dose to water that was determined using film dosimetry. 


\section{Energy dependence and intrinsic efficiency of EBT2 film dosimeters}

Using the results of Sutherland and Rogers 2010, it was possible to calculate the absorbed dose in the active film layer allowing analysis of the energy dependence of $M / D_{\mathrm{w}}$ published using the new standardized film in terms of $M / \bar{D}_{\text {det }}$. Brown et al. (Brown et al., 2012) investigated the energy response of monochromatic $\mathrm{x}$-rays $(25,30$ and $35 \mathrm{keV})$ with a $4 \mathrm{MV}$ clinical photon beam as reference. Their results indicate variations in relative intrinsic efficiencies of $R_{25 \mathrm{keV}} / R_{35 \mathrm{keV}}=0.95$ and $R_{25 \mathrm{keV}} / R_{4 \mathrm{MV}}=0.63$. For a $50 \mathrm{kV}$ x-ray beam $(25.5$ $\mathrm{keV}$ effective energy) relative to a $6 \mathrm{MV}$ photon beam (effective energy $1.4 \mathrm{MeV}$ ) results by Butson et al. (Butson et al., 2010) indicate a relative intrinsic efficiency of $R_{50 \mathrm{kV}} / R_{1.4 \mathrm{MeV}}=0.57$, consistent with the result $R_{25 \mathrm{keV}} / R_{4 \mathrm{MV}}=0.63$ of Brown et al. Arjomandy et al. (Arjomandy et al., 2012) measured depth doses in water at $75 \mathrm{kV}$. Dose response calibration was performed at $75 \mathrm{kV}$. Compared to measurements with an ion chamber, the film showed an over-response of $8-9 \%$ at a depth of $10 \mathrm{~cm}$ compared to the normalization depth of $2 \mathrm{~cm}$. The effective energy was noted to increase from $32.1 \mathrm{keV}$ to $38.4 \mathrm{keV}$. Only $3 \%$ of this increase can be assigned to an increase in the energy absorption of the film relative to water indicating an increase in relative intrinsic efficiency consistent with the results of Brown et al. An increase in effective energy of only $1 \mathrm{keV}$, from $34 \mathrm{keV}$ to 35 $\mathrm{keV}$ in our EBS measurements at $50 \mathrm{~mm}$ compared to $30 \mathrm{~mm}$ is not likely to have a noticeable effect on the results. It may be of some interest here to note that Kirby et al. (Kirby et al., 2010) noted an under-response of 0.6 for the EBT2 film in the Bragg-peak of protons and suggested a model of explanation similar to those used in explaining the relative intrinsic efficiency of low energy photons relative to MV photon beams for TLDs and EPR dosimeters.

\section{Conclusions}

This work is a contribution to developing reliable experimental methods suitable for dosimetry around low energy brachytherapy sources, in particular high dose rate EBS. The EPR dosimeters were found to yield results in agreement with Monte Carlo simulations at three distances $(10,30$ and $50 \mathrm{~mm})$ from the source in the phantom. The EBT2 films agreed with Monte Carlo and EPR at $30 \mathrm{~mm}$ and $50 \mathrm{~mm}$. At $10 \mathrm{~mm}$, the uncertainty in the exact positioning of the EBS was too large to get a reliable estimate using EBT2 films. This work has shown the complexity of measurements around this type of source and the importance of detailed knowledge of the properties of the dosimetry system used. It was also shown that lithium formate EPR dosimetry is a good candidate for the purpose and it was deduced that there might be issues regarding energy dependence and intrinsic efficiency of the EBT2 film that need to be considered in future measurements.

\section{Acknowledgements}

This work was supported by the Swedish Cancer Foundation Grants CAN 2012/764 and CAN 2009/1113 and by Grant \#2011-700810 of the Canadian Cancer Society Research 
Institute (CCSRI). S.W. was funded by MAASTRO-Atrium research grant. G.L. was supported by the Natural Sciences and Engineering Research Council of Canada (NSERC) and by the O'Brien Foundation. B.R. was supported by a Marie Curie Reintegration Grant (Grant Agreement No. PIRG05-GA-2009-247878 from FP7-PEOPLE-2009-RG). H.G. acknowledges The Swedish Research Council (diarienr 2009-5430). Xoft, Inc and ISP are gratefully acknowledged for providing technical details for the simulations and experiments. 


\section{Appendix}

\section{Determination of absorbed dose to water with EPR dosimeters}

Determination of absorbed dose to water using EPR dosimeters in the PMMA phantom at a distance $r$ from the source follows Eq (A1).

$$
\begin{aligned}
& D_{\mathrm{w}, \text { phan }, 50 \mathrm{kV}}(r)= \\
& =M_{50 \mathrm{kV}}(r)\left[\frac{D_{\mathrm{w}}}{M}\left[_{\mathrm{m}} \bar{s}_{\mathrm{col}}\right]_{\mathrm{w}}^{\mathrm{PMMA}} \overline{f_{\mathrm{B}}}\right]_{\mathrm{GMV}} \frac{R_{\mathrm{Q}_{0}}}{R_{\mathrm{Q}}}\left[\frac{\bar{\mu}_{\mathrm{en}}}{\rho}\right]_{\mathrm{det}}^{\mathrm{w}} f_{\mathrm{vol}} \frac{\bar{D}_{\text {det, } \mathrm{MC}}}{\bar{D}_{\text {lithium formate }}}
\end{aligned}
$$

where $M$ denotes the EPR signal, $\left[{ }_{\mathrm{m}} \bar{S}_{\text {col }}\right]_{\mathrm{w}}^{\mathrm{PMMA}}$ is the mass collision stopping power ratio between PMMA and water and converts absorbed dose to water to absorbed dose to PMMA; $\bar{f}_{\mathrm{B}}=\bar{D}_{\text {det }} / D_{\text {PMMA }}$ is the Burlin cavity theory coefficient and converts absorbed dose to PMMA to the mean absorbed dose in the detector. Detailed information and numerical values of these factors are found in Antonovic et al. (Antonovic et al., 2009). $R_{\mathrm{Q}_{0}} / R_{\mathrm{Q}}$ is the correction for intrinsic efficiency (Adolfsson et al., 2010) and was set to 1.06.

The values of $\left[\bar{\mu}_{\mathrm{en}} / \rho\right]_{\mathrm{det}}^{\mathrm{w}}$ were determined to be $1.249,1.246$ and 1.243 at $10 \mathrm{~mm}, 30 \mathrm{~mm}$ and $50 \mathrm{~mm}$ respectively by weighting over the photon energy fluence spectrum at the three measurement positions in the phantom. Mass energy absorption coefficients were taken from NIST (Hubbell and Seltzer, 1995). The volume averaging correction factor, $f_{\text {vol, was }}$ calculated with MC as the quotient of the absorbed dose to a $1 \mathrm{~mm}^{3}$ "point detector" situated at the centre of the EPR dosimeter and the absorbed dose to the full size detector. The values of $f_{\mathrm{vol}}$ at 10,30 and $50 \mathrm{~mm}$ were $1.020,1.023$, and 1.030 respectively. The last factor in Eq A1 is needed to account for the fact that the signal is proportional to the dose registered in the lithium formate grains and not to that registered in the surrounding paraffin. This factor was calculated using MC simulations as described in detail by Adolfsson et al. (Adolfsson et al., 2010).

\section{Determination of absorbed dose to water with EBT2 film}

Determination of absorbed dose to water using the EBT2 film in the PMMA phantom at the distance $r$ from the source followed $\mathrm{Eq}$ (A2)

$$
D_{\mathrm{w}, \mathrm{phan}, 50 \mathrm{kV}}(r)=\frac{M_{\mathrm{phan}(r)}\left[\frac{M}{D_{\mathrm{w}}}\right]_{\mathrm{cal}, 50 \mathrm{kV}}}{\left[\frac{M}{D_{\mathrm{w}}}\right]_{\mathrm{cal}, 50 \mathrm{kV}}\left[\frac{M}{D_{\mathrm{w}}}\right]_{\mathrm{phan}, \mathrm{r}}} f_{\mathrm{pos}} f_{\mathrm{vol}}=\frac{M_{\mathrm{phan}(r)}\left[\frac{\bar{\mu}_{\mathrm{en}}}{\rho}\right]_{\mathrm{w}, \mathrm{cal}, 50 \mathrm{kV}}^{\mathrm{det}}}{\left[\frac{M}{D_{\mathrm{w}}}\right]_{\mathrm{cal}, 50 \mathrm{kV}}\left[\frac{\bar{\mu}_{\mathrm{en}}}{\rho}\right]_{\mathrm{w}, \mathrm{phan}, \mathrm{r}}^{\mathrm{det}}} \frac{R_{\mathrm{Q}_{0}}}{R_{\mathrm{Q}}} f_{\mathrm{pos}} f_{\mathrm{vol}}
$$


The film was calibrated to measure air kerma free-in-air using the TG61 protocol (Ma et al., 2001). Absorbed dose to water was calculated by multiplying air kerma with the ratio of the weighted mean of the mass energy absorption coefficients for water and air yielding water kerma, i.e., absorbed dose to water under charged particle equilibrium. The quotient $\left[\bar{\mu}_{\mathrm{en}} / \rho\right]_{\mathrm{w}, \mathrm{cal}, 50 \mathrm{kV}}^{\mathrm{det}} /\left[\bar{\mu}_{\mathrm{en}} / \rho\right]_{\mathrm{w}, \mathrm{phan}, r}^{\mathrm{det}}$ was set to unity. A correction for film position in the phantom $f_{\text {pos }}$ was derived by $\mathrm{MC}$ calculation of the absorbed dose to water in the volume occupied by the film in the experiment and the same volume positioned at the centre of the EPR dosimeters (in the absence of the EPR dosimeter). Numerical values at 30 and $50 \mathrm{~mm}$ were 1.020 and 1.030 respectively. A volume averaging factor $f_{\text {vol }}$ for the film was applied, derived similarly to the volume averaging for the EPR dosimeters. The quotient of the absorbed dose to a "film point" $\left(1 \mathrm{~mm}^{3}\right)$ and the absorbed dose to the full size film area was calculated. Numerical values at 30 and $50 \mathrm{~mm}$ were 0.991 and 0.976 respectively.

In the last equality of Eq A2 it is assumed that the signal from the film is proportional to the mean absorbed dose to the detector. The factor $R_{\mathrm{Q}_{0}} / R_{\mathrm{Q}}$ takes into account any deviation from this assumption caused by different LET distributions of the secondary electrons at calibration and in the measurement in the PMMA phantom. Here, $R_{\mathrm{Q}_{0}} / R_{\mathrm{Q}}$ was assumed to be unity.

\section{Uncertainty in the experimentally determined absorbed dose}

The total combined relative standard uncertainties in absorbed dose $u\left(D_{\mathrm{w}, \mathrm{phan}, 50 \mathrm{kV}}(r)\right) / D_{\mathrm{w}, \mathrm{phan}, 50 \mathrm{kV}}(r)$, derived from Eqs (A1) and (A2) were estimated by quadratically adding the relative standard uncertainties of its parameters (JCGM 2008)). Derivation of the uncertainties for the EPR dosimeters was described in detail in (Antonovic et al., 2009; Adolfsson et al., 2010). The estimated uncertainties are presented in Table A1.

Estimates of the relative standard uncertainties related to the film calibration (points 2 and 3 in Table A1) were taken from the AAPM protocol (Ma et al., 2001). The mass-energy absorption correction factor (point 4 in Table A1) was set to unity. However, for a film with the composition of the active layer in Table 1 (the film used in this work), corrections of 0.975 and 0.966 at $30 \mathrm{~mm}$ and $50 \mathrm{~mm}$, respectively, should be applied. This correction was not introduced in calculating $D_{\mathrm{w}, \mathrm{phan}, 50 \mathrm{kV}}(r)$ since it cannot be ascertained that the atomic composition is exactly that aimed at by the manufacturer. Instead, we used the information in Figure 1 to estimate uncertainties caused by neglecting the correction. Assuming maximum possible deviations of $2.5 \%(30 \mathrm{~mm})$ and $3.4 \%(50 \mathrm{~mm})$ from unity for this factor, standard uncertainties were obtained by dividing with $\sqrt{3}$ (assuming a triangular probability distribution).

The uncertainty in MC calculated doses depend on the statistical uncertainty in the MC simulations, the uncertainty in the film dose at the normalization depth $(30 \mathrm{~mm})$ and a correction to account for the different positions of the Markus chamber in the experiment 
used for normalization (perpendicular to the beam axis) and in the experiment itself (see Figure 2). The combined relative standard uncertainty was estimated to be $3.5 \%$.

Table A1. Components contributing to the combined relative standard uncertainty for EPR dosimeters and EBT2 film.

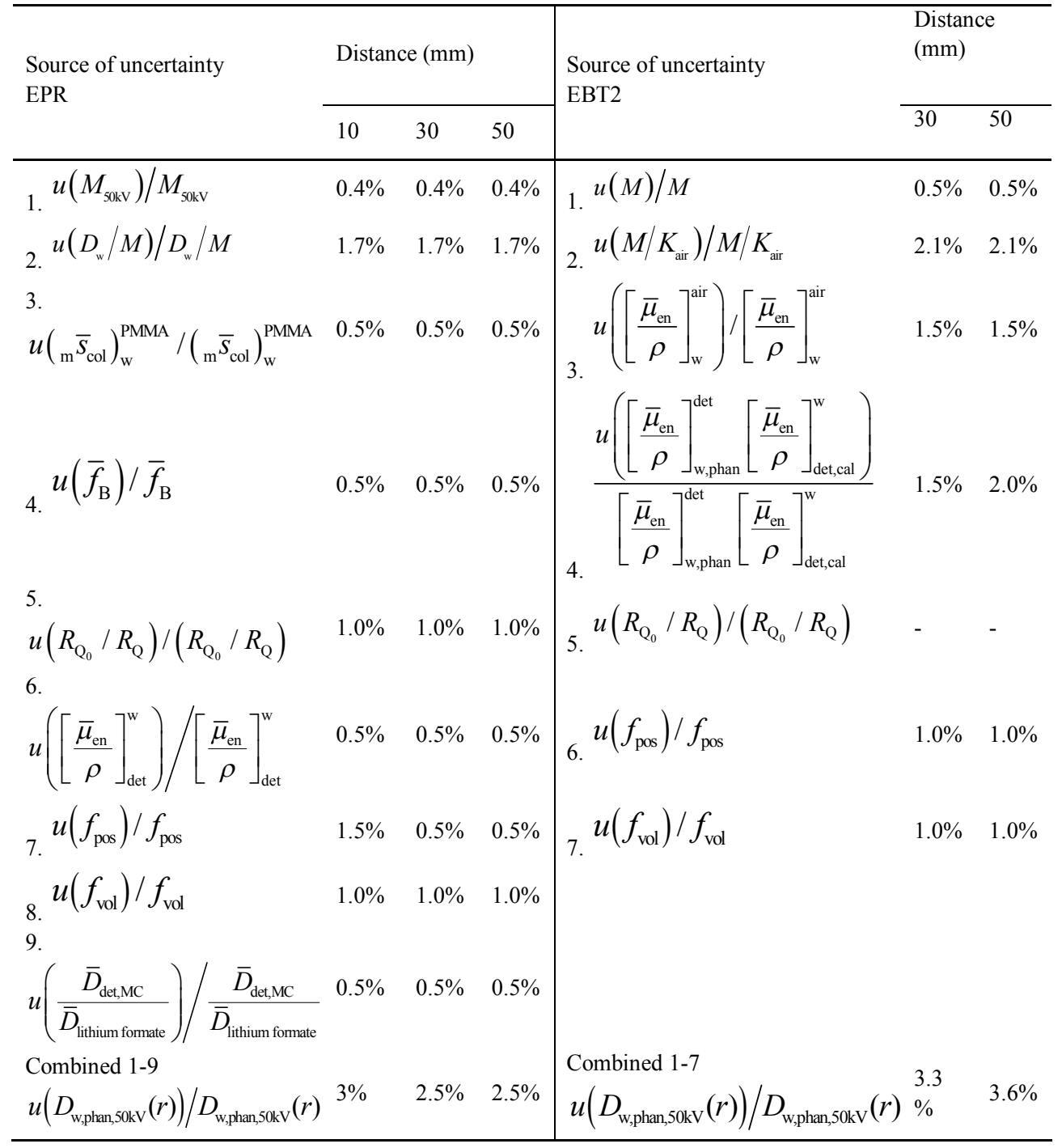




\section{References}

Adolfsson E, Alm Carlsson G, Grindborg J-E, et al. 2010 Response of lithium formate EPR dosimeters at photon energies relevant to the dosimetry of brachytherapy Medical Physics 37 (9) 4946-59

Adolfsson E, Carlsson Tedgren A, Alm Carlsson G, Gustafsson H and Lund E 2014 Optimisation of an EPR dosimetry system for robust and high precision dosimetry Radiation Measurements 70 21-8

Adolfsson E, Karlsson M, Alm Carlsson G, et al. 2012 Investigation of signal fading in lithium formate EPR dosimeters using a new sensitive method Phys. Med. Biol. 57 2209-17

Agostinelli S, Allison J, Amako K, et al. 2003 GEANT4 - A simulation toolkit Nuclear Instruments and Methods in Physics Research, Section A: Accelerators, Spectrometers, Detectors and Associated Equipment 506 250-303

Almond P R, Biggs P J, Coursey B M, et al. 1999 AAPM's TG-51 protocol for clinical reference dosimetry of high-energy photon and electron beams Med. Phys. 26 1847-70

Andreo P, Burns D T, Hohlfeld K, et al. 2000 Absorbed Dose Determination in External Beam Radiotherapy, IAEA TRS 398: An International Code of Practice for Dosimetry Based on Standards of Absorbed Dose to Water. In: IAEA Technical Report Series no 398 (Vienna: International Atomic Energy Agency), (Vienna, Austria: IAEA)

Antonovic L, Gustafsson H, Alm Carlsson G and Carlsson Tedgren $\AA 2009$ Evaluation of a lithium formate EPR dosimetry system for dose measurements around ${ }^{192}$ Ir brachytherapy sources Med. Phys. 36 223647

Arjomandy B, Tailor R, Zhao L and Devic S 2012 EBT2 film as a depth-dose measurement tool for radiotherapy beams over a wide range of energies and modalities Medical Physics 39 912-21

Brown T A D, Hogstrom K R, Alvarez D, et al. 2012 Dose-response curve of EBT, EBT2, and EBT3 radiochromic films to synchrotron-produced monochromatic x-ray beams Medical Physics 39 7412-7

Butson M J, Yu P K N, Cheung T and Alnawaf H 2010 Energy response of the new EBT2 Radiochromic film to $\mathrm{X}$-ray radiation Radiation Measurements 45 836-9

Carlsson Tedgren A, Hedman A, Grindborg J-E and Carlsson G A 2011 Response of LiF:Mg,Ti thermoluminescent dosimeters at photon energies relevant to the dosimetry of brachytherapy (\&lt;1 MeV) Medical Physics 38 5539-50

Cullen D, Hubbell J H and Kissel L 1997 EPDL97: the Evaluated Photon Data Library, 97 version UCRL-50400 Vol. 6, Rev 5

Davis S D, Ross C K, Mobit P N, et al. 2003 The response of LiF thermoluminescence dosimeters to photon beams in the energy range from $30 \mathrm{kV}$ X rays to ${ }^{60} \mathrm{Co}$ gamma rays Radiat. Prot. Dosim. $10633-43$

Granton P V, Podesta M, Landry G, et al. 2012 A combined dose calculation and verification method for a small animal precision irradiator based on onboard imaging Medical Physics 39 4155-66

Gustafsson H, Lund E and Olsson S 2008 Lithium Formate EPR Dosimetry for verification of calculated dose distributions prior to intensity modulated radiation therapy Phys. Med. Biol. 53 4667-82

Hubbell J H and Seltzer S M 1995 Tables of x-ray mass attenuation coefficients and mass energy-absorption coefficients $1 \mathrm{keV}$ to $20 \mathrm{MeV}$ for elements $\mathrm{Z}=1$ to 92 and 48 additional substances of dosimetric interest. (Washington DC, USA: NIST)

International Organization for Standardization I 1995 Guide to the expression of uncertainites in measurements.

Kirby D, Green S, Palmans H, et al. 2010 LET dependence of GafChromic films and ion chamber in low-energy proton dosimetry Phys. Med. Biol. 55 417-33

Lindsay P, Rink A, Ruschin M and Jaffray D 2010 Investigation of energy dependence of EBT and EBT-2 Gafchromic film Medical Physics 37 571-6

Ma C-M, Coffey C W, DeWerd L A, et al. 2001 AAPM protocol for 40-300 kV x-ray beam dosimetry in radiotherapy and radiobiology Medical Physics 28 868-93

Micke A, Lewis D F and Yu X 2011 Multichannel film dosimetry with nonuniformity correction Medical Physics 38 2523-34 
Mille M M, Xu X G and Rivard M J 2010 Comparison of organ doses for patients undergoing balloon brachytherapy of the breast with HDR I192r or electronic sources using Monte Carlo simulations in a heterogeneous human phantoma) Medical Physics 37 662-71

Nunn A A, Davis S D, Micka J A and DeWerd L A 2008 LiF:Mg,Ti TLD response as a function of photon energy for moderately filtered $\mathrm{x}$-ray spectra in the range of $20--250 \mathrm{kVp}$ relative to [sup 60]Co Medical Physics 35 1859-69

Perkins S T, Cullen D E, Chen M H, et al. 1997 Table and graphs of atomic subshell and relaxation data derived from the LLNL Evaluated Atomic Data Library (EADL) UCRL-50400 Vol. 30

Rivard M J, Davis S D, DeWerd L A, Rusch T W and Axelrod S 2006 Calculated and measured brachytherapy dosimetry parameters in water for the Xoft Axxent X-Ray Source: An electronic brachytherapy source Medical Physics 33 4020-32

Sutherland J G H and Rogers D W O 2010 Monte Carlo calculated absorbed-dose energy dependence of EBT and EBT2 film Medical Physics 37 1110-6

van Hoof S, Granton P, Landry G, Podesta M and Verhaegen F 2012 Evaluation of a novel triple-channel radiochromic film analysis procedure using EBT2 Physics in Medicine and Biology 57 4353-68

White S A, Landry G, Fonseca G P, et al. 2014 Comparison of TG-43 and TG-186 in breast irradiation using a low energy electronic brachytherapy source Medical Physics 41, 061701 



\section{Chapter 5:}

\section{A comparison of the relative biological effectiveness of low energy electronic brachytherapy sources in breast tissue: A Monte Carlo study.}

Shane A. White, Brigitte Reniers, Evelyn de Jong, Thomas Rusch, Frank Verhaegen 


\begin{abstract}
Purpose

Electronic brachytherapy sources use low energy photons to treat the tumor bed during or after breast-conserving surgery. The relative biological effectiveness of two electronic brachytherapy sources was explored to determine if spectral differences due to source design influenced radiation quality and if radiation quality decreased with distance in the breast.
\end{abstract}

\title{
Method and Materials
}

The RBE was calculated through the number of DNA double strand breaks ( $\mathrm{RBE}_{\mathrm{DSB}}$ ) using the Monte Carlo Damage Simulator (MCDS) in combination with other Monte Carlo electron/photon spectrum calculations. $50 \mathrm{kVp}$ photons from the Intrabeam (Carl Zeiss Surgical) and Axxent (Xoft) through 40-mm spherical applicators were simulated to account for applicator and tissue attenuation in a variety of breast tissue compositions. $40 \mathrm{kVp}$ Axxent photons were also simulated. Secondary electrons (known to be responsible for most DNA damage) spectra at different distance were inputted into MCDS to calculate the RBE $\mathrm{DSB}_{\text {. All }}$ $\mathrm{RBE}_{\mathrm{DSB}}$ used a cobalt-60 reference. $\mathrm{RBE}_{\mathrm{DSB}}$ data was combined with corresponding average photon spectrum energy for the Axxent and applied to model-based average photon energy distributions to produce an $\mathrm{RBE}_{\mathrm{DSB}}$ map of an accelerated partial breast irradiation (APBI) patient.

\section{Results and Discussion}

Both Axxent and Intrabeam $50 \mathrm{kVp}$ spectra were shown to have a comparable $\mathrm{RBE}_{\mathrm{DSB}}$ of between 1.4 and 1.6 at all distances in spite of progressive beam hardening. The Axxent $40 \mathrm{kVp}$ also demonstrated a similar $\mathrm{RBE}_{\mathrm{DSB}}$ at distances. Most $\mathrm{RBE}_{\mathrm{DSB}}$ variability was dependent on the tissue type as was seen in $\mathrm{rib}\left(\mathrm{RBE}_{\mathrm{DSB}} \approx 1.4\right)$, gland $(\approx 1.55)$, adipose $(\approx$ $1.59)$, skin $(\approx 1.52)$ and lung $(\approx 1.50)$. $\mathrm{RBE}_{\mathrm{DSB}}$ variability between both sources was within $2 \%$. A correlation was shown between $\mathrm{RBE}_{\mathrm{DSB}}$ and average photon energy and used to produce an $\mathrm{RBE}_{\mathrm{DSB}}$ map of a dose distribution in an APBI patient dataset.

\section{Conclusion}

Radiation quality is very similar between electronic brachytherapy sources studied. No significant reductions in $\mathrm{RBE}_{\mathrm{DSB}}$ were observed with increasing distance from the source. 


\section{Introduction}

Electronic Brachytherapy (eBt) is a form of radiotherapy that utilizes low energy photon emission to treat cancer. Low energy brachytherapy has advantages in terms of limiting dose to organs at risk while maximizing the dose to clinically relevant regions due to the higher attenuation/absorption of low energy photons in matter. One notable advantage is the higher linear energy transfer (LET) of secondary electrons generated by low energy photons (Reniers and Verhaegen, 2006). Low energy electrons transfer their kinetic energy to the surrounding matter with a higher ionization density than electrons generated by high energy photons. The higher LET induces a greater level of damage in tissue and, as such, has a greater relative biological impact than a higher energy photon source. The relative biological effectiveness (RBE) is a ratio of the doses for two different radiation sources that yield the same biological impact, such as the surviving cell fraction. RBE is a complex quantity determined by biological endpoint, dose-rate, total dose, fractionation scheme and also the radiation quality/LET (Nikjoo and Lindborg, 2010). Higher RBE is indicative of greater biological damage, where the dose deposited by a source causes a larger amount of clinically useful tumour damage and/or greater organ complications. Currently, the International Commission on Radiation Protection assigns a radiation weighting factor of unity for all photon emitting sources (ICRP, 2007), equating the biological effectiveness of high and low energy photon sources.

One endpoint for determining RBE is the number of radiation-induced DNA strand breaks (Semenenko and Stewart, 2004). DNA damage can be expressed in terms of double strand breaks (DSB) and single strand breaks (SSB) to the DNA. SSB tend to correlate poorly with biological effectiveness while DSB and clusters of DSB have been postulated to lead to greater DNA damage (Goodhead, 1994) (Nikjoo and Lindborg, 2010). The RBE for SSB or DSB induction can be calculated by:

$$
\mathrm{RBE}=\frac{(\text { Strand break yield })_{\text {Reference }}}{(\text { Strand break yield })}
$$

Where 'strand break yield' refers to DSB or SSB yields.

A previous study had estimated the RBE of a $50 \mathrm{kV}$ eBt source (Axxent S700, Xoft Inc, CA) in a variety of tissues, reporting an enhanced $\mathrm{RBE}_{\mathrm{DSB}}$ compared to reference Co-60 photons (Reniers et al., 2008). The RBE ${ }_{\mathrm{DSB}}$ was estimated to be about 1.5 in breast tissue and about $1.1-1.2$ in bone. However, this study used only non-clinical simplified phantom geometries with uniform tissues and ignored the effects of the Axxent balloon applicator, clinical breast tissue mixtures (adipose and gland mixtures) and the variation of RBE with distance from the source.

In the present study the $\mathrm{RBE}_{\mathrm{DSB}}$ for the $50 \mathrm{kVp}$ Axxent $\mathrm{S} 700$ (Xoft, San Jose CA) and the Intrabeam (Carl Zeiss Surgical, Oberkochen, Germany) were calculated using MC simulations. MC-based photon spectral data for the Axxent and Intrabeam sources was used from model x-ray tube simulations(Watson and Seuntjens, 2014; Liu et al., 2008). The differences in radiation quality between $40 \mathrm{kVp}$ and $50 \mathrm{kVp}$ modes of the Axxent were also explored, taking into account applicators and tissues of different thickness and composition. 
The $40 \mathrm{kVp}$ mode provides steeper dose distributions and may lead to even higher RBE values (Mestres et al., 2008), but this is currently clinically unexplored. The difference in radiation quality was also investigated between both eBt sources both operated at $50 \mathrm{kVp}$, but with a different design leading to differences in emitted photon spectra.

\section{Methods and Materials}

\section{Electronic Brachytherapy Sources}

\section{Intrabeam}

The Intrabeam source is a low energy drift-tube eBt source, approximately $3 \mathrm{~mm}$ in diameter and $10 \mathrm{~cm}$ in length (Elliott et al., 2011). The Intrabeam source can be used in single fraction partial breast irradiation as an alternative to external beam breast irradiation for carefully selected patients (Kraus-Tiefenbacher et al., 2005) (Deneve et al., 2012) (Eaton et al., 2012).(Elliott et al., 2011; Sanders et al., 2007). IORT presents many advantages over external beam radiotherapy and has been the subject of an extensive 10 year randomized control trial, TARGIT (Vaidya et al., 2001). The Intrabeam source utilizes a $50 \mathrm{keV}$ beam of electrons directed through the drift tube to a thin gold transmission target, located at the tip, to generate a bremsstrahlung x-ray spectrum (Figure 1). The electron accelerator section of the Intrabeam source is located in part of the Intrabeam exterior to the patient. The Intrabeam source can be used with various sizes of spherical solid applicators made of polyetherimide (PEI), with diameters ranging from $1.5-50 \mathrm{~mm}$. Photon spectra for specific applicators were not available. To determine the spectral attenuation due to applicator/tissue attenuation, a calculated photon spectrum from the Intrabeam source, obtained from an EGSnrc-based MC model of the source, was used in this study as the initial spectrum (Watson and Seuntjens, 2014). The calculated half value layers of the bare probe and various spherical applicators were in close agreement with measured data from literature. The Intrabeam produces a continuous bremsstrahlung spectrum with a low average energy $(13.7 \mathrm{keV})$ that is dominated at low energies by gold K-edge characteristic x-rays. These are rapidly attenuated by the surrounding applicator (Watson and Seuntjens, 2014) In this study, only the 40mm PEI spherical applicator was modeled with uniform composition and density.

\section{Axxent source}

The Axxent eBt source is a miniature $\mathrm{x}$-ray tube approximately $2.3 \mathrm{~mm}$ in diameter and $1.5 \mathrm{~cm}$ in length. Electrons generated in the tip are accelerated through a $50 \mathrm{kV}$ potential (Liu et al., 2008) after which they strike a tungsten transmission anode target (Figure 1). The anode also contains yttrium for added material strength and the source contains silver for conductivity. Characteristic X-ray peaks are generated at the tungsten L-edge, yttrium and silver K-edges between $9.7 \mathrm{keV}$ and $22.3 \mathrm{keV}$ with a continuous bremsstrahlung photon spectrum up to $50 \mathrm{keV}$ with an average energy of $25.3 \mathrm{keV}$. The $40 \mathrm{kVp}$ Axxent spectrum also comprises these characteristic photons and has a slightly lower average energy $(22.2 \mathrm{keV})$. The target material (gold vs. tungsten) is the main difference between the two eBt sources studied (Intrabeam vs. 
Axxent). Much of the low energy photon spectrum is attenuated by a water-cooling sheath around the anode. The MC-calculated Axxent spectrum used in this study has been validated against measured data (Liu et al., 2008).

The Axxent applicator consists of a saline-inflated silicone balloon with a hollow air channel to accommodate the Axxent source at varying dwell positions. The applicator size ranges in diameter from $30 \mathrm{~mm}$ to $60 \mathrm{~mm}$ though the actual size depends on the amount of saline injected. The balloon applicator surface is composed of an outer sheath (thickness $\sim 0.3$ $0.5 \mathrm{~mm}$ ) of silicone and barium sulphate, a CT contrast material that enhances imaging contrast between the balloon applicator surface and the surrounding planning target volume (PTV) (Segala et al., 2011). Barium is a high-Z element that strongly attenuates photons with energies at and above the binding energy of its $\mathrm{K}$-edge $(37.4 \mathrm{keV})$. The lack of attenuation in the air channel for forward-emitted photons is compensated for by a high- $Z$ silicone tip (Figure 1).

The Axxent applicator was modeled as a $40 \mathrm{~mm}$ diameter sphere with a $0.4 \mathrm{~mm}$ thick bariumimpregnated silicone wall. The applicator diameter of the Axxent and the Intrabeam were identical.
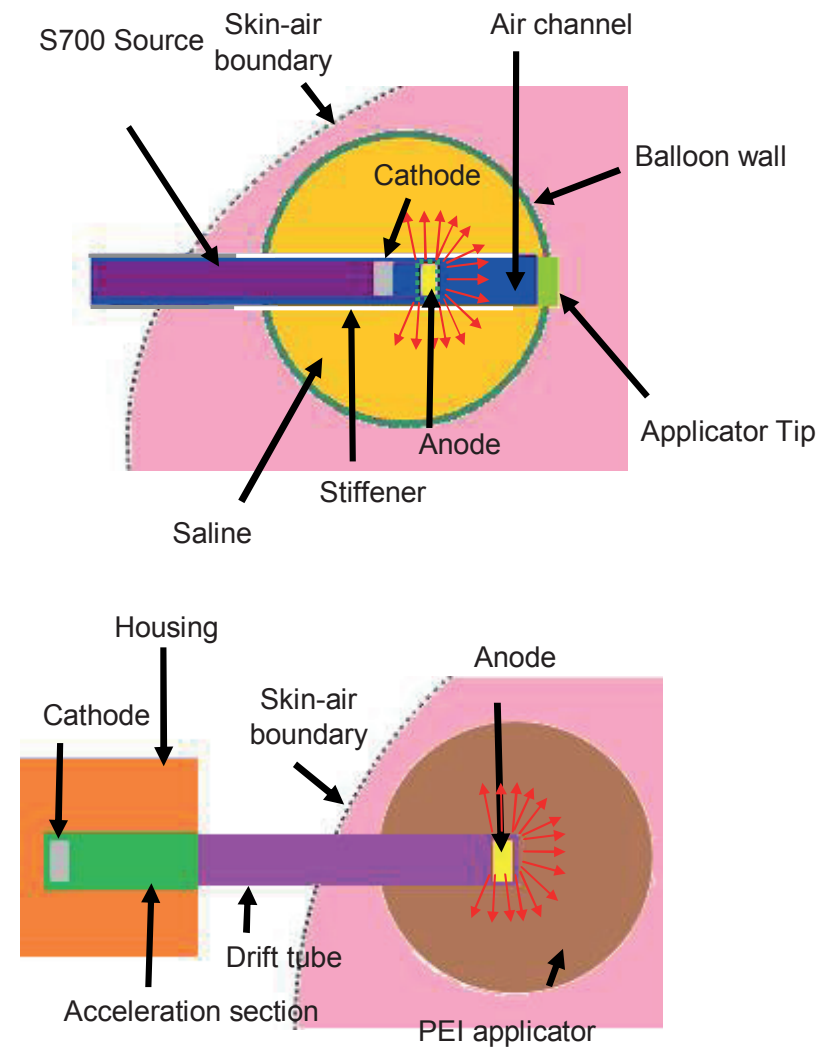

Figure 1: A schematic drawing (top) of the Axxent balloon applicator with the S700 source. The balloon wall varies in thickness between $0.3 \mathrm{~mm}$ and $0.6 \mathrm{~mm}$. The Intrabeam applicator (bottom) consists of a simpler plastic applicator around the needle-like source (drawings not to scale). 


\section{Electronic brachytherapy photon spectra}

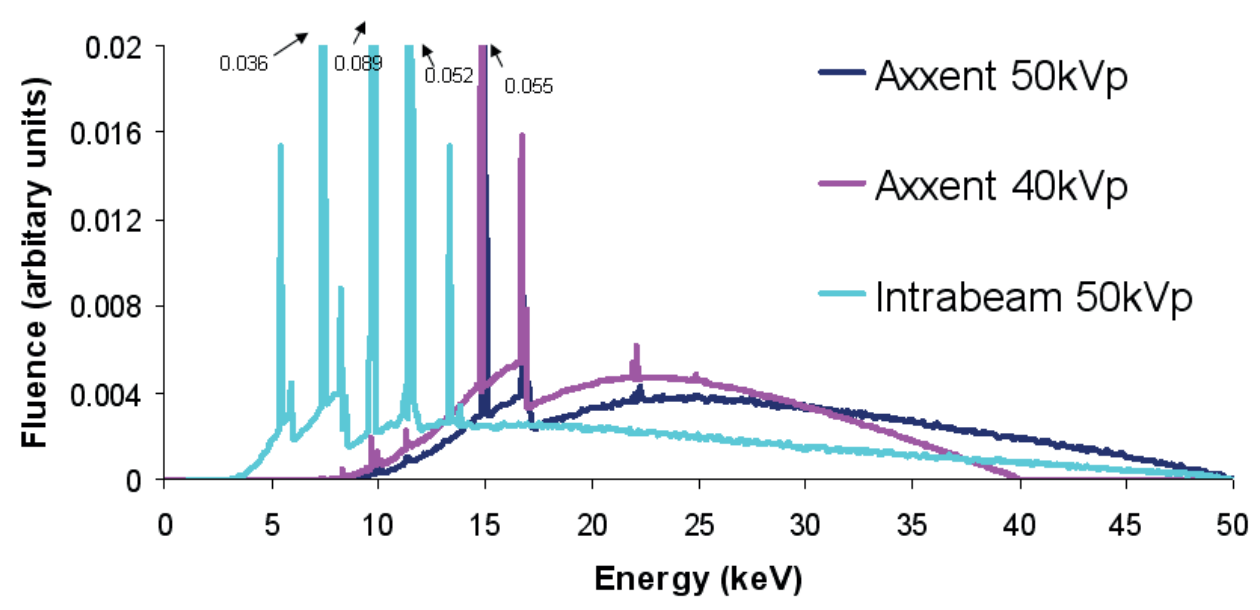

Figure 2: The calculated photon spectra from the surface of the Axxent $(50 \mathrm{kVp}$ and $40 \mathrm{kVp})$ and Intrabeam eBt sources. Simulated anode x-ray production (characteristic photons and bremsstrahlung) for both sources was calculated ( (Watson and Seuntjens, 2014; Liu et al., 2008))prior to the simulations described in this paper. Characteristic x-ray peaks are prominent in all spectra and lower the average photon energy. Note that these photons are rapidly attenuated by the source applicator.

Monte Carlo codes Geant4, EGSnrc and MCDS

Three MC tools were used in this study to determine the RBE for both sources and geometries. Geant4 (Agostinelli et al., 2003) was used to determine photon attenuation around the applicators in the tissues. EGSnrc (Kawrakow, 2000) was used to calculate the secondary electron spectrum that each attenuated photon spectrum generated around the sources and Monte Carlo Damage Simulator (MCDS) (Stewart et al., 2011) was used to calculate the degree of DNA damage for the inputted electron spectrum. All MC programs were used sequentially for each source/kVp (Figure 3 ). Simulations were performed in simple spherical geometries (Figure 4) and complex CT-based patient geometries (Figure 5) in Geant4 . Geant4 was used for the initial photon simulations due to prior experience with CTbased patient geometries with low energy sources. EGSnrc was used for the electron production segment of the workflow. EGSnrc is considered a benchmark for electron simulation problems (Poon and Verhaegen, 2005; Maigne et al., 2011). While the electron spectra could have been calculated using Geant4 in this work, EGSnrc was used for this task for consistency with previous work (Reniers et al., 2008).

Photons from three photon emission spectra (Figure 2) were sampled and scored at different distances from the surface of their respective applicators. The photon/electron spectra were scored in water, adipose, glandular, lung, rib or skin geometries (table 1). Tissue assignment in the CT-based geometries used tissue segmentation algorithm (Afsharpour, 2011)to reflect 
the complex distribution of tissues in the breast. The RBE $\mathrm{DSB}_{\mathrm{DS}}$ was calculated for CT-based geometries using a calculated $\mathrm{RBE}_{\mathrm{DSB}} /$ Average photon energy relationship (see 2.5).

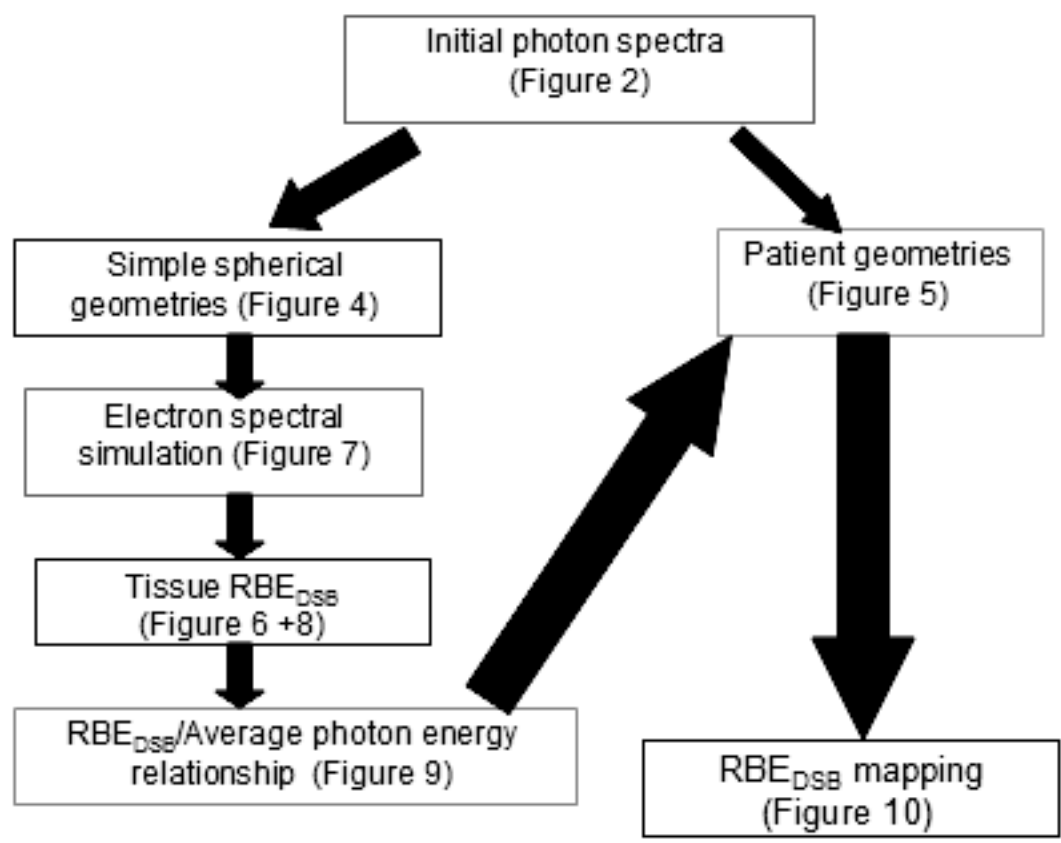

Figure 3: A flowchart demonstrating the workflow from the initial spectra to $\mathrm{RBE}_{\mathrm{DSB}}$ mapping.

\section{Geant4}

The software package used for the photon simulations in this study was the MC toolkit Geant4 v9.5p02. Geant4 is an all-purpose MC simulation toolkit that provides a diverse set of software components that can be employed in a variety of settings, including the simulation of photon transport from low-energy sources (Agostinelli et al., 2003). These simulations used the Lawrence Livermore National Laboratory low-energy electromagnetic model for Geant4 (G4LowEnergy) which simulates the low-energy photon interactions of the photoelectric effect, Compton scattering and Rayleigh scattering. The model uses the EPDLevaluated (Cullen D, 1997) and EADL-evaluated (Perkins, 1997) libraries. The photon energy transport cutoff was $1 \mathrm{keV}$. Geant 4 was used to model the applicator for both sources embedded in a shell of variable thickness (Figure 4) and composed of a component of breast tissue (table 1). Photon transport was simulated using Geant4 and scored in a vacuum shell geometry. Electron production was ignored at this stage as the primary electrons would be calculated separately using another MC code as described in the previous section. All Geant4 simulations were run for $40 \mathrm{M}$ histories to give a $<0.1 \%$ statistical uncertainty for low attenuating tissues close to the applicator, and $1-2 \%$ for highly attenuating rib at $40 \mathrm{~mm}$ from the surface of the applicator. 


\section{EGSnrc}

For the spherical simulation geometry that generated photon spectra in Geant4, its subsequent primary electron spectrum was calculated using EGSnrc (Kawrakow, 2000), a general purpose $\mathrm{C}++$-based Monte Carlo code.

Each electron spectrum was determined using a technique described in a previous study (Reniers et al., 2008). Here, the electron spectrum was scored by simulating interactions from the photon spectrum attenuated through the applicator/tissue material in the previous section Primary electrons, produced from the photo-electric effect, bound Compton scattering and the Auger relaxation process, were scored between two concentric spheres close to the photon source (simulated as a point source). Compton and photo-electrons were generated down to a cutoff energy of $0.1 \mathrm{keV}$ while Auger electrons were produced down to a cutoff of $1 \mathrm{keV}$, (sub-1 keV Auger electrons are irretrievably discarded by EGSnrc). Only primary electrons were produced and scored while secondary electrons were ignored. It should be noted that the cutoff for Auger electrons was insufficient to accurately model the electron spectra for low energy photon spectra in EGSnrc, requiring a manual correction for sub-keV Augers. Calculating the proportion of Auger electrons in the electron spectrum is important due to their relatively high LET.. 100 million histories were simulated to reduce the statistical uncertainty to less than $0.1 \%$.

The primary tissue components which produce Auger electron transitions are the elements C, $\mathrm{N}$ and $\mathrm{O}$, which yield electrons at around $280 \mathrm{eV}, 373 \mathrm{eV}$ and $502 \mathrm{eV}$, respectively. As EGSnrc does not calculate Auger electrons of these energies, it was necessary to estimate the Auger contribution to the total electron spectra. The number of Auger electrons generated was estimated by the total number of photo-electrons scaled according to the photoelectric crosssection of $\mathrm{C}, \mathrm{N}$ or $\mathrm{O}$ (Berger et al., 2005), the fluorescent yield ( $\approx 0$ for low-Z elements) and the mass-fraction of the element in the tissue/material. Auger electrons are also generated by vacancies created by $\mathrm{K}$-shell electron interaction in Compton photon scattering. The crosssection for the K-shell electron in Compton scattering is dependent on the energy of the incident photon (Stutz, 2014). Estimates for the number of K-shell vacancies (and subsequent Auger electrons) were obtained using cross-section data (Stutz, 2014).

\section{Monte Carlo Damage Simulator}

The Monte Carlo Damage Simulator (MCDS) (Semenenko and Stewart, 2004) is a simple and fast MC-based program to simulate the formation of DNA damage by different types of radiation. MCDS presents a faster alternative to microdosimetry $\mathrm{MC}$ simulations for detailed DNA models (Nikjoo et al., 2002) which are time-consuming. The number of DSB are calculated based on the incident primary electron spectrum on the edge of a single cell model with a fixed nucleus diameter $(5 \mu \mathrm{m})$. Damage due to secondary electrons due to slowingdown of primary electrons is accounted for in MCDS. For this study the number of DSB per cell per giga-base-pair was the metric to determine the damage to a cell by a source. DSB are calculated based on the number of lesions within a predetermined number of base-pairs, where two SSB within 8 to 80 base pairs of each other could be considered a DSB (Semenenko and Stewart, 2004). The classification of breaks by complexity has been explored in other studies(Nikjoo et al., 1997) and has been incorporated into MCDS. MCDS also accounts for oxygenation of the cell and the chemical repair that occurs under hypoxic 
condition (Stewart et al., 2011) Hypoxia in cells tends to increase radio-resistance (Georgakilas et al., 2013) and DSB yield under oxygenated and hypoxic conditions has been studied (Carlson et al., 2006) (Whitaker and McMillan, 1992). In this study, two breaks on opposing DNA strands with a separation of less than 10 base pairs (MCDS default) is considered a DSB. In comparison to the classic DNA track structure MC simulations, MCDS agrees to within $1 \%$ for $225 \mathrm{kVp}$, Co-60 and $29 \mathrm{kVp}$ photon sources(Hsiao and Stewart, 2008). DSB yields predicted by MCDS agree to within $7 \%$ for $<2 \mathrm{keV}$ characteristic $\mathrm{x}$-rays in V79 cells described in experimental studies.(Botchway et al., 1997; de Lara et al., 2001).

\section{Variations in photon and electron spectra with depth}

To calculate the $\mathrm{RBE}_{\mathrm{DSB}}$ variation with depth for both eBt sources the change in photon spectra with distance from the applicator was first calculated using Geant 4 by simulating the transport of the photons through a series of tissue geometries of different thicknesses.. With Geant4, a series of concentric shells were used to model all materials (Table 1) individually in the simulated geometry (Figure 4). The outermost shell was composed of a tissue found in/near the breast. Embedded in the tissue shell was a thin scoring shell $(<0.1 \mathrm{~mm})$. The innermost shell was a $40 \mathrm{~mm}$ diameter applicator model. The total thickness of tissue was large $(150 \mathrm{~mm})$ such that it could be considered infinite for the purposes of simulating backscatter. The tissues (Table 1), derived from ICRU-46 (International Commission on Radiation Units and Measurements, 1992), reflected the density and composition of bulk tissue in the breast. A water-based model was also used to model TG-43 conditions.

Table 1: The ICRU 46 tissue compositions for the 5 tissues modeled

Element (\% weight)

\begin{tabular}{l|lllllllllll|l|}
\hline Tissue & $\mathbf{H}$ & $\mathbf{C}$ & $\mathbf{N}$ & $\mathbf{O}$ & $\mathbf{N a}$ & $\mathbf{M g}$ & $\mathbf{P}$ & $\mathbf{S}$ & $\mathbf{C l}$ & $\mathbf{K}$ & $\mathbf{C a}$ & $\begin{array}{l}\text { Density } \\
\left(\mathbf{g} / \mathbf{c m}^{3}\right)\end{array}$ \\
\hline Glandular & 10.6 & 33.2 & 3 & 52.7 & 0.1 & - & 0.1 & 0.2 & 0.1 & - & - & 1.02 \\
\hline Adipose & 11.4 & 59.8 & 0.7 & 27.8 & 0.1 & - & - & 0.1 & 0.1 & - & - & 0.95 \\
\hline Skin & 10 & 20.4 & 4.2 & 64.5 & 0.2 & - & 0.1 & 0.2 & 0.3 & 0.1 & - & 1.09 \\
\hline Rib & 6.4 & 26.3 & 3.9 & 43.6 & 0.1 & 0.1 & 6 & 0.3 & 0.1 & 0.1 & 13.1 & 1.41 \\
\hline Lung & 10.3 & 10.5 & 3.1 & 74.9 & 0.2 & - & 0.2 & 0.3 & 0.3 & 0.2 & - & 0.26 \\
\hline \hline
\end{tabular}




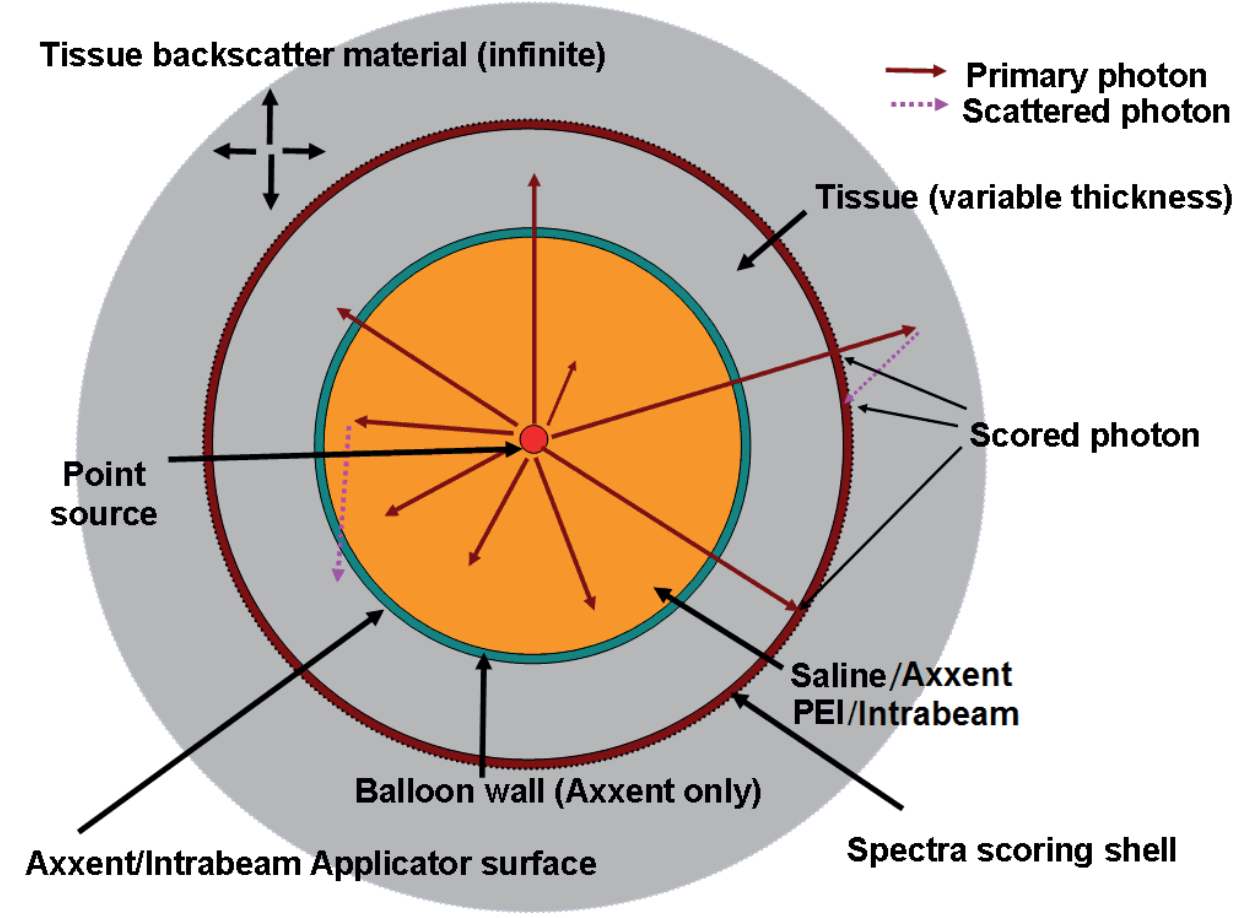

Figure 4: A schematic setup to determine the attenuated photon spectra for the eBt sources using Geant4. The Axxent and Intrabeam applicators are modeled as a $40 \mathrm{~mm}$ sphere of saline/PEI (orange) and a $0.4 \mathrm{~mm}$ balloon wall (green) in the case of the Axxent. Both eBt sources are modelled as isotropic point sources (red dot). The tissue depth (grey) and subsequent spectral scoring shell (dark red) varied from 0 to $40 \mathrm{~mm}$. Full backscatter conditions were included outside the scoring shell and backscattered photons were scored. The setup to determine the electron spectrum using EGSnrc is not shown.

The distance of the scoring shell from the surface of the applicator varied from $0 \mathrm{~mm}$ to $40 \mathrm{~mm}$. The varied distance allows the calculation of $\mathrm{RBE}_{\mathrm{DSB}}$ at the edges of the PTV (0$10 \mathrm{~mm}$ ) (Keisch et al., 2007) and inside normal tissue around the PTV (10-40mm). Photons from the source were generated isotropically from the centre of the applicator. The small anisotropy and source dimensions (Rivard et al., 2006), were not included in this study as this was expected to have only a minor influence on the RBE calculations. As mentioned in previous sections, photons that were transported across the tissue, either un-attenuated or scattered were scored in the spectra scoring shell. Missing photon backscatter from the vacuum region beyond the medium sphere was accounted for. Each photon spectrum was imported into EGSnrc to determine the primary electron spectra with the low energy $\mathrm{C}, \mathrm{N}$ and $\mathrm{O}$ Auger portion of the spectrum for each tissue determined manually using the XCOM data (Berger et $a l, 2005)$.

\section{Calculation of RBE DSB}

The DSB yield was calculated in MCDS for both eBt sources. The biological cells containing the DNA were assumed to be fully oxygen-saturated ( $\mathrm{pO} 2=100 \%$ ) due to the removal of the 
primary (possibly hypoxic) tumour in IORT and APBI patients prior to irradiation. Therefore, primarily normal oxygenated tissue is investigated in this study. DSB yield was simulated in $2.5 \times 10^{5}$ cells to give a statistical uncertainty of $0.2 \%$. The reference DSB yield was calculated for a Co-60 electron spectrum. Derived from transporting $1.25 \mathrm{MeV}$ photons in water in EGSnrc. RBE $\mathrm{DSB}$ was then calculated with Eq1.

\section{$\mathrm{RBE}$ in a realistic $\mathrm{APBI}$ patient geometry}

An $\mathrm{RBE}_{\mathrm{DSB}}$ map was produced accounting for breast tissue heterogeneities, photon and electron spectral changes due to the balloon applicator and tissue attenuation. This map was calculated for a realistic patient geometry derived from a DICOM CT image of an APBI patient treated using the Axxent. In a prior study (White et al., 2014) a CT image was converted into a material/density mapped voxelized phantom (Figure 5) using a tissue contouring method (Afsharpour et al., 2011) and the dose distribution was calculated using Geant4. A similar method could be used to calculate the photon spectra in all the phantom tissue voxels allowing for the modeling of tissue-specific $\mathrm{RBE}_{\mathrm{DSB}}$ distributions similar to proton LET-based maps (Grassberger et al., 2011). The calculation of a $\mathrm{RBE}_{\mathrm{DSB}}$ map presents some challenges. Fast MC DNA damage calculation algorithms are not a feature of Geant4 or EGSnrc, making direct RBE calculations difficult. Calculating the full photon and electron spectrum for each voxel in a patient geometry is also cumbersome due to the sheer number of voxels present in patient images and the time to calculate the $\mathrm{RBE}_{\mathrm{DSB}}$. Calculating the $\mathrm{RBE}_{\mathrm{DSB}}$ in a water-based TG-43 geometry may produce a generic radiation quality model which could be used to determine the change in $\mathrm{RBE}_{\mathrm{DSB}}$ with distance from the source, however x-ray spectra may harden significantly due to changes in tissue composition with low energy photons attenuated to a great degree in high density/atomic number regions such as the ribs. Knowledge of the tissue composition is important for determining the Auger transitions with the carbon, oxygen and nitrogen-rich soft tissues contributing the lowest energy (and highest LET) electrons to a spectrum (Auger electrons with $280 \mathrm{eV}, 373 \mathrm{eV}$ and $502 \mathrm{eV}$, respectively), compared to a phosphorus and calcium-rich $(2 \mathrm{keV}$ and $4 \mathrm{keV})$ tissue such as bone. Thus, tissue heterogeneities and patient geometry might reduce the accuracy of a simple water-based RBE model.

Computational limitations, the volume of spectral data and the large number of voxels in a patient geometry meant that a simpler method of $\mathrm{RBE}_{\mathrm{DSB}}$ determination needed to be developed..
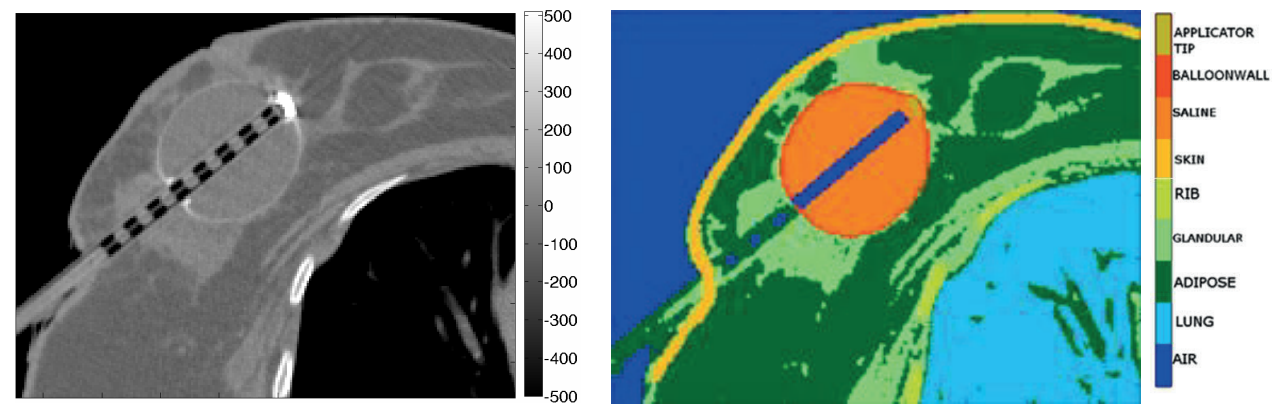
Figure 5: A material/density geometry (right) for a Geant4 simulation with a fully-segmented breast geometry converted from a CT image (left). The balloon applicator is also modeled here to highlight the air channel, saline-filled balloon and balloon tip (appearing as streaking artifacts (left) due to high photon absorption of barium).

In this work, for different breast tissues, simple relationships were derived between the mean photon energy and the $\mathrm{RBE}_{\mathrm{DSB}}$ as a function of distance from an Axxent applicator balloon. These were then applied to mean photon energies calculated for all voxels in the APBI case. Assignment of $\mathrm{RBE}_{\mathrm{DSB}}$ values was based on the material index (table 1). The tissue segmentation assumed no mixed composition voxels. Visualization of the average energy/RBE map was performed using an in-house brachytherapy dose analysis tool, BrachyGUI (Poon et al., 2008).

\section{Results and Discussion}

\section{Axxent vs.Intrabeam : comparison of RBE}

The DSB yield for the reference radiation quality Co-60 was calculated as $8.50 \mathrm{Gbp}^{-1} \mathrm{~Gy}^{-1}$ under normoxic conditions, which is in agreement with those calculated using other Monte Carlo methods (Kirkby et al., 2013) and with experiments, when taking uncertainties into

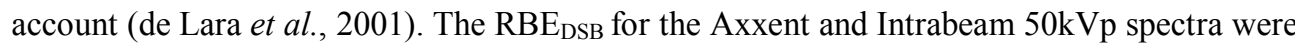
compared at different distances from the surface of their applicators for a variety of tissues. The $\mathrm{RBE}_{\mathrm{DSB}}$ for different tissues was considerably different, ranging between 1.4 and 1.6 at all distances from both sources. The full $\mathrm{RBE}_{\mathrm{DSB}}$ and damage yields are shown in Figure 6 . The difference between the two sources is small and depends slightly on the tissue type: $<1.3 \%$ for adipose, gland, and lung tissue and $<0.5 \%$ for rib.

The $\mathrm{RBE}_{\mathrm{DSB}}$ for both sources did not decrease substantially with distance (Figure 6), in spite of spectral hardening, particularly through rib, where only a small decrease in $\mathrm{RBE}_{\mathrm{DSB}}$ was calculated between $0 \mathrm{~mm}$ and $20 \mathrm{~mm}(<1 \%)$. The absence of a spectral hardening effect on $\mathrm{RBE}_{\mathrm{DSB}}$ and the similarity between both sources would seem to suggest that photons from the lower range of the spectrum $(10-30 \mathrm{keV})$, which are attenuated more readily than those at higher energies, do not somehow contribute to $\mathrm{RBE}_{\mathrm{DSB}}$ significantly or are compensated for by the processes triggered by higher energy photons. Auger electrons generated from the photo-electric and Compton processes are the most influential aspect of the electron spectrum for both sources, contributing approximately $40-50 \%$ of the DSB. When the Auger electrons are excluded, the average $\mathrm{RBE}_{\mathrm{DSB}}$ for both sources decreases to approximately 1.3. As previously shown, Compton and Auger electrons below $8 \mathrm{keV}$ significantly increase the damage to DNA (Reniers et al., 2008). Although a harder photon spectrum would be expected to result in a lower DSB yield, this is not the case as though spectral hardening yields a reduction of photo-electrons and therefore a reduction in photo-electric-generated Auger electrons; (the photoelectric cross-section is higher at lower photon energies), it results in a relative increase in both low-keV Compton electrons and sub-keV Compton-generated Auger electrons which offsets the decrease in DNA damage caused by the harder photon spectrum. It was calculated using the K-shell cross-section (Stutz, 2014) for the photon input 
spectrum for each source that the portion of Auger electrons from Compton to total number of Auger interactions

$$
\left(\frac{\text { Auger }_{\text {Compton }}}{\text { Auger }_{\text {photoelecric+Compton }}}\right)
$$

ranged from $12 \%$ to $32 \%$ (average $21 \%$ ) for the Axxent and $8 \%$ to $30 \%$ (average $17 \%$ ) for the Intrabeam eBt sources depending on the tissue's $\mathrm{C}, \mathrm{N}$, and $\mathrm{O}$ composition. This portion increases with increasing distance.

With respect to previous studies (Reniers et al, 2008, Kellerer, 2002; Brenner and Amols, 1989; Bernal et al., 2011; Kuhne et al., 2005), where the RBE (in terms of physical damage, induction of chromosome aberrations and DSBs in water) for low energy sources (Mammograhy $(29 \mathrm{kVp})$ and characteristic $\mathrm{x}$-rays $(<5 \mathrm{kV})$ ) was calculated to be on the order of 1.15 to 1.3 , the values reported in this study are elevated ( 1.4-1.6) and comparable to another Axxent RBE study (Reniers et al, 2008). Literature values for DSB probabilities (comparable to those calculated using MCDS) more closely match the $\mathrm{RBE}_{\mathrm{DSB}}$ in this study when the Auger component is excluded (1.3).

Detailed, CPU intensive track length Monte Carlo (MC) simulators have modelled DNA strand breakages for photons (Friedland et al., 1999), showing $\mathrm{RBE}_{\mathrm{DSB}}$ on the order of 1.4 to 1.64 for K-edge electrons while RBE for cell deactivation for low energy photons has been quantified experimentally, using V79 cells irradiated with characteristic x-ray (de Lara et al., 2001; Liu et al., 2013) and with the former (de Lara et al, 2001) compared retrospectively with DNA damage simulators with good agreement (Stewart et al., 2011). The more recent of the two studies (Liu et al, 2013) utilized the Intrabeam source to determine clonogenic deactivation in 4 cell types demonstrating an average RBE of 1.3 (Confidance Interval 1.25 1.36). Mammography sources have also been studied and were initially hypothesized to have higher RBE $(\sim 2)$ compared to high energy sources(Brenner and Amols, 1989). Radiation quality has been calculated using other methods. Detailed lineal energy distributions of mammography sources have been calculated using Monte Carlo techniques and shown to produce ionization clusters approximately $35 \%$ higher than $300 \mathrm{kVp}$ photon sources (similar to Ir-192) (Verhaegen and Reniers, 2004)

\section{Axxent Vs. Intrabeam 50kVp}

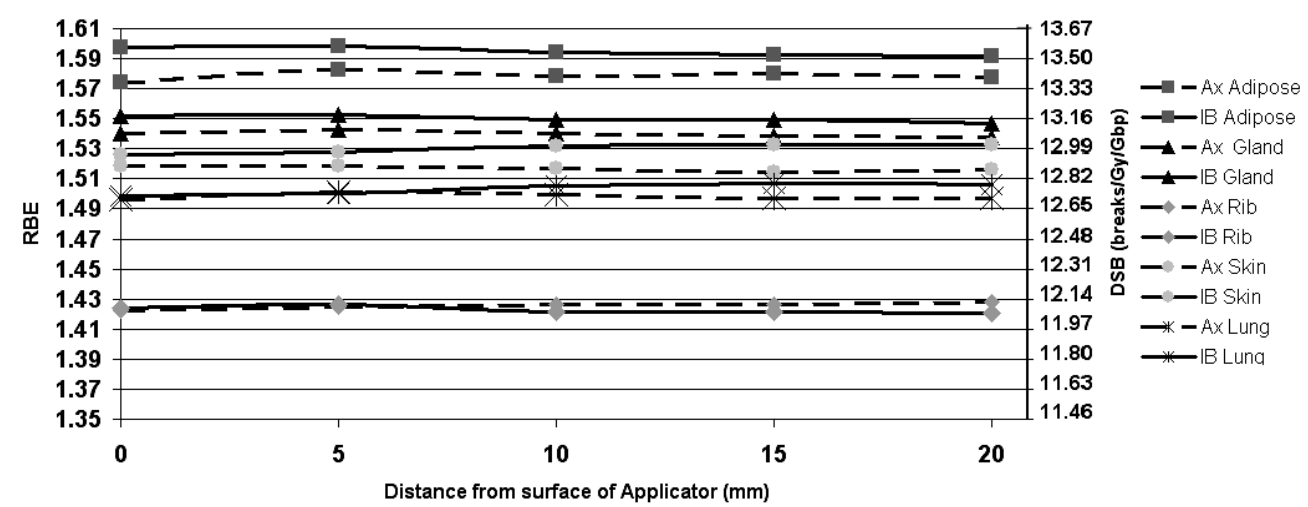

Figure 6: The calculated DSB yield and RBE for the Axxent (Ax) and Intrabeam (IB) 50kVp eBt sources 


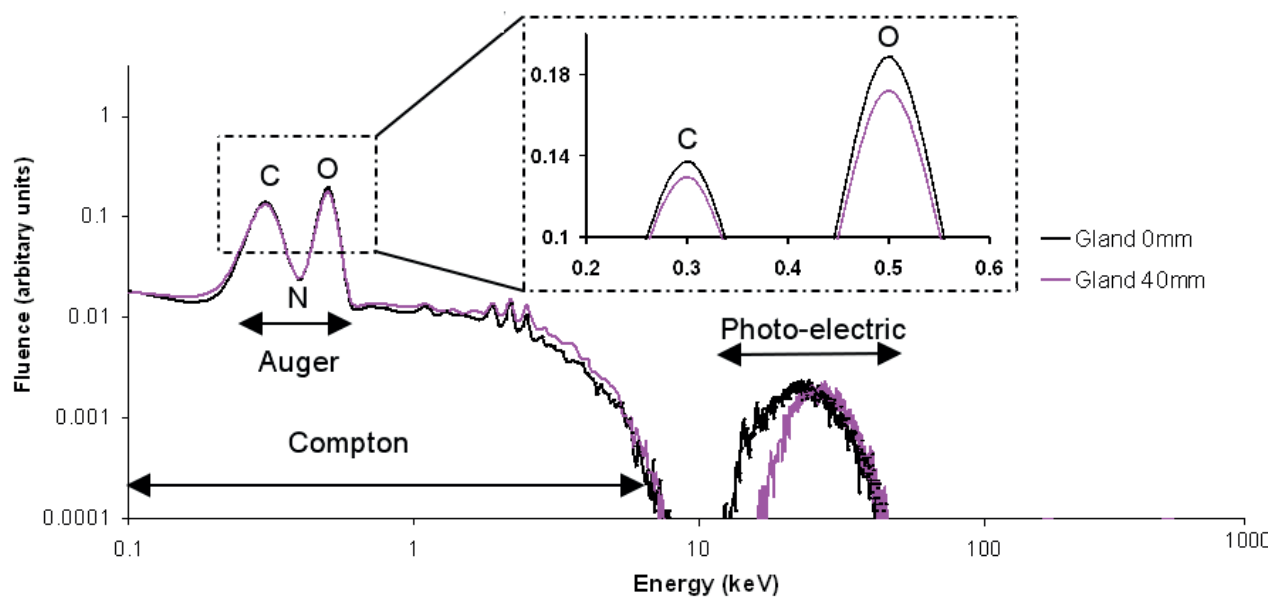

Figure 7: Electron spectra for mammary gland in an Axxent 20mm radius applicator setup at $0 \mathrm{~mm}$ (black) and $40 \mathrm{~mm}$ (pink), normalized against the total fluence. The spectra have distinct regions where Compton and photoelectrons are generated. A decrease in Auger ( 20\%) and photo-electrons and an increase in Compton electrons is observed in the $40 \mathrm{~mm}$ spectra due to spectral hardening. The $\mathrm{N}$ auger peak is relatively small due to the low concentration of nitrogen in gland tissue.

\section{$40 \mathrm{kVp}$ vs. 50kV: Axxent}

A comparison of the $\mathrm{RBE}_{\mathrm{DSB}}$ for the $40 \mathrm{kVp}$ and $50 \mathrm{kVp}$ spectra of the Axxent source was made for a variety of tissues at depths from 0 to $20 \mathrm{~mm}$. The DSB yield and $\mathrm{RBE}_{\mathrm{DSB}}$ for both sources can be seen in Figure 8. For both operating voltages similar RBE $\mathrm{DSB}_{\mathrm{B}}$ were obtained with a small increase in $\mathrm{RBE}_{\mathrm{DSB}}$ for most tissues (average $0.9 \%$ ) for the $40 \mathrm{kVp}$ spectrum. The small increase in $\mathrm{RBE}_{\mathrm{DSB}}$ for the $40 \mathrm{kVp}$ Axxent and Intrabeam spectra described in the previous section, demonstrates the relative $\mathrm{RBE}_{\mathrm{DSB}}$ insensitivity in the $40-50 \mathrm{kVp}$ range to spectral changes. As in the previous section, the reasons for the insensitivity could be due to the offsetting of the effects of spectral hardening by increased Compton electron production. Due to this Compton offsetting, a 'plateau' effect may exist for $30-50 \mathrm{kVp}$ Bremsstrahlung spectra where the $\mathrm{RBE}_{\mathrm{DSB}}$ experiences only small changes. For the tissue with the greatest degree of spectral hardening, rib, the differences in $\mathrm{RBE}_{\mathrm{DSB}}$ between 40 and $50 \mathrm{kVp}$ sources is also negligible, perhaps due to the reduced sub- $1 \mathrm{keV}$ Auger production caused by the reduced portion of $\mathrm{H}, \mathrm{C}, \mathrm{N}$ and $\mathrm{O}$ elements and a lower fraction of Compton electrons, in favour of the increased fraction of $4 \mathrm{keV}$ Auger electrons from the calcium-rich rib tissue. As the $\mathrm{RBE}_{\mathrm{DSB}}$ is the smallest for rib (approximately 1.4) this would seem to indicate that the $\mathrm{RBE}_{\mathrm{DSB}}$ is more dependent on tissue composition (through the proportion of low energy $\mathrm{C}, \mathrm{N}$ and $\mathrm{O}$ Auger electrons) than on spectral hardening. 
Axxent: $40 \mathrm{kVp}$ Vs. $50 \mathrm{kVp}$
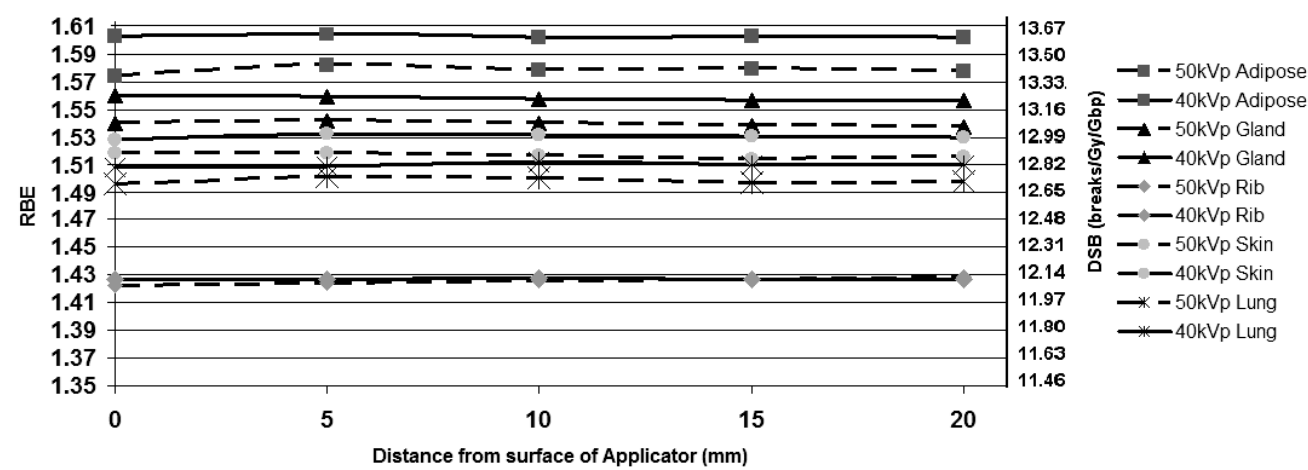

Figure 8: The DSB yield and $\mathrm{RBE}_{\mathrm{DSB}}$ for $40 \mathrm{kVp}$ and $50 \mathrm{kVp}$ Axxent eBt spectrum in tissue.

\section{RBE mapping of APBI patient}

A plot of $\mathrm{RBE}_{\mathrm{DSB}}$ vs. average photon energy is shown in Figure 9 for the two dominating tissue types in breast; gland and adipose. Similar relationships were calculated for other tissues with good correlation between average energy and $\mathrm{RBE}_{\mathrm{DSB}}$. Combination of the 40 and $50 \mathrm{kVp}$ results demonstrates a link between the average energy of a photon spectrum in these tissues and its RBE. This relationship was fit to a quadratic equation for each tissue, allowing a conversion factor for average photon energy to RBE. Figure 9 contains more points at greater distances $(0-40 \mathrm{~mm})$, allowing for a more detailed RBE vs. average energy relationship than seen in figure 6 and $8(0-20 \mathrm{~mm})$. 


\section{RBE vs. average photon energy}

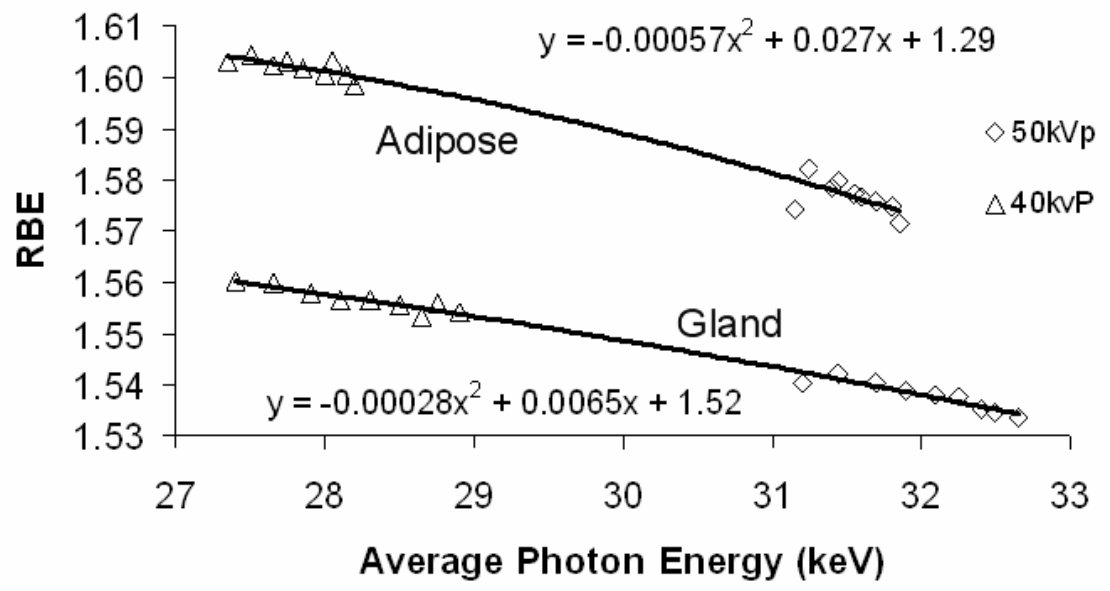

Figure 9: The $R B E_{D S B}$ vs. average photon energy plot for gland tissue $\left(R^{2}=0.99\right)$ and adipose $\left(R^{2}=0.99\right)$ for the Axxent eBt source

The mean photon energy map in an example breast case is shown in Figure 10. A RBE $E_{\mathrm{DSB}}$ map for the same case is shown using the $\mathrm{RBE}_{\mathrm{DSB}} /$ Average photon energy conversion equations from Figure 9. This figure shows the $\mathrm{RBE}_{\mathrm{DSB}}$ distribution in various tissues, as segmented in Figure 5. As had been observed in previous sections, the ribs show the lowest $\mathrm{RBE}_{\mathrm{DSB}}$ (approximately 1.4). The effect of spectral hardening on $\mathrm{RBE}_{\mathrm{DSB}}$ can be seen in adipose and gland tissues with a slight $(<2 \%)$ decrease from the centre of the balloon applicator. Skin demonstrated an $\mathrm{RBE}_{\mathrm{DSB}}$ between adipose and gland throughout, with only a slight decrease $(<2 \%)$ at the most distal regions. This kind of $\mathrm{RBE}_{\mathrm{DSB}}$ map may help to assess biological damage throughout breast tissue (or in any other applications of low energy brachytherapy sources).

In addition to the small changes in beam quality, other factors will likely play a more important role in RBE, such as the large fall-off in dose-rate at greater distances from the source. Consequentially the lack of a reduction in $\mathrm{RBE}_{\mathrm{DSB}}$ at large distances from the source may mean a greater degree of DNA damage in healthy tissue, however this is offset when taking into account the biological weighted dose (dose $\times$ RBE). The absence of any major impact of spectral hardening (Figure 9) on $\mathrm{RBE}_{\mathrm{DSB}}$ disagrees with the assertion of a number of studies (Brenner et al., 1999; Liu et al., 2013) that spectral hardening of the Intrabeam eBt source could cause a decrease in cell deactivation (and hence RBE) with distance from a source. 

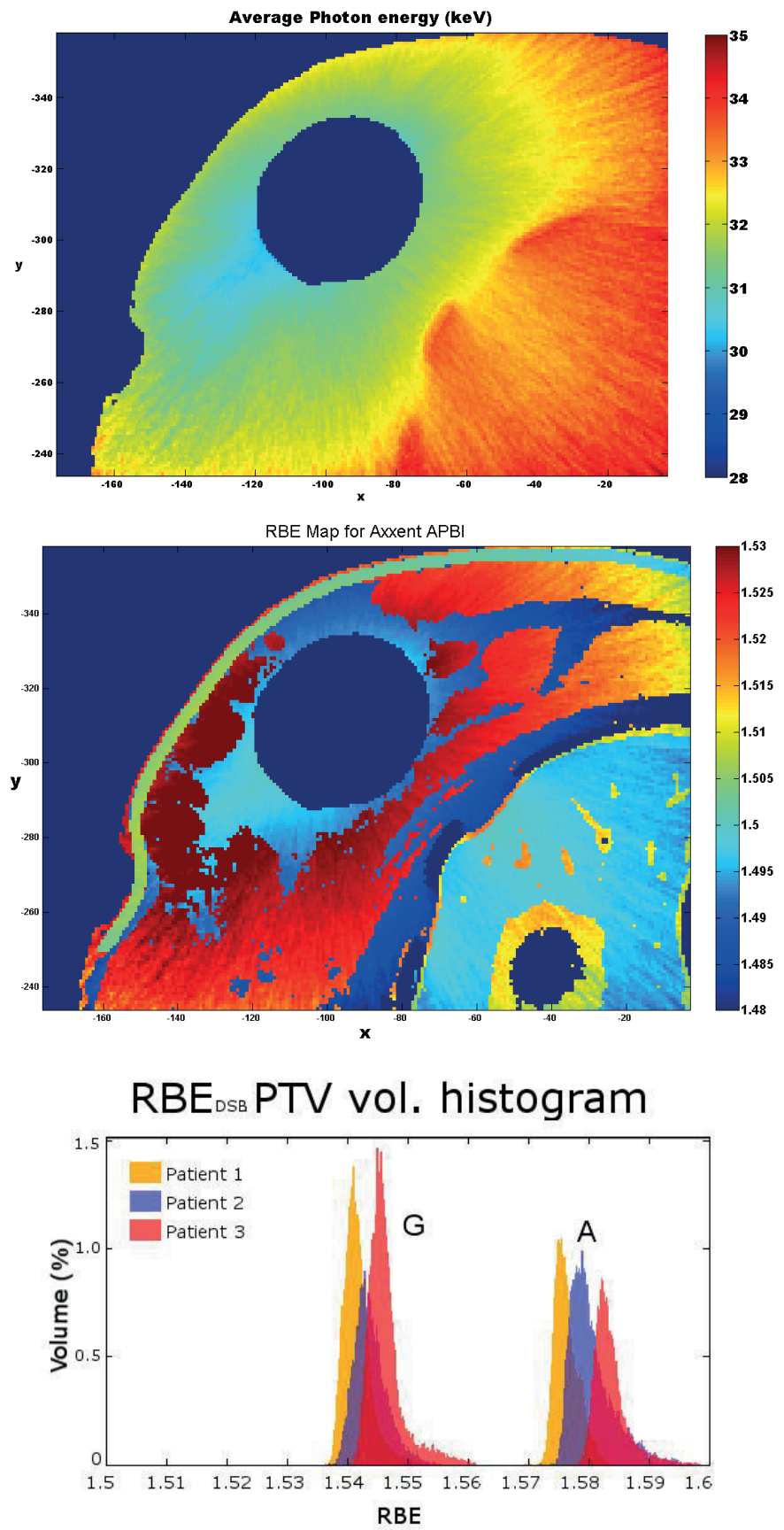

Figure 10: A single transverse slice in the source plane demonstrating an average photon energy map (top) and average $\mathrm{RBE}_{\mathrm{DSB}}$ map (center) of an APBI patient treated with the Axxent $50 \mathrm{kVp}$ source. A histogram of the RBE $_{\mathrm{DSB}}$ over the PTV volume for multiple patients (Bottom) shows the variation between adipose and gland. Peak amplitudes differ due to variations in the ratio of adipose/gland (Yaffe et al., 2009) and indicate a percentage of the PTV volume. $\mathrm{RBE}_{\mathrm{DSB}}$ in the balloon applicator and surrounding air were ignored. 


\section{Limitations}

Some assumptions about the composition of breast tissue and the composition and dimensions of the cell, inherent to the use of MCDS, are made. In this study, it is assumed that the ICRU tissue compositions in bulk tissue hold for the microscopic cellular level. However there are compositional differences between the cell components between cells which may result in a difference in electron spectra generated by cellular material and extracellular material particularly for short-range Auger electrons. The manual calculation of Auger electrons also holds some degree of uncertainty and serves only as an approximation of the actual Auger yield. The accuracy of the manual calculation is estimated to be about $20 \%$ by comparing Auger yields above $1 \mathrm{keV}$ for a uniform $\mathrm{NaCl}$ phantom with EGSnrc versus manual calculations; results not shown. The RBE impact of the Auger electrons is also uncertain. While Auger electrons have an influence on the RBE, the range of Auger electrons is small $(<0.1 \mu \mathrm{m})$ compared to an estimated human cell size $(10-100 \mu \mathrm{m}$ diameter range) so Auger electrons generated throughout the cell and DNA structure may have a greater impact on RBE than Auger electrons generated outside the cell (Thomson et al., 2013). Indeed, the cellular and DNA composition may be more important for determining the Auger yield from a photon spectrum than the average tissue composition. However as both the Intrabeam and Axxent eBt sources have comparable photo-electron spectra, it is unlikely that any inaccuracies in Auger electrons will result in differences in RBE between the two.

It should be noted that the percentage variation of RBE with distance is within the maximum accuracy of MCDS (1\%) compared to track-length DNA simulations(Hsiao and Stewart, 2008). The differences between each source and mode (Figure $6 \& 8$ ) relate to experimental results may be masked within the clinical uncertainty of referenced measured data $(\sim 7 \%)$ used to validate MCDS ( (Hsiao and Stewart, 2008).

\section{Conclusion}

The Axxent and Intrabeam eBt sources have small differences in their emitted photon spectra, resulting in insignificant differences $(<1 \%)$ in $\mathrm{RBE}_{\mathrm{DSB}}$ in all tissues investigated when calculated using MCDS. The contribution of low energy Auger electrons is important and therefore also proper modeling of the processes giving rise to them. The differences in $\mathrm{RBE}_{\mathrm{DSB}}$ are small for different $\mathrm{kVp}$ in the Axxent eBt source $(<1 \%)$ indicating that a lower $\mathrm{kVp}$ does not confer any significant advantage to RBE. Other factors such as dose-rate and cell repair time may be more important factors.

$\mathrm{RBE}_{\mathrm{DSB}}$ maps were used to visualize the changes in beam quality in a realistic patient geometry. The $\mathrm{RBE}_{\mathrm{DSB}}$ map highlights the dependence of $\mathrm{RBE}_{\mathrm{DSB}}$ on tissue composition. The $\mathrm{RBE}_{\mathrm{DSB}}$ map may be used, combined with a dose map, to allow biological weighted dose mapping. It may also be allowed to assess differences in biological damage in patients with large differences in gland/adipose tissue mixtures. 


\section{Acknowledgements}

This work is supported by Grant \#2011-700810 of the Canadian Cancer Society Research Institute (CCSRI). S.W. was funded by the MAASTRO-Atrium research grant and by Xoft Inc. BR was supported by a Marie Curie Reintegration Grant (Grant Agreement No. PIRG05GA-2009-247878 from FP7-PEOPLE-2009-RG). Xoft, Inc. is gratefully acknowledged for providing technical details for the simulations. Special thanks to Mark Podesta for his support with HTCondor, and to OpenLine for donating computation time, Randall Holt is thanked for providing the patient CT images. Extra special thanks to Gullermo Stutz for his contribution to Auger calculation, to Peter Watson for providing the Zeiss Intrabeam MC beam spectrum and to Dr Robert Stewart for useful discussions on MCDS.

\section{References}

Afsharpour H, Landry G, Reniers B, Pignol J P, Beaulieu L and Verhaegen F 2011 Tissue modeling schemes in low energy breast brachytherapy Phys Med Biol 56 7045-60

Agostinelli S, et al. 2003 Geant4: a simulation toolkit Nuclear Instruments and Methods in Physics Research Section A: Accelerators, Spectrometers, Detectors and Associated Equipment 506 250-303

Berger M J, Hubbell J H, Seltzer S M, Chang J, Coursey J S, Sukumar R and Zucker D S 2005 XCOM: Photon Cross Section Database (version 3.1). (Gaithersburg, MD: National Institute of Standards and Technology)

Bernal M A, deAlmeida C E, David M and Pires E 2011 Estimation of the RBE of mammography-quality beams using a combination of a Monte Carlo code with a B-DNA geometrical model Phys Med Biol 56 7393-403

Brenner D J and Amols H I 1989 Enhanced risk from low-energy screen--film mammography X rays $\mathrm{Br} J$ Radiol 62 910-4

Brenner D J, Leu C S, Beatty J F and Shefer R E 1999 Clinical relative biological effectiveness of low-energy xrays emitted by miniature $\mathrm{x}$-ray devices Phys Med Biol 44 323-33

Botchway S W, Stevens D L, Hill M A, Jenner T J and O'Neill P 1997 Induction and rejoining of DNA doublestrand breaks in Chinese hamster V79-4 cells irradiated with characteristic aluminum K and copper L ultrasoft X rays Radiat Res 148 317-24

Carlson D J, Stewart R D and Semenenko V A 2006 Effects of oxygen on intrinsic radiation sensitivity: A test of the relationship between aerobic and hypoxic linear-quadratic (LQ) model parameters Med Phys 33 $3105-15$

Cullen D H J, Kissel L. 1997 EPDL97: The Evaluated Photon Data Library, 97 version, LLNL Report No. UCRL-50400 Vol. 6

de Lara C M, Hill M A, Jenner T J, Papworth D and O'Neill P 2001 Dependence of the yield of DNA doublestrand breaks in Chinese hamster V79-4 cells on the photon energy of ultrasoft X rays Radiat Res 155 440-8

Deneve J L, Hoefer R A, Jr., Harris E E and Laronga C 2012 Accelerated partial breast irradiation: a review and description of an early North American surgical experience with the intrabeam delivery system Cancer Control 19 295-308

Eaton D J, et al. 2012 In vivo dosimetry for single-fraction targeted intraoperative radiotherapy (TARGIT) for breast cancer Int J Radiat Oncol Biol Phys 82 e819-24

Elliott R L, DeLand M, Head J F and Elliott M C 2011 Accelerated partial breast irradiation: initial experience with the Intrabeam System Surg Oncol 20 73-9

Friedland W, Jacob P, Paretzke H G, Merzagora M and Ottolenghi A 1999 Simulation of DNA fragment distributions after irradiation with photons Radiat Environ Biophys 38 39-47

Georgakilas A G, O'Neill P and Stewart R D 2013 Induction and repair of clustered DNA lesions: what do we know so far? Radiat Res 180 100-9 
Goodhead D T 1994 Initial events in the cellular effects of ionizing radiations: clustered damage in DNA Int J Radiat Biol 65 7-17

Grassberger C, Trofimov A, Lomax A and Paganetti H 2011 Variations in linear energy transfer within clinical proton therapy fields and the potential for biological treatment planning Int J Radiat Oncol Biol Phys $801559-66$

Hsiao Y and Stewart R D 2008 Monte Carlo simulation of DNA damage induction by x-rays and selected radioisotopes Phys Med Biol 53 233-44

ICRP 2007 The 2007 Recommendations of the International Commission on Radiological Protection. ICRP publication 103 Ann ICRP 37 1-332

International Commission on Radiation Units and Measurements 1992 Photon, Electron, Proton and Neutron Interaction Data for Body Tissues

Kawrakow I 2000 Accurate condensed history Monte Carlo simulation of electron transport. I. EGSnrc, the new EGS4 version Med Phys 27 485-98

Keisch M, Arthur D, Patel R, Rivard M and Vicini F 2007 American brachytherapy society breast brachytherapy task group

Kellerer A M 2002 Electron spectra and the RBE of X rays Radiat Res 158 13-22

Kirkby C, Ghasroddashti E, Poirier Y, Tambasco M and Stewart R D 2013 RBE of kV CBCT radiation determined by Monte Carlo DNA damage simulations Phys Med Biol 58 5693-704

Kraus-Tiefenbacher U, Scheda A, Steil V, Hermann B, Kehrer T, Bauer L, Melchert F and Wenz F 2005 Intraoperative radiotherapy (IORT) for breast cancer using the Intrabeam system Tumori 91 339-45

Kuhne M, Urban G, Frankenberg D and Lobrich M 2005 DNA double-strand break misrejoining after exposure of primary human fibroblasts to $\mathrm{CK}$ characteristic $\mathrm{X}$ rays, $29 \mathrm{kVp} \mathrm{X}$ rays and 60Co gamma rays Radiat Res $164669-76$

Liu D, Poon E, Bazalova M, Reniers B, Evans M, Rusch T and Verhaegen F 2008 Spectroscopic characterization of a novel electronic brachytherapy system Phys Med Biol 53 61-75

Liu Q, Schneider F, Ma L, Wenz F and Herskind C 2013 Relative Biologic Effectiveness (RBE) of 50 kV Xrays measured in a phantom for intraoperative tumor-bed irradiation Int J Radiat Oncol Biol Phys 85 $1127-33$

Maigne L, Perrot Y, Schaart D R, Donnarieix D and Breton V 2011 Comparison of GATE/GEANT4 with EGSnrc and MCNP for electron dose calculations at energies between $15 \mathrm{keV}$ and $20 \mathrm{MeV}$ Phys Med Biol 56 811-27

Mestres M, Caballin M R, Barrios L, Ribas M and Barquinero J F 2008 RBE of X rays of different energies: a cytogenetic evaluation by FISH Radiat Res 170 93-100

Nikjoo H, O'Neill P, Goodhead D T and Terrissol M 1997 Computational modelling of low-energy electroninduced DNA damage by early physical and chemical events Int J Radiat Biol 71 467-83

Nikjoo H, Bolton C E, Watanabe R, Terrissol M, O'Neill P and Goodhead D T 2002 Modelling of DNA damage induced by energetic electrons ( $100 \mathrm{eV}$ to $100 \mathrm{keV}$ ) Radiat Prot Dosimetry 99 77-80

Nikjoo H and Lindborg L 2010 RBE of low energy electrons and photons Phys Med Biol 55 R65-109

Perkins S C, DE. Chen, MH. Hubbell, JH. Rathkopf, JH. Scofield, J. 1997 Table and graphs of atomic subshell and relaxation data derived from the LLNL Evaluated Atomic Data Library (EADL) LLNL Report No. UCRL-50400 Vol. 30

Poon E and Verhaegen F 2005 Accuracy of the photon and electron physics in GEANT4 for radiotherapy applications Med Phys 32 1696-711

Poon E, Le Y, Williamson J F and Verhaegen F 2008 BrachyGUI: an adjunct to an accelerated Monte Carlo photon transport code for patient-specific brachytherapy dose calculations and analysis Journal of Physics: Conference Series 102012018

Rivard M J, Davis S D, DeWerd L A, Rusch T W and Axelrod S 2006 Calculated and measured brachytherapy dosimetry parameters in water for the Xoft Axxent X-Ray Source: an electronic brachytherapy source Med Phys 33 4020-32

Reniers B, Liu D, Rusch T and Verhaegen F 2008 Calculation of relative biological effectiveness of a lowenergy electronic brachytherapy source Phys Med Biol 53 7125-35

Reniers B and Verhaegen F 2006 The microdosimetry of low-energy photons in radiotherapy Radiat Prot Dosimetry 122 401-3 
Rivard M J, Davis S D, DeWerd L A, Rusch T W and Axelrod S 2006 Calculated and measured brachytherapy dosimetry parameters in water for the Xoft Axxent X-Ray Source: an electronic brachytherapy source Med Phys 33 4020-32

Sanders M E, Scroggins T, Ampil F L and Li B D 2007 Accelerated partial breast irradiation in early-stage breast cancer J Clin Oncol 25 996-1002

Segala J J, Cardarelli G A, Hiatt J R, Curran B H and Sternick E S 2011 Interface dosimetry for electronic brachytherapy intracavitary breast balloon applicators J Appl Clin Med Phys 123221

Semenenko V A and Stewart R D 2004 A fast Monte Carlo algorithm to simulate the spectrum of DNA damages formed by ionizing radiation Radiat Res 161 451-7

Stewart R D, Yu V K, Georgakilas A G, Koumenis C, Park J H and Carlson D J 2011 Effects of radiation quality and oxygen on clustered DNA lesions and cell death Radiat Res 176 587-602

Stutz G E 2014 Compton scattering cross section for inner-shell electrons in the relativistic impulse approximation Nuclear Instruments and Methods in Physics Research Section B: Beam Interactions with Materials and Atoms 319 8-16

Thomson R M, Tedgren A C and Williamson J F 2013 On the biological basis for competing macroscopic dose descriptors for kilovoltage dosimetry: cellular dosimetry for brachytherapy and diagnostic radiology Phys Med Biol 58 1123-50

Vaidya J S, et al. 2001 Targeted intra-operative radiotherapy (Targit): an innovative method of treatment for early breast cancer Ann Oncol 12 1075-80

Verhaegen F and Reniers B 2004 Microdosimetric analysis of various mammography spectra: lineal energy distributions and ionization cluster analysis Radiat Res $162592-9$

Watson P and Seuntjens J 2014 A monte carlo model of a miniature low-energy x-ray tube using EGSnrc Med Phys 41 24-24

Whitaker S J and McMillan T J 1992 Oxygen effect for DNA double-strand break induction determined by pulsed-field gel electrophoresis Int J Radiat Biol 61 29-41

White S A, Landry G, Fonseca G P, Holt R, Rusch T, Beaulieu L, Verhaegen F and Reniers B 2014 Comparison of TG-43 and TG-186 in breast irradiation using a low energy electronic brachytherapy source Med Phys 41061701

Yaffe M J, Boone J M, Packard N, Alonzo-Proulx O, Huang S Y, Peressotti C L, Al-Mayah A and Brock K 2009 The myth of the 50-50 breast Med Phys 36 5437-43 

Chapter 6: Discussion 
The challenges associated with accurate dose calculation, dosimetry and RBE modeling of low energy brachytherapy have been explored in this thesis. Aspects of the TG-186 recommendations were incorporated in dose calculations and how they compare to TG-43U1 were calculated and measured quantitatively. These included tissue heterogeneities, composition variability and applicator attenuation effects. While some radio-nuclides sources were analyzed in this thesis, the main focus of the thesis was mostly on electronic brachytherapy sources.

Chapters 2 and 3 have shown the influence of tissue composition variations, applicator dose perturbation and patient geometry considerations for the Axxent electronic brachytherapy source (EBS) compared to conventional water-based geometries. In chapter 3, potentially significant differences between TG-43 and TG-186 DVH metrics were calculated, particularly for the skin where patient-dependent peak dose decreases were observed. In chapter 2 , the dose uncertainty due to atomic trace composition was calculated. It was found that tissue uncertainties may reduce the $\mathrm{D}_{90}$ in permanent implanted prostate brachytherapy with I125 and Pd103 seeds by up to $15 \%$ when comparing simulated tissue with and without trace elements present. Both studies show the importance of tissue composition and accurately modeled patient geometries in low energy brachytherapy.

Several dosimetry methods for low energy HDR brachytherapy were explored in chapter 4: novel EPR alanine dosimeters, EBT2 radiochromic films and Monte Carlo simulations. The challenges associated with brachytherapy dosimetry, the steep dose gradients and finite source size, were highlighted as were the energy dependence and intrinsic efficiency of EBT2 film. While, ideally, detectors should exhibit no volume-averaging, self-attenuation, angular anisotropy or energy dependence, in practice these can only be minimized by the right selection of dosimeter on a source-by-source basis (Tanderup et al., 2013). Regardless of the suitability of EPR alanine or film dosimetry to HDR low energy brachytherapy, they may be unsuited to monitor time-resolved treatment delivery in patients or non-static phantoms due to position uncertainty or their inability to measure the instantaneous dose-rate. MOSFET, plastic scintillation or radioluminesce dosimeters have being proposed as candidates for high and low energy brachytherapy dose verification (Andersen et al., 2009; Cherpak et al., 2012; Kertzscher et al., 2011)

The RBE of two low energy electronic brachytherapy sources was explored in chapter 5, demonstrating the similarities in radiation quality between two sources with different spectral outputs. DNA damage simulations utilizing the spectral simulations of the Axxent electronic brachytherapy sources were combined with APBI patient CT datasets from chapter 3 to produce $\mathrm{RBE}_{\mathrm{DSB}}$ maps of whole breast to observe how spectral hardening affects radiation quality through a realistic patient geometry. The study challenges the notion that softer photon spectra always lead to higher beam qualities and provides a hypothesis for a 'plateau' in radiation response for bremsstrahlung sources for certain energy ranges and introduces the concept of RBE-weighted dose maps.

TG-186 has provided a framework for improvements to high and low energy brachytherapy, most notably recommending the use of model-based dose calculation algorithms. The chapters in this thesis aim to provide insight into the improvements possible in low energy brachytherapy using Monte Carlo-based calculation methods. The work described may also be used as a stepping stone to resolve numerous topics mentioned in TG-186. In chapter 3, 
describing APBI dose calculation, both $\mathrm{D}_{\mathrm{m}, \mathrm{m}}$ and $\mathrm{D}_{\mathrm{w}, \mathrm{m}}$ are reported for the PTV and skin OAR. Due to the fundamental quantitative differences between $\mathrm{D}_{\mathrm{m}, \mathrm{m}}$ and $\mathrm{D}_{\mathrm{w}, \mathrm{m}}$, it is unknown which dose reporting method best correlates to clinical outcome(Ma and $\mathrm{Li}, 2011 \mathrm{~b}$ ) (Tedgren and Carlsson, 2013). Indeed clinical studies which report $\mathrm{D}_{\mathrm{m}, \mathrm{m}}$ in brachytherapy are virtually non-existent. This provides an argument for the use of $\mathrm{D}_{\mathrm{w}, \mathrm{m}}$, in that all clinical experience has been using TG-43 $\left(\mathrm{D}_{\mathrm{w}, \mathrm{w}}\right)$, making $\mathrm{D}_{\mathrm{w}, \mathrm{m}}$ results consistent with reporting in previous studies(Beaulieu et al., 2012). Given the lack of experience with $\mathrm{D}_{\mathrm{m}, \mathrm{m}}$-based clinical studies, chapter 3 offers a useful methodology of correlating both $\mathrm{D}_{\mathrm{w}, \mathrm{m}}$ and $\mathrm{D}_{\mathrm{m}, \mathrm{m}}$ dose distributions with clinical outcome, whether it be survival or toxicity outcome, in prospective and retrospective studies.

The influence of backscatter on superficial OARs, in this case the skin, is also highlighted in chapter 3. As TG-43-based dose calculations consistently over-predict the dose to skin, MBCDA models which calculate backscatter correct could potentially permit dose escalation in patients with a lower calculated skin dose though it is unknown whether TG-43U1 skin dose tolerances, based on empirical clinical practice, are applicable. A previous study(Shi et al., 2010) into model-based dose calculation for APBI patients with the Axxent EBS predicted similar decreases $(17 \%)$ in maximum dose to the skin, however no attempt was made to correlate this to skin toxicity. Indeed limited skin toxicity data after APBI using EBS has been published. The only study for skin toxicity involving the Axxent source merely recommended an optimal method of balloon catheter insertion prior to irradiation in order to minimize theoretical skin dose(Hepel et al., 2010a) rather than correlate skin toxicity with dose in patients cohorts. As this study calculates skin dose based on current treatment planning system parameters (TG-43U1), it fails to account for non-patient backscatter considerations. Some skin-toxicity data is available for similar treatment modalities. One study has shown that dose to skin volume, in excess of $100 \%$ of prescription dose, has been correlated proportionally to severity and frequency of occurrence of skin toxicity for MammoSite-treated patients(Vargo et al., 2014b). As MammoSite uses the higher energy Ir192 source, dose distributions are not subject to tissue heterogeneities to the same degree as EBS.

Individual variability to tissue composition is a topic discussed in chapter 2. As had been shown in a previous study(Landry et al., 2010b) which demonstrated the sensitivity of dose calculations of low energy sources to population bulk tissue composition uncertainties/deviations in MBDCA, chapter 2 shows that the trace element composition also influences the dose distribution in a non-negligible way. A fully comprehensive H,C,N,O and trace composition of human tissues relevant to low energy sources is lacking. While certain studies provide population distributions of bulk tissue composition(White, 1986) and precise trace element composition (Carvalho et al., 2007b), combined studies could not be found. The differences in $\mathrm{H}, \mathrm{C}, \mathrm{N}$ and $\mathrm{O}$ concentration between cancerous and healthy tissue samples are also challenging as no bulk tissue composition data is known to exist for cancerous tissue. The increased trace element concentration for cancerous prostate tissue is not accounted for in the ICRU 46 report (International Commission on Radiation Units and Measurements, 1992), which only accounts for average healthy tissue composition. As seen in chapter 2, the impact of trace elements should not be understated and in the absence of more complete tissue composition data, trace elements, population/individual variability and the relative differences between healthy and cancerous tissue present a non-negligible 
uncertainty that should be included in model-based dose calculations. There may also be unknown elements to trace element composition in humans such as environmental factors specific to geographical regions/diet/genetics(Freeland-Graves et al., 2014). The eventual magnitude of this uncertainty merits future investigation.

Shortcomings and limitations in radiochromic film-based dosimetry were shown in chapter 5 while lithium formate EPR dosimeters were shown to be a more precise method of dose determination. The shortcomings for the EBT2 film may be due to the orientation and angle of irradiation with respect to the source. Dose gradients across the film did not allow for accurate dosimetry for points close to the source. While the need for accurate dosimetry in brachytherapy has been previously highlighted, the difficulties with these dosimeters may have a minimal impact on in-vivo dosimetry in APBI brachytherapy. Though film measurements at $10 \mathrm{~mm}$ presented with large uncertainties, in clinical situations, the inflatable Axxent balloon applicator surrounds the source, increasing source-to-tissue distance and reducing the steep dose gradient across the PTV, making EBS dosimetry close to the source $(<15 \mathrm{~mm})$ of lesser clinical importance.

EBT2 film also presents issues with regard to energy dependence, calibration and film heterogeneities that also exist for EBT3 film. A study of I-125 dosimetry using EBT3 film calibrated for low energy has shown demonstrated similar energy dependence when compared to film calibrated to 6MV photon(Morrison et al., 2014). As with chapter 5, the EBT3 film was calibrated for low energy sources using an orthovoltage external beam source and in addition to (separately) using $6 \mathrm{MV}, 200 \mathrm{kVp}$ and the I-125 brachytherapy source itself. Film calibrated using higher energy sources produced a poorer fit (and hence dose accuracy) for the low energy source while the $75 \mathrm{kVp}$ source generated the most accurate fit due to larger dose uncertainties inherent to brachytherapy.

The issues encountered with EBT2 film in chapter 5 may also be due to the relative position of the source with respect to the film plane during irradiation and the position during postirradiation scanning. In external beam radiotherapy film dosimetry, the film plane is typically perpendicular to the plane of the beam and sufficiently faraway from the active layer such that any regions of film off the central beam axis have a minimal angle of incidence (figure 1). As brachytherapy sources may be closer to the plane of the film, the angle of incidence increases. Post-irradiation scanning involves measurement of optical density of the film using light which, like EBRT, passes through the film plane perpendicularly. As the active layer of the film is not an infinitely thin detector, the mean distance radiation must transverse increases with decreasing source distance. This may be exasperated with larger film pieces at closer distances and inhibit film usage for aspects of brachytherapy such as anisotropy, F(r), measurements required to determine TG-43 parameters. 


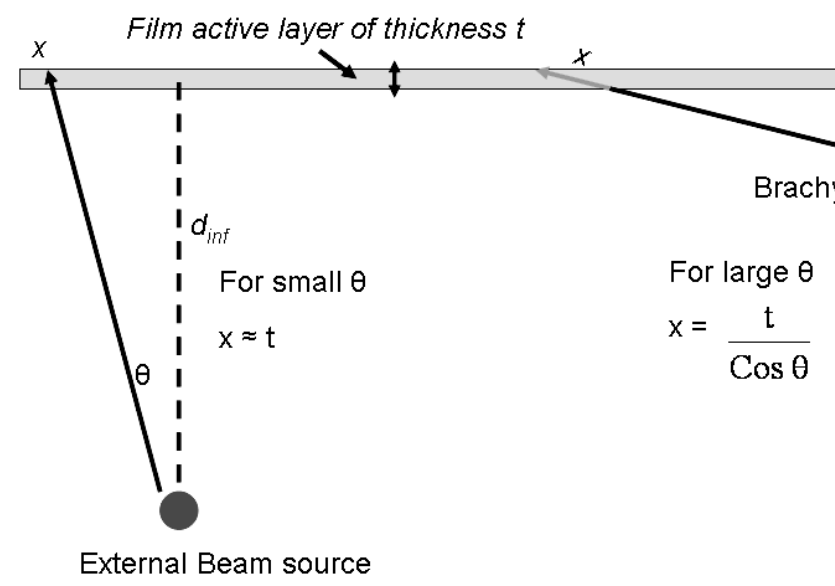

Figure 1: A schematic diagram representing photons passing through the film active layer (thickness t) from sources at different distances $d$. Photons traverses a length of $x$ through film which increases with increasing angle $\theta$.

It may be this factor that is responsible for the poor agreement seen in chapter 5 and further work is needed to determine if film orientation with respect to the source reduces accuracy. While the International Commission on Radiological Protection (ICRP, 2007)recommends assigning a radiation quality factor of unity for all photon emitting sources, previous studies (Reniers et al., 2008) have shown that $\mathrm{RBE}_{\mathrm{DSB}}$ for low energy brachytherapy sources is not unity. Work in Chapter 5 has shown similar values for $\mathrm{RBE}_{\mathrm{DSB}}$ (an indicator of radiation quality) for both EBS sources investigated and are in agreement with reported values in other studies(Liu et al., 2013; Marthinsen et al., 2010). As RBE is a complex quantity influenced by dose-rate, total dose and fractionation, the results reported in chapter 5 represent only one of the factors that determines biologically effectiveness. While both the Axxent and Intrabeam sources are used in the partial breast irradiation, the typical fractionation scheme and total dose for both sources are different (Herskind et al., 2005). The Intrabeam is used for single fraction IORT (Vaidya et al., 2001) while the Axxent may be used for single fraction IORT or for multi-fraction APBI(Dickler et al., 2009) Aspects of fractionation and dose delivery time have been explored in other studies (Herskind et al., 2005) and are beyond the scope of this thesis.

This thesis introduces $\mathrm{RBE}_{\mathrm{DSB}}$ maps for brachytherapy treatments. These provide a useful visual aspect to determining radiation quality throughout a realistic patient geometry, but it is not known how this element of RBE could be implemented clinically. Spectral hardening was not shown to significantly decrease $\mathrm{RBE}_{\mathrm{DSB}}$ throughout the breast geometry which is influenced more by tissue composition than by spectral changes. LET mapping in hadron therapy shows promise in identifying regions where particle slow-down, particularly around the Bragg peak, results in increased RBE locally, opening up the possibility of LET-weighted dose distributions(Grassberger et al., 2011). Ideally this would enable tighter dose-constraints for organs at risk. The degree to which $\mathrm{RBE}_{\mathrm{DSB}}$ mapping can be incorporated in future treatment planning systems is not yet known. While recommendations for the future of tissue and applicator heterogeneities, dose reporting and dosimetry in brachytherapy have been proposed (Beaulieu et al., 2012), no such recommendation have been given with respect to radiological modeling and $\mathrm{RBE}$. 


\section{References}

Andersen C E, Nielsen S K, Lindegaard J C and Tanderup K 2009 Time-resolved in vivo luminescence dosimetry for online error detection in pulsed dose-rate brachytherapy Med Phys 36 5033-43

Beaulieu L, et al. 2012 Report of the Task Group 186 on model-based dose calculation methods in brachytherapy beyond the TG-43 formalism: current status and recommendations for clinical implementation Med Phys 39 6208-36

Carvalho M L, Magalhaies T, Becker M and von Bohlen A 2007 Trace elements in human cancerous and healthy tissues: A comparative study by EDXRF, TXRF, synchrotron radiation and PIXE 9th Rio Symposium on Atomic Spectrometry 62 1004-11

Cherpak A J, Cygler J E, Andrusyk S, Pantarotto J, MacRae R and Perry G 2012 Clinical use of a novel in vivo 4D monitoring system for simultaneous patient motion and dose measurements Radiother Oncol 102 290-6

Dickler A, Ivanov O and Francescatti D 2009 Intraoperative radiation therapy in the treatment of early-stage breast cancer utilizing xoft axxent electronic brachytherapy World J Surg Oncol 724

Freeland-Graves J H, Sanjeevi N and Lee J J 2014 Global perspectives on trace element requirements $J$ Trace Elem Med Biol

Grassberger C, Trofimov A, Lomax A and Paganetti H 2011 Variations in linear energy transfer within clinical proton therapy fields and the potential for biological treatment planning Int J Radiat Oncol Biol Phys 80 1559-66

Hepel J T, Hiatt J R, Cardarelli G A and Wazer D E 2010 Modeling study for optimization of skin dose for partial breast irradiation using Xoft Axxent electronic brachytherapy applicator Brachytherapy 9 81-5

Herskind C, Steil V, Kraus-Tiefenbacher U and Wenz F 2005 Radiobiological aspects of intraoperative radiotherapy (IORT) with isotropic low-energy X rays for early-stage breast cancer Radiat Res $\mathbf{1 6 3}$ 208-15

ICRP 2007 The 2007 Recommendations of the International Commission on Radiological Protection. ICRP publication 103 Ann ICRP 37 1-332

International Commission on Radiation Units and Measurements 1992 Photon, Electron, Proton and Neutron Interaction Data for Body Tissues

Kertzscher G, Andersen C E, Siebert F-A, Nielsen S r K, Lindegaard J C and Tanderup K 2011 Identifying afterloading PDR and HDR brachytherapy errors using real-time fiber-coupled Al2O3:C dosimetry and a novel statistical error decision criterion Special Issue on Medical Physics and Brachytherapy 100 $456-62$

Landry G, Reniers B, Murrer L, Lutgens L, Gurp E B, Pignol J P, Keller B, Beaulieu L and Verhaegen F 2010 Sensitivity of low energy brachytherapy Monte Carlo dose calculations to uncertainties in human tissue composition Med Phys 37 5188-98

Liu Q, Schneider F, Ma L, Wenz F and Herskind C 2013 Relative Biologic Effectiveness (RBE) of $50 \mathrm{kV} \mathrm{X-}$ rays measured in a phantom for intraoperative tumor-bed irradiation Int J Radiat Oncol Biol Phys $\mathbf{8 5}$ $1127-33$

Ma C M and Li J 2011 Dose specification for radiation therapy: dose to water or dose to medium? Phys Med Biol 56 3073-89

Marthinsen A B, Gisetstad R, Danielsen S, Frengen J, Strickert T and Lundgren S 2010 Relative biological effectiveness of photon energies used in brachytherapy and intraoperative radiotherapy techniques for two breast cancer cell lines Acta Oncol 49 1261-8

Morrison H, Menon G and Sloboda R S 2014 Radiochromic film calibration for low-energy seed brachytherapy dose measurement Med Phys $\mathbf{4 1} 072101$

Reniers B, Liu D, Rusch T and Verhaegen F 2008 Calculation of relative biological effectiveness of a lowenergy electronic brachytherapy source Phys Med Biol 53 7125-35

Shi C, Guo B, Cheng C Y, Eng T and Papanikolaou N 2010 Applications of tissue heterogeneity corrections and biologically effective dose volume histograms in assessing the doses for accelerated partial breast irradiation using an electronic brachytherapy source Phys Med Biol 55 5283-97

Tanderup K, Beddar S, Andersen C E, Kertzscher G and Cygler J E 2013 In vivo dosimetry in brachytherapy Med Phys $\mathbf{4 0} 070902$ 
Tedgren A C and Carlsson G A 2013 Specification of absorbed dose to water using model-based dose calculation algorithms for treatment planning in brachytherapy Phys Med Biol 58 2561-79

Vaidya J S, et al. 2001 Targeted intra-operative radiotherapy (Targit): an innovative method of treatment for early breast cancer Ann Oncol 12 1075-80

Vargo J A, Verma V, Kim H, Kalash R, Heron D E, Johnson R and Beriwal S 2014 Extended (5-year) outcomes of accelerated partial breast irradiation using MammoSite balloon brachytherapy: patterns of failure, patient selection, and dosimetric correlates for late toxicity Int J Radiat Oncol Biol Phys $\mathbf{8 8} 285-91$

White H W a D 1986 The composition of body tissues Br. J. Radiol. 591209 

Chapter 7:

Valorization addendum 


\section{Innovation}

This thesis served to address the complexities inherent to dose calculation, dosimetry and radiobiological effectiveness with respect to electronic brachytherapy sources, in particular the Xoft Axxent.

\section{Model-based dose calculation algorithm}

The AAPM TG-56, which provides a code of practice for brachytherapy quality assurance, recommends a dose delivery accuracy of $5-10 \%$ to a target volume. The recommendations also state that dose distributions calculated using treatment planning software should be accurate to within $\pm 2 \%$. While certain dosimetry protocols (TG-43) assume that the patient can be assumed to be a water-sphere for dose calculation purposes, the complex patient composition and geometry present a challenge for low energy photon sources which are sensitive to composition variations and backscattering. The quantifying of these heterogeneities in chapter 2 and 3 can be used to improve dose delivery accuracy.

The combination of techniques for breast tissue segmentation and CAD-mesh applicator model allows more accurate assignment of heterogeneous tissues to patient geometries as well as modeling of sub-voxel size applicator features. A deformed inflatable balloon applicator would be difficult to model in Monte Carlo platforms using CSG.

\section{Relative biological effectiveness}

The low variability of radiobiological effectiveness in low energy brachytherapy for tissues calculated in chapter 5 demonstrate a lack of influence of beam hardening on radiation quality. This may reduce the complexity of low energy brachytherapy sources in terms of radiobiological considerations.

The similarity in RBE for electronic brachytherapy sources for different operating voltages $(40 \mathrm{kVp}$ and $50 \mathrm{kVp})$ seems to indicate that lower peak voltages does not confer a higher biological effectiveness.

\section{EPR Dosimetry}

The difficulties associated with in-vivo dosimetry around brachytherapy sources are wellestablished. Measurement of absorbed dose for low energy photons present extra challenges due to the material heterogeneities that enhance the dose differences between tissues more than at higher energies.

The results in chapter 4 show the viability of EPR Lithium-formate dosimetry at low energies due to the favourable dose response at distances close to the source. Lithium formate dosimeters are materially-equivalent to water and are sufficiently small, making them suitable for brachytherapy dosimetry. 


\section{Clinical Relevance}

The techniques for dose calculations and breast tissue segmentation described in chapter 3 can significantly improve dose calculation accuracy and may serve as a foundation for a Monte Carlo-based treatment planning system in the future. The lower-than-expected organat-risk dose in certain patients may permit dose escalation in certain patients that would be difficult to predict in TG-43-based planning systems.

Results describing the influence of trace elements on dose distributions for low energy sources can be incorporated into more accurately quantifying dose uncertainty. More accurate elemental tissue composition data may be obtained in the future and allow a further refinement of this uncertainty.

Chapter 5 describes predicts the iso-effectiveness between electronic brachytherapy, in terms of radiobiological effectiveness. While source-based RBE is not yet a criterion for photon radiotherapy (RBE assumed to be 1 for all sources), the various RBE described in tissue may be useful for incorporation into future treatment planning systems.

The clinical benefits of small brachytherapy dosimeters, described in chapter XX, are apparent given the difficulties in accurate dosimetry in low energy brachytherapy. Accurate pre-treatment planning and post-treatment evaluation using CT planning may not be possible, particularly in intra-operative radiotherapy using electronic brachytherapy sources. EPRbased dosimeters potentially offer greater re-usability and accuracy at shorter distances compared to EBT-based film.

\section{Societal relevance}

Brachytherapy is a radiotherapy treatment modality, widely used due to advantages it offers in treatment and cosmetic outcome. Brachytherapy treatment planning has not kept up with radiotherapy in terms of dose calculation and has only recently started to move away from water-based models.

The scientific findings discussed in this thesis can be used to improve dose calculation accuracy by properly accounting for applicator and tissue heterogeneities present, in particular, in breast patients. Improved accuracy may be useful for determining the possibility of dose escalation which can increase the dose to clinical relevant regions while maintaining an acceptable dose to organs at risk. The findings also serve to improve/refine dosimetric uncertainty in low energy brachytherapy in general.

\section{Commercial Relevance}

EPR dosimetry may become a standard quality assurance evaluator in low energy brachytherapy. The Monte Carlo techniques used may also be incorporated into a future Treatment planning system. 



\section{Acknowledgements}

First and foremost I would like to thank my supervisor, Frank, for supporting me until the end. It was a difficult 4 years and I certainly would not have made it if it were not for his help and guidance

Thanks also to Brigitte, both for her professional help and friendship throughout the four years.

In no particular order, I have to mention my colleagues who were good and loyal friends to me. Thank you, Stefan, Guillaume, Patrick, Skadi, Georgi, Isabel, Lotte, Ruben, Karen, Timo, Hoda, Ralph, Sara, Sean, Evelyn, Adrianna, Davide, Daan, Lucas, Wolter, Chin, Hoda, Ruud, Jackie-boy, Nessy and (because you were a smartass when I was writing these acknowledgements) last and least, JURGEN (Told ya)!

Special thanks to Mark, Gabriel and Aniek who have systematically tortured and abused me since 2013.

Apologies to Lillian A and Lillian B who had the insufferable job of cleaning my desk for 4 years.

Ruben, thanks for keeping me on time and not stashing the money away. Okay, Okay, thank you Skadi and Karen also.

Zuzie, Ro, Maria, Sarina, Sharo, Bubbles, Isa, Chantal and the Crossfitters, thanks for being my REAL, non-work friends ;).

Emelie, it was a nasty beast but we got it out there.

Finally, for his companionship on the lonely nights before submission, the only man I've ever loved, Bruce Springsteen.......... and Taylor Swift. 

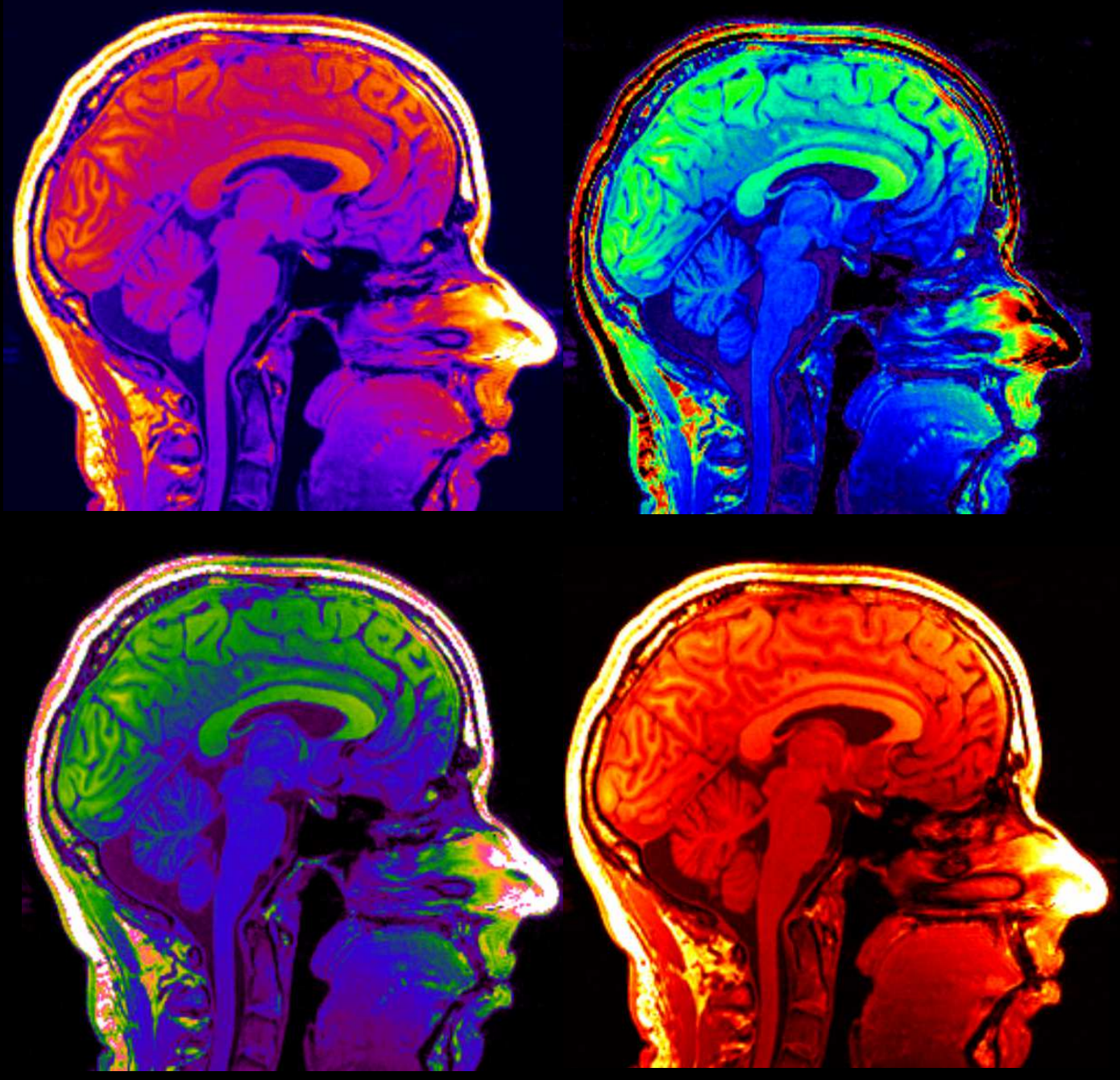